\title{
A cooler ion trap for the TITAN on-line trapping facility at TRIUMF
}

\author{
by \\ Zunjian Ke
}

\begin{abstract}
A Thesis
Submitted to the Faculty of Graduate Studies

in Partial Fulfillment of the Requirements

for the Degree of
\end{abstract}

Doctor of Philosophy

Department of Physics and Astronomy

University of Manitoba

Winnipeg, Manitoba

(C) Zunjian Ke, 2008 


\section{THE UNIVERSITY OF MANITOBA}

\section{FACULTY OF GRADUATE STUDIES \\ $\div * \div \div \div$}

\section{COPYRIGHT PERMISSION}

A Cooler Ion Trap for the TITAN On-Line Trapping Facility at TRIUMF

BY

\section{Zunjian Ke}

A Thesis/Practicum submitted to the Faculty of Graduate Studies of The University of Manitoba in partial fulfillment of the requirement of the degree

Of

Doctor of Philosophy

Zunjian Ke $\subset 2008$

Permission has been granted to the University of Manitoba Libraries to lend a copy of this thesis/practicum, to Library and Archives Canada (LAC) to lend a copy of this thesis/practicum, and to LAC's agent (UMI/ProQuest) to microfilm, sell copies and to publish an abstract of this thesis/practicum.

This reproduction or copy of this thesis has been made available by authority of the copyright owner solely for the purpose of private study and research, and may only be reproduced and copied as permitted by copyright laws or with express written authorization from the copyright owner. 


\begin{abstract}
The TITAN ion trap facility is currently being built at TRIUMF. For the first time, it will be possible to make precision mass measurements of highly charged radioactive ions, using the ISAC radioactive beam facility. Highly charged ions (HCI) extracted from the EBIT charge breeder will have a temperature of tens of $\mathrm{eV} /$ charge or more, too high for direct injection into the precision Penning trap mass spectrometer. For this reason, we are currently developing an intermediate Penning cooler trap to precool the $\mathrm{HCl}$ prior to the mass measurement. One possible route is electron cooling, where the HCI interact with a cloud of electrons, which self-cool via the emission of synchrotron radiation in the strong magnetic field of the cooler trap. Another possibility is sympathetic cooling with initially cold protons provided by a cold ion source. In this thesis, simulations of electron and proton cooling of highly charged ions are presented, including the potentially detrimental effects of radiative, dielectronic, and three-body recombination. Cooling times are given under different initial conditions for both proton cooling and electron cooling. A test geometry for the cooler trap is put into a computer simulation code such as SIMION, and injection of ions, electrons, and protons are simulated. Our preliminary design for a cooler trap which can accommodate both electron and proton cooling is presented.
\end{abstract}

Using singly charged ions, the mass excess of ${ }^{11}$ Be was measured at TITAN to be $20176.936(0.531) \mathrm{keV}$, which is about 10 times better than the previous value. In 
addition, an ion source was built for testing some of the TITAN subsystems ${ }^{1}$.

${ }^{1}$ This work was supported in part by grants from CFI and NSERC. 


\section{Acknowledgments}

I would like to express my gratitude to my supervisors Prof. G. Gwinner and Prof. W. T. H. van Oers, who gave me the chance to study for this degree in Canada. Thank you for your help during the period I worked on my thesis. Also, I would like to give my thanks to Prof. J. Dilling, who gave me the chance to join the TITAN project and gave me a lot of support. Thanks to Dr. D. Ramsay for many good suggestions about my thesis.

I would like to thank Dr. V. Ryjkov, Dr. R. Ringle, Dr. W.Shi, M. Brodeur, Dr. J. Vaz, Dr. M. Smith, Dr. Tao Sun, M.Good, L. Blomeley, D. Lindner, P. Grothkopp, A. Cefron and S.Toews; thank you for all your help while I worked on the TITAN project.

I thank Prof. K. Sharma for his useful discussions about the Penning trap, and I would also like to thank Dr. N. Oshima for the helpful discussions about the electron cooling calculations.

I would like to express my thanks to my G0 colleagues Dr. D. Ramsay, Dr. L. Lee and S. Chan. I learned a lot when I worked with you.

Finally, I would like to show my genuine thanks to my wife Xiaomei, my daughter Zimin and my parents for their unconditional support. 


\section{TABLE OF CONTENTS}

List of Figures $\quad$ iv

List of Tables $\quad$ x

Chapter 1: Introduction 1

1.1 Atomic mass models . . . . . . . . . . . . . . . . . . . . . . . 1

1.2 Weak interaction .................... 5

1.3 Astrophysical capture processes . . . . . . . . . . . . . . 8

1.4 Production of nuclei f. . . . . . . . . . . . . . . . . 8

1.5 Experimental methods of mass measurement . . . . . . . . . . . . 9

1.6 Outline of the thesis f . . . . . . . . . . . . . . 14

Chapter 2: $\quad$ The TITAN system at TRIUMF 15

2.1 Radio-frequency quadrupole . . . . . . . . . . . . . . 17

2.2 Electron beam ion trap . . . . . . . . . . . . . . 22

2.3 Penning trap . . . . . . . . . . . . . . . . . . . 28

2.3.1 Penning theory . . . . . . . . . . . . . . 28 


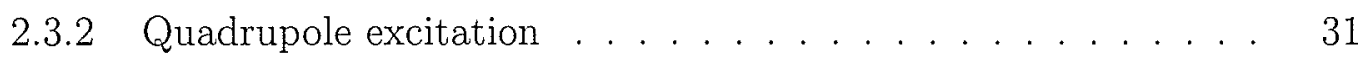

2.3.3 Time of flight . . . . . . . . . . . . . 34

2.4 Simulation for time of flight . . . . . . . . . . . . 35

2.5 Current status of TITAN . . . . . . . . . . . . 37

Chapter 3: $\quad$ Cooling techniques and electron-ion recombination $\quad 39$

3.1 Cooling methods . . . . . . . . . . . . . . 40

3.1.1 Resistive cooling ................. 40

3.1.2 Evaporative cooling .................. 41

3.1.3 Buffer gas cooling . . . . . . . . . . . . . . . 42

3.1 .4 Laser cooling ... . . . . . . . . . . . . . 45

3.1.5 Sympathetic cooling ................ 46

3.2 Electron-ion recombination ................. 58

$\begin{array}{lll}\text { Chapter 4: } & \text { Design of the cooler trap } & 66\end{array}$

4.1 Schematic diagram for cooling of HCIs . . . . . . . . . . . . . . 67

4.2 Designing potentials . . . . . . . . . . . . . . . 69

4.3 Injection of highly charged ions, electrons and protons . . . . . . . . . 74

4.3.1 Magnetic field . . . . . . . . . . . . . . . . 76

4.3.2 Simulation of charged particle injection . . . . . . . . . 77

4.4 Cooler trap implementation . . . . . . . . . . . . . . . . 89 
Chapter 5: Mass measurement of the halo nucleus beryllium-11 at $\begin{array}{ll}\text { TRIUMF } & 93\end{array}$

5.1 Measurement system of the TITAN Penning trap . . . . . . . . . 95

5.2 Analysis method . . . . . . . . . . . . . . . . . 98

$5.3{ }^{11}$ Be data analysis . . . . . . . . . . . . . . . 100

$\begin{array}{lll}\text { Chapter 6: } & \text { Summary and outlook } & 108\end{array}$

$\begin{array}{lll}\text { Chapter 7: } & \text { Appendix } & 111\end{array}$

7.1 Appendix A: Axial force depending on a magnetic gradient . . . . . . 111

7.2 Appendix B: Geometry file for simulation of time of flight . . . . . 113

7.3 Appendix C: User program for simulation of time of flight . . . . 115

7.4 Appendix D: User program for loading magnetic fields into SIMION . 123

7.5 Appendix E: Ion or proton injection geometry . . . . . . . . . 131

7.6 Appendix F: Ion source . . . . . . . . . . . . . . . 133

$\begin{array}{ll}\text { Bibliography } & 138\end{array}$ 


\section{LIST OF FIGURES}

1.1 Comparison of mass model predictions. . . . . . . . . . . . . . . 4

2.1 TITAN setup. . . . . . . . . . . . . . . . . . 16

2.2 RFQ electrode. . . . . . . . . . . . . . . . . . . . . . . . . 18

2.3 RFQ longitudinal potential. . . . . . . . . . . . . . . 19

$2.4 a-b$ stability region for the RFQ. . . . . . . . . . . . . . 22

2.5 RFQ built at TRIUMF. . . . . . . . . . . . . . . . 23

2.6 The principle of the EBIT. . . . . . . . . . . . . 24

2.7 Magnetic compression of an electron beam in a magnetic field. . . . . 27

2.8 The EBIT constructed at the Max-Planck-Institute for Nuclear Physics. 27

2.9 Hyperbolic Penning trap. . . . . . . . . . . . . . . . 29

2.10 Cylindrical Penning trap. . . . . . . . . . . . . . . . . . . . . 29

2.11 Hyperbolic Penning trap; the ring electrode is cut into four segments. $\quad 32$

2.12 Axial magnetic field $B_{z}$ at an off-axis distance of $0 \mathrm{~mm}$ and radial magnetic field $B_{r}$ for the precision Penning trap at an off-axis distance of $5 \mathrm{~mm} \ldots \ldots \ldots \ldots \ldots$ 
2.13 The system for time of flight. . . . . . . . . . . . . . 37

2.14 Time of flight from SIMION. . . . . . . . . . . . . . . . . 38

3.1 Energy evolution of various highly charged ions (HCI) during the proton-HCI cooling process. .......................... 48

3.2 Energy evolution of protons during the proton-HCI cooling process. 48

3.3 Energy dependence of $\mathrm{Kr}^{36+}$ on the ratio of the number of HCI $\left(N_{i}\right)$ to the number of protons $\left(N_{p}\right) \ldots \ldots \ldots 49$

3.4 Energy dependence of protons on the ratio of the number of HCI $\left(N_{i}\right)$ to the number of protons $\left(N_{p}\right)$. . . . . . . . . . . . . . . 49

3.5 Energy evolution of $\mathrm{Kr}^{36+}$ with different proton density and proton numbers during the proton- $\mathrm{Kr}^{36+}$ cooling process. . . . . . . . . . 50

3.6 Energy evolution of protons with different proton density and proton numbers during the proton- $\mathrm{Kr}^{36+}$ cooling process. . . . . . . . . 50

3.7 Energy evolution of $\mathrm{Kr}^{36+}$ with different initial $\mathrm{Kr}^{36+}$ energy during the proton-Kr${ }^{36+}$ cooling process. . . . . . . . . . . . . .

3.8 Energy evolution of protons with different initial $\mathrm{Kr}^{36+}$ energy during the proton- $\mathrm{Kr}^{36+}$ cooling process. . . . . . . . . . . .

3.9 Energy evolution of $\mathrm{Kr}^{36+}$ with different initial proton energy during the proton $-\mathrm{Kr}^{36+}$ cooling process. . . . . . . . . . . 
3.10 Energy evolution of protons with different initial proton energy during the proton- $\mathrm{Kr}^{36+}$ cooling process. . . . . . . . . . . . 52

3.11 Energy evolution of various highly charged ions (HCI) during the electron-HCI cooling process. ....................... 55

3.12 Energy evolution of electrons during the electron-HCI cooling process. 55

3.13 Energy dependence of $\mathrm{Kr}^{36+}$ on the ratio of the number of HCI $\left(N_{i}\right)$ to the number of electrons $\left(N_{e}\right) \ldots \ldots \ldots 6$

3.14 Energy dependence of electrons on the ratio of the number of $\mathrm{HCI}\left(N_{i}\right)$ to the number of electrons $\left(N_{e}\right) \ldots \ldots \ldots 6$

3.15 Energy evolution of $\mathrm{Kr}^{36+}$ with different initial $\mathrm{Kr}^{36+}$ energy during the electron $-\mathrm{Kr}^{36+}$ cooling process. . . . . . . . . . . 57

3.16 Energy evolution of electrons with different initial $\mathrm{Kr}^{36+}$ energy during the electron- $\mathrm{Kr}^{36+}$ cooling process. . . . . . . . . . . 57

3.17 Energy evolution of $\mathrm{Kr}^{36+}$ with different surrounding temperature during the electron- $\mathrm{Kr}^{36+}$ cooling process. . . . . . . . . . 58

3.18 Energy evolution of electrons with different surrounding temperature during the electron- $\mathrm{Kr}^{36+}$ cooling process. . . . . . . . . . . 58

3.19 Radiative recombination. . . . . . . . . . . . . . . . 59

$3.20 \mathrm{Fe}^{23+}$ radiative recombination coefficient. . . . . . . . . . . . 59

3.21 Dielectronic recombination. . . . . . . . . . . . . 60 
3.22 Three body recombination. . . . . . . . . . . . . . . . . 60

$3.23 \mathrm{Fe}^{23+}$ survival fraction at an environment temperature of $4 \mathrm{~K}$ including RR, DR, and TBR. . . . . . . . . . . . . . . 62

$3.24 \mathrm{Fe}^{23+}$ survival fraction at an environment temperature of $300 \mathrm{~K}$ including RR, DR, and TBR. . . . . . . . . . . . . . 62

$3.25 \mathrm{Fe}^{23+}$ survival fraction at an environment temperature of $4 \mathrm{~K}$ including

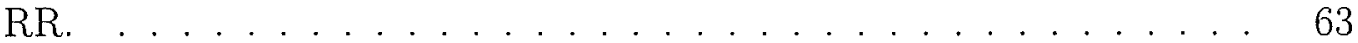

$3.26 \mathrm{Fe}^{23+}$ survival fraction at an environment temperature of $300 \mathrm{~K}$ including RR. . . . . . . . . . . . . . . . . . 63

3.27 Cooling of $\mathrm{U}^{92+}$ in the field-free case and cooling of $\mathrm{U}^{92+}$ with $\mathrm{B}=6 \mathrm{~T}$ from G. Zwicknagel. . . . . . . . . . . . . . . . . . . 65

4.1 Schematic diagram for proton cooling of HCIs. . . . . . . . . . . . . 67

4.2 Schematic diagram for electron cooling of HCIs. . . . . . . . . . . 68

4.3 Cylindrical trap. . . . . . . . . . . . . . . . . . 74

4.4 Cylindrical trap axial potential; the two curves are overlapped. . . . . 76

4.5 Proton injection, starting at a position $2400 \mathrm{~mm}$ from the center of the magnetic field. . . . . . . . . . . . . . . 80

4.6 Axial magnetic field curve for our 7 Tesla magnet. . . . . . . . . . . . 80

4.7 Enlarged trap region. . . . . . . . . . . . . . . . . 81 
4.8 Single proton injection with an initial proton position $4 \mathrm{~mm}$ from the central line. ....................... . . . 82

4.9 Single proton injection with an initial proton position $0 \mathrm{~mm}$ from the central line. . . . . . . . . . . . . . . . . . 82

4.10 Proton injection, starting at a position $1789 \mathrm{~mm}$ from the center of the magnetic field. . . . . . . . . . . . . . . . . . . 83

4.11 Proton injection, starting at a position $2600 \mathrm{~mm}$ from the center of the magnetic field. . . . . . . . . . . . . . . . . 84

4.12 Krypton injection. . . . . . . . . . . . . . 85

4.13 Single electron injection with an initial position $4 \mathrm{~mm}$ from the central line. ............................ 86

4.14 Electron injection. . . . . . . . . . . . . . . . 87

4.15 Electron synchrotron radiation. . . . . . . . . . . . . . 88

4.16 A schematic diagram for the cooler trap system. . . . . . . . . . . . 91

4.17 7 Tesla superconducting magnet made by Cryomagnetics, Inc. . . . . 92

5.1 The one neutron separation energy of Be versus A. . . . . . . . . 95

5.2 Injection system of the TITAN Penning trap. . . . . . . . . . . 96

5.3 Extracting system of the TITAN Penning trap. . . . . . . . . . 97

5.4 Structure of the Penning trap used at TITAN. . . . . . . . . . . . 97 
5.5 Penning trap axial potential. . . . . . . . . . . . . . . 102

5.6 EVA control. ...................... 103

5.7 Counts vs TOF for run 033066. . . . . . . . . . . . 103

5.8 Counts vs TOF for run 033072. . . . . . . . . . . . . 103

5.9 The spectrum of ion number per shot for run 033066. . . . . . . . . 104

5.10 The spectrum of ion number per shot for run 033072. . . . . . . . . . 104

5.11 TOF of reference ion ${ }^{6} \mathrm{Li}^{+} \ldots \ldots \ldots \ldots$

5.12 TOF of ion ${ }^{11} \mathrm{Be}^{+} \ldots \ldots \ldots \ldots \ldots$. . . . . . . . . . . 106

5.13 SOMA window for loading files. . . . . . . . . . . . . . . 107

5.14 SOMA window for results. . . . . . . . . . . . . . . 107

7.1 Ion source. . . . . . . . . . . . . . . . . . . . . . . . . . . . . 134

7.2 Emittance meter. . . . . . . . . . . . . . . . . . . . 135

7.3 Mechanical drawing of the emittance meter. . . . . . . . . . . . 136

7.4 Xe beam emittance and hydrogen beam emittance . . . . . . . . . 137 


\section{LIST OF TABLES}

3.1 Initial conditions for the energy evolution of various highly charged ions during the proton-HCI cooling process. . . . . . . . . . . . . . . 48

3.2 Initial conditions for the energy dependence of $\mathrm{Kr}^{36+}$ on the ratio of the number of HCI $\left(N_{i}\right)$ to the number of protons $\left(N_{p}\right) \ldots \ldots$

3.3 Initial conditions for the energy evolution of $\mathrm{Kr}^{36+}$ with different proton density and proton numbers during the proton $-\mathrm{Kr}^{36+}$ cooling process. $\quad 50$

3.4 Initial conditions for the energy evolution of $\mathrm{Kr}^{36+}$ with different initial $\mathrm{Kr}^{36+}$ energy during the proton $-\mathrm{Kr}^{36+}$ cooling process. . . . . . . .

3.5 Initial conditions for the energy evolution of $\mathrm{Kr}^{36+}$ with different initial proton energy during the proton $-\mathrm{Kr}^{36+}$ cooling process. . . . . . .

3.6 Initial conditions for the energy evolution of various highly charged ions during the electron-HCI cooling process. . . . . . . . . .

3.7 Initial conditions for the energy dependence of $\mathrm{Kr}^{36+}$ on the ratio of the number of HCI $\left(N_{i}\right)$ to the number of electrons $\left(N_{e}\right) \ldots \ldots$

3.8 Initial conditions for the energy evolution of $\mathrm{Kr}^{36+}$ with different initial $\mathrm{Kr}^{36+}$ energy during the electron- $\mathrm{Kr}^{36+}$ cooling process. . . . . . . 56 
3.9 Initial conditions for the energy evolution of $\mathrm{Kr}^{36+}$ with different surrounding temperature during the electron $-\mathrm{Kr}^{36+}$ cooling process. . . 57

3.10 Initial conditions for the $\mathrm{Fe}^{23+}$ survival fraction at a temperature of 4 K or $300 \mathrm{~K} \ldots \ldots \ldots \ldots 2$

4.1 Geometry of the cylindrical Penning trap and electrode voltages. . . .

4.2 $\mathrm{V}_{\text {calc }}$ : Voltages at the cylindrical electrodes from the analytical approach. $V_{S i m}$ : Voltages at the cylindrical electrodes found by our numerical method using SIMION. . . . . . . . . . . . . . . . . . . 75

5.1 The neutron separation energy of beryllium isotopes. . . . . . . . . . 94 


\section{Chapter 1}

\section{INTRODUCTION}

\subsection{Atomic mass models}

The atomic mass includes the interactions in the atom; it is not only the sum of the bare masses of its constituents but also a binding energy. A nuclide consists of protons, neutrons and electrons; the binding energy $B(N, Z)$ is

$$
B(N, Z)=\left[Z \cdot\left(m_{p}+m_{e}\right)+N \cdot m_{n}-M(Z, N)-B_{e} / c^{2}\right] \cdot c^{2}
$$

where the proton mass $m_{p}=938.272 \mathrm{MeV}$, the electron mass $m_{e}=0.511 \mathrm{MeV}$, the neutron mass $m_{n}=939.565 \mathrm{MeV}, Z$ is the number of protons, $N$ is the number of neutrons, $M(Z, N)$ is the atomic mass of a nuclide, and $B_{e}$ is the binding energy of the electrons. A detailed review on determination of nuclear masses was prepared by D. Lunney et al. [1]; the theory on mass models and experimental methods are described in it. The approaches to the formulation of mass models are roughly divided into macroscopic, microscopic and macroscopic-microscopic.

From a macroscopic aspect $[2,3,4]$, the binding energy simply depends on the number of nucleons. The well-known mass formula was given by von Weizsäcker in 
1935; subsequently it was improved by Bethe, Bacher, Myers and Swiatecki:

$$
B(N, Z)=a_{v o l} A-a_{s f} A^{2 / 3}-a_{c} Z^{2} A^{-1 / 3}-\left(a_{s y m} A+a_{s s} A^{2 / 3}\right) I^{2}
$$

where $I=(N-Z) / A, a_{v o l}, a_{s f}, a_{c}, a_{s y m}$, and $a_{s s}$ are the parameters of the volume term, the surface term, the Coulomb term, the asymmetry term, and the surfacesymmetry term, respectively.

Microscopic treatments involve the nonrelativistic Hartree-Fock approach or the relativistic Brueckner-Dirac method. A nonrelativistic Schrödinger equation can describe a nucleus in the nonrelativistic case if we know the interaction potential between proton and proton, proton and neutron etc. According to the Schrödinger equation

$$
H \psi=E \psi
$$

where the Hamiltonian $H$ can be given by

$$
H=-\frac{\hbar^{2}}{2 M} \sum_{i} \nabla_{i}^{2}+\sum_{i>j} V_{i j}+\sum_{i>j>k} V_{i j k} .
$$

If we can calculate the energy levels $E$, then the binding energy is simply given by $-E$. The interaction potential involves the two-nucleon interactions $V_{i j}$ and the three-nucleon interactions $V_{i j k}$. Strong, weak and electro-magnetic forces are involved in a nucleus; it is not a simple matter to find the proper potentials to describe the two-nucleon interactions and the three-nucleon interactions. The nonrelativistic Hartree-Fock approach uses an effective Hamiltonian to replace the real interaction 
Hamiltonian which is hard to define. The effective Hamiltonian is given by

$$
H_{e f f}=-\frac{\hbar^{2}}{2 M} \sum_{i} \nabla_{i}^{2}+\sum_{i>j} v_{i j}
$$

A suitable set of the ten-parameters $v_{i j}$ is given in references $[5,6,7]$ following the method of Vautherin and Brink. Furthermore, the like-nucleon pairing term and Wigner effect term should be included [8]. Between like nucleons, the $\delta$-function pairing term $[9,10]$ is given by

$$
v_{\text {pair }}\left(r_{i j}\right)=V_{\pi q} \delta\left(r_{i j}\right)
$$

The pairing-strength parameter $V_{\pi q}$ is different for neutrons and protons, and depends on whether $N$ is even or odd and whether $Z$ is even or odd. The Wigner effect term $[11,12]$ is another correction to binding energy. It can be given simply by

$$
E_{w}=V_{w} e^{(-\lambda|N-Z| / A)}
$$

When $\lambda \gg 1$, the extra energy exists primarily for $N=Z$. In the relativistic case, the proton and neutron are treated as Dirac spinors, and they can interact by the exchange of mesons [13].

Usually a combination of macroscopic and microscopic approaches is used. The binding energy is first calculated based on macroscopic methods, then shell effects, pairing, and Wigner effect etc. are taken into account.

Some mass formulas (see the details in reference [1]) such as the Skyrme-Hartree Fock mass formulas, the HFBCS-1, the HFB-1 mass formulas, the HFB-2 mass formula, the Duflo-Zuker mass formula, etc. are based on macroscopic, microscopic or 
macroscopic-microscopic models and use some independent mass-related parameters to fit a real data set consisting of thousands of masses. Different values of parameters, different effects, and different formulations of the same effect make results from different mass formulas very different, particularly for nuclei far from stability. Figure 1.1 shows the difference between the mass data and the predicted values for different mass formulas [14]. In the measured mass region, the difference is less than about 2 $\mathrm{MeV}$, while in the unstable mass region, there can be $10 \mathrm{MeV}$ mass difference between models. High precision mass measurement is very important to test these different mass models.

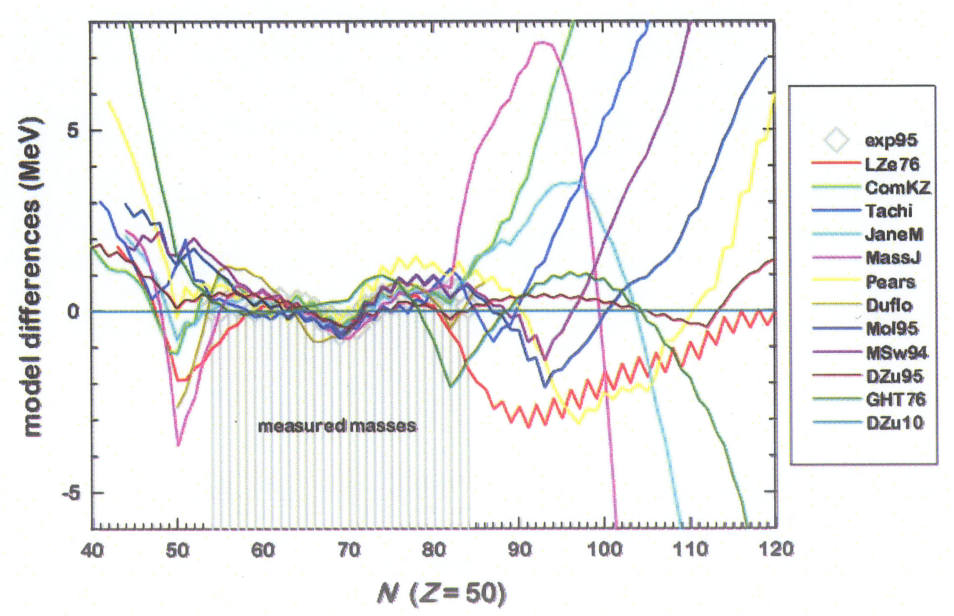

Figure 1.1: Comparison of mass model predictions. D. Lunney, http://www.nndc.bnl.gov/amdc/web/th-gen.html. 


\subsection{Weak interaction}

Another motivation for precision mass measurement comes from weak interaction studies. Quark eigenstates of the weak interaction are not the quark mass eigenstates of the strong interaction, but are related by the Cabbibo-Kobayashi-Maskawa $\operatorname{matrix}[15,16]$.

$$
\left(\begin{array}{c}
d_{w} \\
s_{w} \\
b_{w}
\end{array}\right)=\left(\begin{array}{ccc}
V_{u d} & V_{u s} & V_{u b} \\
V_{c d} & V_{c s} & V_{c b} \\
V_{t d} & V_{t s} & V_{t b}
\end{array}\right) \cdot\left(\begin{array}{c}
d_{s} \\
s_{s} \\
b_{s} .
\end{array}\right)
$$

Unitarity of the Cabbibo-Kobayashi-Maskawa matrix requires

$$
\left|V_{u d}\right|^{2}+\left|V_{u s}\right|^{2}+\left|V_{u b}\right|^{2}=1
$$

The probability of the quark transition $d \leftrightarrow u$ is given by $\left|V_{u d}\right|^{2}$.

The theory of $\beta$-decay was first developed by Fermi in 1934 [17]. The decay constant $\lambda$ is the probability per unit time of a transition between quantum states, and is given [18] by

$$
\lambda=\frac{2 \pi}{\hbar}\left|M_{f i}\right|^{2} \frac{d N}{d E_{T}}
$$

with

$$
M_{f i}=\int \Psi_{f}^{*} H \Psi_{i} d^{3} r
$$

where $H$ is the Hamiltonian operator associated with the weak interaction, $\frac{d N}{d E_{T}}$ is the density of available final states with the disintegration energy $E_{T}$, and $\Psi_{i}$ and $\Psi_{f}$ are 
the wave functions of the initial and final states. The half-life time is

$$
t_{1 / 2}=\frac{\ln 2}{\lambda}
$$

and the statistical rate function is given by

$$
f\left(Z, E_{T}\right)=\frac{1}{m_{e}^{5} c^{10}} \int_{0}^{E_{T}} F(Z, E) E\left(E^{2}-m_{e}^{2} c^{4}\right)^{1 / 2}\left(E_{T}-E\right)^{2} d E,
$$

A non-relativistic expression of the Fermi function is

$$
F(Z, E)=\frac{2 \pi \eta}{1-e^{-2 \pi \eta}}
$$

where $\eta= \pm Z e^{2} / \hbar v_{e}$, the plus sign is valid for the electron, the minus sign is valid for the positron, and $v_{e}$ is the electron velocity corresponding to electron energy E.

Generally, the $f t$-value which is the product of the statistical rate function $f$ and the half-life time $t$ is given $[19,20]$ by

$$
f t=\frac{K}{G_{V}^{2}\left|M_{F}\right|^{2}}
$$

where $K /(\hbar c)^{6}=2 \pi^{3} \hbar \ln 2 /\left(m_{e} c^{2}\right)^{5}=(8120.271 \pm 0.012) \times 10^{-10} \mathrm{GeV}^{-4} \mathrm{~s}, G_{V}$ is the vector coupling constant for semileptonic weak interactions, and $M_{F}$ is the Fermi matrix element. If the effective interaction is isospin invariant, the Fermi matrix element is given by

$$
\left|M_{F}\right|=<\Psi_{f}\left|T_{ \pm}\right| \Psi_{i}>=<T, T_{z} \pm 1\left|T_{ \pm}\right| T, T_{z}>=\sqrt{\left(T \mp T_{z}\right)\left(T \pm T_{z}+1\right)} .
$$

For superallowed Fermi $\beta$ decay between $T=1, J^{\pi}=0^{+}$states, where $J$ and $\pi$ designate the spin and parity of the initial (final) state, we can substitute $T=1$ 
and $T_{z}=1,0$, or -1 in Eq. 1.16, giving $\left|M_{F}\right|=\sqrt{2}$. Generally, from Eq. 1.11, the Hamiltonian operator $H$ and the $\Psi_{i}$ and $\Psi_{f}$ are very complicated [21]. Isospin is not an exact symmetry in nuclei, and $\left|M_{F}\right|^{2}=2\left(1-\delta_{C}\right)$, where $\delta_{C}$ is the isospin-symmetrybreaking correction which arises from the Coulomb and charge-dependent part of nuclear interaction Hamiltonian, the mixing among the $0^{+}$state wave functions which are connected with isospin in both the parent and daughter nuclei and small differences in the single particle neutron and proton radial wave functions. The emitted electron can emit a bremsstrahlung photon, in which case the statistical rate function $f$ will be replaced by $f\left(1+\delta_{R}\right)\left(1+\triangle_{R}^{V}\right)$, where $\delta_{R}$ and $\triangle_{R}^{V}$ represent respectively the nucleusdependent radiative correction and the nucleus-independent radiative correction. A "corrected" $f t$ value is

$$
\mathcal{F} t \equiv f t\left(1+\delta_{R}\right)\left(1-\delta_{C}\right)=\frac{K}{2 G_{V}^{2}\left(1+\triangle_{R}^{V}\right)}
$$

$G_{V}=\left|V_{u d}\right| G_{F} \mid[20,22]$, and $G_{F}$ is the Fermi coupling constant for purely leptonic decay.

The statistical rate function $f$ depends on the maximum energy of the electron, which in turn depends on the total transition energy $Q$ given by the mass measurement (masses of emitters and its daughters). By combining accurate mass measurements with half-life and branching ratio measurements, $V_{u d}$ can be determined and we can test the standard model. 


\subsection{Astrophysical capture processes}

Because of lack of data such as reaction cross sections, and the masses, etc. for exotic nuclei, it is hard to model astrophysical processes. It is believed that heavier elements form in stars via proton or neutron capture reactions, and knowledge of the proton or neutron separation energies can tell us how nucleosynthesis proceeds along the proton and neutron drip lines. The neutron drip line or the proton drip line is the line on the $Z, N$ plane where the neutron separation energy or the proton separation energy is zero. Mass measurements are very important to astrophysical capture processes $[23,24]$. The abundance can be calculated by

$$
\frac{Y_{n+1}}{Y_{n}}=\rho_{n} \frac{G_{n+1}}{2 G_{n}}\left(\frac{A_{n+1}}{A_{n}} \frac{2 \pi \hbar^{2}}{m_{u} k T}\right)^{3 / 2} \exp \left(\frac{S_{n+1}}{k T}\right)
$$

where $Y_{n}, Y_{n+1}, T, \rho_{n}, G, A, m_{\imath}, k, S$ are respectively the abundances of an initial and final nucleus of a single proton or neutron capture reaction in the chain, the temperature, the proton or neutron density, the partition function, the mass number, the atomic mass unit, the Boltzmann constant, and the proton or neutron separation energy. The proton or neutron separation energy depends on the mass difference of adjacent isotopes.

\subsection{Production of nuclei}

The Isotope Separator On-Line (ISOL) and in-flight methods can be used to produce different nuclei. The ISOL method [25] uses a high energy beam of light ions to 
bombard a thick target of heavier elements, and the reaction products from spallation, fission, and fragmentation processes are thermalized and transported by diffusion processes to an ion source for ionization and accelerated to a few tens of $\mathrm{keV}$. The nuclei are separated by electromagnetic fields. The ISOL method is used at ISOLDE at CERN and ISAC at TRIUMF. The in-flight method uses a heavy, high energy beam to bombard a thin target, and nuclides are produced through fusion-evaporation reactions. During the flight of the ions, the beam is separated by magnetic rigidity selection and a velocity filter, etc., and a gas stop cell is used to reduce the beam energy [26]. The in-flight method is used at GSI, ANL, RIKEN and the NSCL.

\subsection{Experimental methods of mass measurement}

The indirect method is to measure the $Q$-value. In a two-body reaction $a(b, c) d$, the mass excesses are related by $[27]$

$$
Q=\Delta_{a}+\Delta_{b}-\Delta_{c}-\Delta_{d},
$$

where $\Delta_{a}, \Delta_{b}, \Delta_{c}$, and $\Delta_{d}$ are the mass excesses for $a, b, c$, and $d$. If we know the mass excesses of three of $a, b, c$, and $d$, and measure the $Q$-value by measuring the kinetic energy, then the mass excess of the fourth one can be deduced. As an example of this

method, the only experimental value of the mass excess of ${ }^{11} \mathrm{Be}$ used for the AME2003 atomic mass evaluation was determined from the ${ }^{10} \mathrm{Be}(\mathrm{d}, \mathrm{p})^{11} \mathrm{Be}$ reaction [28].

The direct method uses the relation between the magnetic rigidity and the ion velocity or the cyclotron frequency to determine the mass of the ion; different methods 
are used at a few facilities.

Spectrometer SPEG at GANIL: Here, ion masses $(A \leq 70)$ are measured according to the relation

$$
B \rho=\gamma \frac{m v}{q}
$$

where $B \rho$ is the magnetic rigidity of the ion, $m, q, v$ and $\gamma$ are respectively the mass, charge, velocity, and the Lorentz factor. $v$ can be measured by time of flight [29] and once $B \rho$ is determined, the mass can be obtained from Eq. 1.20. A mass resolution of $2-4 \times 10^{-4}$ was reached at SPEG.

Schottky mass spectrometry at GSI: Here, ion mass measurements have been performed at the Experimental Storage Ring (ESR) [30]. The stored ions circulate in the ESR with characteristic revolution frequencies and induce a mirror charge on electrostatic pick-up electrodes. The unknown mass of an ion is determined by comparing to a known mass using

$$
\frac{f_{i}-f_{j}}{f_{i}}=-\alpha_{p} \cdot \frac{(m / q)_{i}-(m / q)_{j}}{(m / q)_{i}}+\left(1-\alpha_{p} \gamma^{2}\right) \frac{\Delta v}{v}
$$

where the revolution frequency $f_{i}$ is equal to $v_{i} / C_{i}, v_{i}$ and $C_{i}$ respectively are the velocity and length of the closed orbit, the momentum compaction factor $\alpha_{p}$ is equal to $d C / C /(d(B \rho) /(B \rho))$, and $B \rho$ is the magnetic rigidity; $f_{i}$ and $f_{j}$ are determined from broad-band Schottky spectra recorded by the pick-up electrodes. The ESR electron cooler provides a $\frac{\Delta v}{v}$ of $\sim 7 \times 10^{-7}$, and the second term in Eq. 1.21 is ignored. By comparing the results with reference ion measurements, the unknown 
mass can be determined with a relative precision of $10^{-6}[31]$.

Isochronous mass spectrometry at GSI: In Eq. 1.21, when $\alpha_{p} \gamma^{2}$ is equal to 1, it is the so-called isochronous mode. In this case, the second term is zero and electron cooling is not needed to reduce $\frac{\delta v}{v}$. A special thin-foil detection system records each passage of the stored ions, and the time of flight spectrum is analyzed by seeking a series of equidistant peaks. In this way, the revolution frequency $f=1 / T$ will be found, and by comparing with the time of flight for reference ions, the unknown mass may be determined with a relative precision of $2 \times 10^{-6}$ [32].

MISTRAL radio-frequency transmission spectrometer at CERN: The beam is injected into the homogeneous magnetic field region, where it follows a two-turn helical trajectory. At the end of the trajectory, the ions are recorded by a multiplier. Two radio-frequency electric field modulators are located at the one-half and three-half turn positions on the trajectory. Only when the modulation frequency $f_{R F}$ is equal to $(n+1 / 2) f_{c}\left(f_{c}\right.$ is the cyclotron frequency), the acceleration by one modulator will cancel the deceleration by the other, and the ions can go through the 0.4-mm narrow exit slit and be recorded by the multiplier. By making a wide modulation frequency scan, the cyclotron frequency can be found from the $f_{R F}$ of the peak position, and the mass of the ion can be determined to a relative precision of better than $1 \times 10^{-5}[33]$.

Penning trap: The radial energy of an ion in a Penning trap can be changed by applying a radio-frequency electric field at ring electrodes for a certain time period. When this radio-frequency is equal to the cyclotron frequency, the radial energy 
reaches a maximum. By measuring the time of flight after the ion is ejected from the Penning trap under a wide radio-frequency scan, the minimum time of flight will correspond to the maximum radial energy. So at the time of flight minimum, the radio-frequency is equal to the cyclotron frequency, and the mass of the ion can be determined (principle can be seen in Section 2.3).

Penning traps are used to measure the masses of isotopes; they exist at several facilities $[34,35]$, some of them are discussed in more detail below.

ISOLTRAP: Penning trap spectrometer: ISOLTRAP at ISOLDE/CERN was the first facility to use a Penning trap to measure the masses of short-lived radioactive ions. A radio-frequency quadrupole trap captures the continuous $60 \mathrm{keV}$ ISOLDE beam. Cooled and bunched ions are then re-accelerated to about $1 \mathrm{keV}$ and extracted into a buffer-gas filled preparation Penning trap. Isobar separation is done by using a mass selective buffer gas cooling technique [36] in which ions in the trap are excited by different radio-frequency fields. Finally, the ions are injected into a precision Penning trap for mass measurement. More than 200 masses have been measured by ISOLTRAP; an accuracy of typically $10^{-7}[37]$ can be reached.

CPT at Argonne National Laboratory: Here, ions are thermalized with helium gas in a gas catcher [38]. After passing through the RFQ ion guide region, bunched ions are extracted to an isotope separator, which is a cylindrical Penning trap, and then transfered to the Paul trap which is used for further ion cooling. Finally the ions are injected into the precision Penning trap $[39,26]$. Masses of isotopes such as 
${ }^{68} \mathrm{Ge},{ }^{68} \mathrm{Se}$ were measured by $\mathrm{CPT}$; these masses of waiting-point nuclides are very important to test models of the r-process. More than 60 radioactive isotopes with half-lives as short as one second have been measured with the CPT. The relative precision approaches $10^{-8}$.

LEBIT at NSCL at Michigan State University: A linear Paul trap system is used as the ion accumulator and buncher after ions first pass a very large gas stopping cell and RFQ ion-guide. Following the Paul trap system, the bunched beam of low energy and low emittance is injected into a Penning trap [40,41]. Masses of isotopes such as ${ }^{38} \mathrm{Ca},{ }^{65} \mathrm{Ge},{ }^{66} \mathrm{As},{ }^{67} \mathrm{As},{ }^{68} \mathrm{Se},{ }^{83} \mathrm{Kr},{ }^{84} \mathrm{Kr}$ were measured by LEBIT; a relative precision of about $10^{-8}$ can be reached $[42,43]$.

SMILETRAP at Stockholm: Because the relative precision depends on the charge of the ion, the relative precision of mass measurements can be improved by using highly charged ions. In SMILETRAP, stable highly charged ions are produced in the electron beam ion source CRYSIS in a similar way to the electron beam ion trap (details in Section 2.2), ions are injected into a pre-trap [44, 45]. The pre-trap is biased to CRYSIS potential, then, after ions are loaded, the biased voltage of the pre-trap is lowered to ground so that the energy of the highly charged ions can be reduced before the ions are injected into a precision Penning trap. The relative precision which can be reached is $1.5 \times 10^{-9}[46]$. 


\subsection{Outline of the thesis}

In my thesis, I shall first introduce the TITAN system and some subsystems in detail in Chapter 2; then I shall introduce the cooling techniques and electron-ion recombination in Chapter 3; I shall show a method to design the potential for the cooler trap, some results for the injection of protons, highly charged ion, and electrons, and a cooler trap implementation in Chapter 4; finally I shall show the result of mass measurements of the halo nucleus beryllium-11 at TRIUMF in Chapter 5. 


\section{Chapter 2}

\section{THE TITAN SYSTEM AT TRIUMF}

The ISAC (Isotope Separator and Accelerator) facility at TRIUMF is one of the world's most powerful radioactive beam facilities. It uses the $500 \mathrm{MeV}$, up to 100 $\mu \mathrm{A}$ proton beam which is produced by the TRIUMF cyclotron to bombard a thick target using the ISOL technique to produce radioactive isotopes $[47,48]$ in record quantities. The reaction products diffuse out of the hot target, and they are ionized and accelerated as a continuous beam. A dipolar magnet separator [49] is used to separate the ions; the typical resolving power is 3000 . The maximum beam energy is $60 \mathrm{keV}$ and the beam emittance is about $50 \pi \mathrm{mm}$ mrad. ISAC is one of the best places for an on-line mass measurement facility, and the TITAN project has been initiated for this purpose. TITAN encompasses new features not found at existing trap-based mass measurement setups; the most important one is the ability to use highly charged ions to carry out mass measurements. The mass measurement in a Penning trap is essentially the measurement of the cyclotron frequency in a known, constant magnetic field. Highly charged ions yield higher cyclotron frequencies and can increase the precision of the mass determination or alternatively, speed up the

measurement. The relative precision [50] in mass measurement using a Penning trap 
depends on the charge of the highly charged ion as shown by

$$
\frac{\delta m}{m} \approx \frac{m}{T_{r f} q B \sqrt{N}}
$$

where $N$ is the number of ions recorded, $T_{r f}$ is the quadrupolar excitation time, and $q$ is the charge of the ion. Making the mass measurement using highly charged ions can improve the precision of the mass measurement. TITAN is used to measure the mass of short-lived isotopes with half-lives of about $50 \mathrm{~ms}$, and a relative precision of $10^{-8}$ can be reached.

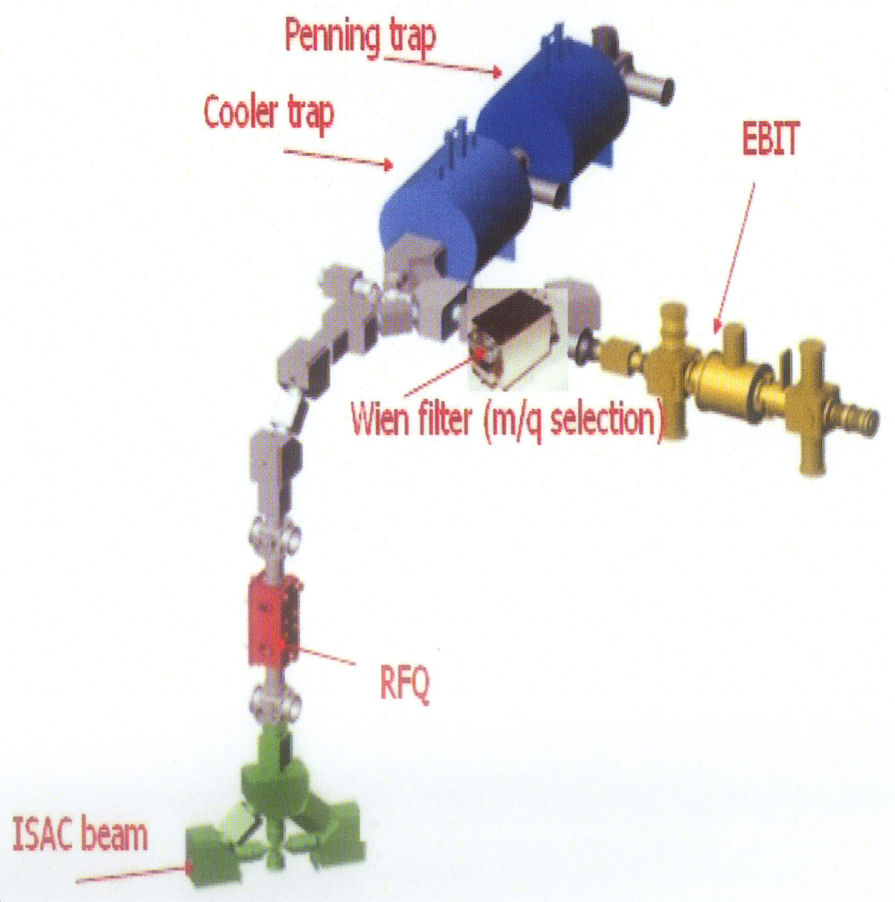

Figure 2.1: TITAN setup. 
Figure 2.1 shows the TITAN system: It consists of a radio-frequency quadrupole (RFQ), electron beam ion trap (EBIT), cooler trap, Wien filter, and Penning trap. The ISAC beam is a continuous beam. A radio-frequency quadrupole is used to cool and bunch the continuous ion beam, and the cooled and bunched ions are then injected into an EBIT. It breeds singly charged ions into highly charged ions within a few ms to tens of ms. Unfortunately, the HCI extracted from the EBIT will most likely have too large an energy spread for direct injection into the mass measurement trap. For this reason, we are developing a cooler trap in which the ion energy spread which is much more than $1 \mathrm{eV} / q$ can be reduced to about $1 \mathrm{eV} / q$. A Wien filter is used for selecting one charge state, because different charge states of ions exist when ions are extracted from the EBIT. Finally, the ions with low energy spread are injected into the precision Penning trap for precision mass measurement. In the following sections, the individual components of TITAN are introduced in more detail.

\subsection{Radio-frequency quadrupole}

The RFQ is used to reduce the emittance of the ISAC beam. Ions are trapped in the radial direction by applying radio-frequency signals at the cylindrical quadrants shown in Figure 2.2. Ions are trapped in the longitudinal direction by applying DC voltages at different electrodes along the longitudinal direction, resulting in the axial potential shown in Figure. 2.3. The RFQ is filled with helium buffer gas at a gas

pressure of about $2.5 \times 10^{-2}$ mbar. The buffer gas cools the ions (see the details about 
buffer gas cooling in section 3.1.3), which reduces the beam emittance. The cooling time is about $600 \mu s$. Finally, cooled ions are trapped in a small, deep potential region. By applying a kicking voltage at the last electrode of the RFQ, the trapped ions are ejected as a cold, bunched ion beam.

The RFQ [51] for TITAN is $700 \mathrm{~mm}$ long and consists of 24 segmented cylindrical quadrants with $r_{0}=10 \mathrm{~mm}$, where $r_{0}$ is the the minimum distance from the center of the RFQ to the cylindrical quadrants. Cylindrical quadrants of radius $1.148 r_{0}$ replace the ideal hyperbolic quadrants shown in the Figure 2.2.

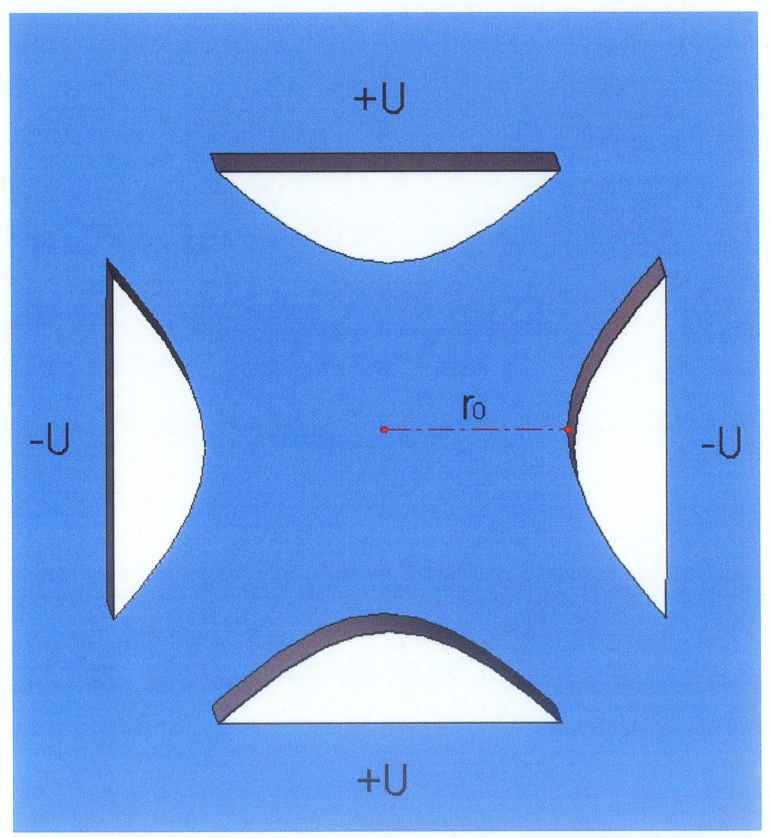

Figure 2.2: RFQ electrode.

Figure 2.2 shows a positive bias applied to one pair of electrodes and a negative 


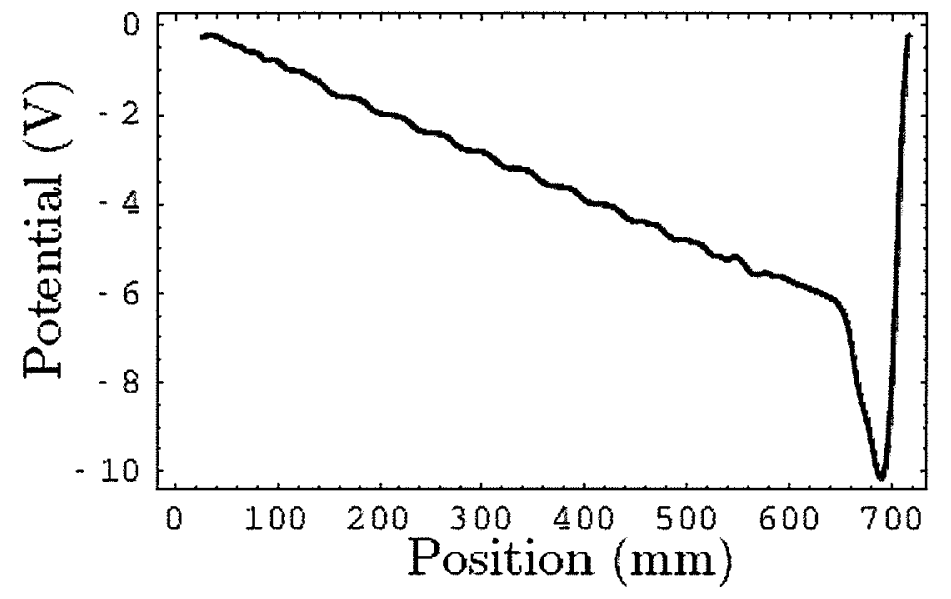

Figure 2.3: RFQ longitudinal potential. Courtesy of M. Smith, TITAN collaboration.

bias to the other pair,

$$
U=\frac{U_{0}-V_{0} \cos \omega t}{2} .
$$

The radial potential is

$$
\Phi(x, y)=\left[U_{0}-V_{0} \cos (\omega t)\right] \frac{\left(x^{2}-y^{2}\right)}{2 r_{0}^{2}},
$$

the radial electric field is

$$
\vec{E}=-\nabla \Phi(x, y)
$$

From Eq. 2.3 and Eq. 2.4, Newton's equation of radial motion for an ion with charge $q$ and mass $m$ is given by

$$
m \ddot{\vec{r}}=-q\left[U_{0}-V_{0} \cos (\omega t)\right] \frac{x \hat{x}-y \hat{y}}{r_{0}^{2}}
$$


with $\vec{r}=x \hat{x}+y \hat{y}$, Eq. 2.5 can be replaced by

$$
\ddot{x}=-\frac{q}{m} \frac{\left[U_{0}-V_{0} \cos (\omega t)\right]}{r_{0}^{2}} x
$$

and

$$
\ddot{y}=\frac{q}{m} \frac{\left[U_{0}-V_{0} \cos (\omega t)\right]}{r_{0}^{2}} y .
$$

By setting

$$
\begin{aligned}
& a=\frac{4}{\omega^{2} r_{0}^{2}}\left(\frac{q}{m}\right) U_{0} \\
& b=\frac{2}{\omega^{2} r_{0}^{2}}\left(\frac{q}{m}\right) V_{0}
\end{aligned}
$$

and

$$
\zeta=\frac{\omega t}{2}
$$

Eq. 2.6 and Eq. 2.7 become

$$
\frac{d^{2} x}{d^{2} \zeta}+(a-2 b \cos 2 \zeta) x=0
$$

and

$$
\frac{d^{2} y}{d^{2} \zeta}+(-a+2 b \cos 2 \zeta) y=0
$$

Eq. 2.11 and Eq. 2.12 have the form of a Mathieu equation. The general Mathieu equation (with parameters $a_{z}, q_{z}$ typically used in the literature) is

$$
\frac{d^{2} u}{d^{2} \zeta}+\left(a_{z}-2 q_{z} \cos 2 \zeta\right) u=0
$$

Solutions of Eq. 2.13 [52] have $a_{z}$ and $q_{z}$ which satisfy the relation 


$$
\beta=\sqrt{a_{z}+\frac{q_{z}^{2}}{2}}
$$

where $\beta$ has a value from 0 to 1 for practical RFQ implementations. When $\beta$ is varied in the range from 0 to 1 , then, for $x$ to be stable, according to the Eq. 2.14, $a$ and $b$ will be limited to a certain valid region (included by the two red dashed curves in Figure 2.4). For $y$ to be stable, $a$ and $b$ are constrained to another valid region (included by the two black curves). In the overlapping region shown in Figure 2.4 both $x$ and $y$ are stable, so if the frequency of the signal applied at the RFQ and its amplitude are selected correctly so that $a$ and $b$ fall in the overlapping region, then, ions can stably move in the RFQ; otherwise, they will be lost. When both $x$ and $y$ are stable, it means the ions are trapped in the radial direction. Because $a$ and $b$ depend on the mass of the ion, applying different frequencies of signals, certain kinds of ions can be trapped, while others are lost. The RFQ can therefore be used for mass separation.

Normally, ferrite core transformers are used to split the phase of sinusoidal RF signals which are applied to the electrodes of the RFQ. Because of the limited frequency range of such a system, a square-wave-driven system using digital circuitry is chosen for the RFQ at the TITAN facility. An amplitude of $400 V_{p p}$ at up to 1 $\mathrm{MHz}$ is reached by the square-wave-generator. Using a similar calculation method (see details in reference [51]) as above, for a square-wave-driven system, $b$, which is equal to $\frac{2}{\omega^{2} r_{0}^{2}}\left(\frac{q}{m}\right) V_{0}$, should be less than $0.712 ; \omega$ and $V_{0}$ are respectively the frequency 


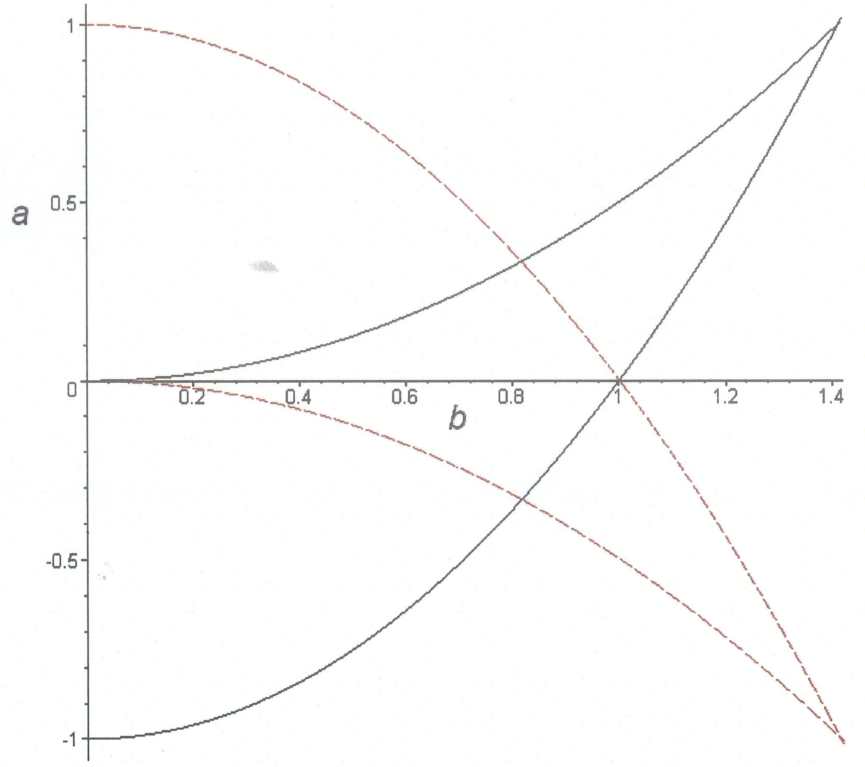

Figure 2.4: $a-b$ stability region for the RFQ.

and amplitude of the square-wave RF signal here. An emittance of $8 \pi \mathrm{mm}$ mrad for the extracted beam from the RFQ has been observed [53]; the typical extracted beam energy is $1 \mathrm{keV}$. The RFQ built at TRIUMF is shown in Figure 2.5.

\subsection{Electron beam ion trap}

The electron beam ion trap (EBIT) $[54,55,56]$ is a device used to create highly charged ions. It uses a combination of electrostatic fields and magnetic fields to confine the ions in three dimensions. Ions interact with the intense electron beam and can be stripped of one or more electrons by electron impact ionization. Highly charged ions can be produced by a stepwise ionization. Figure 2.6 shows the principles of an EBIT. 


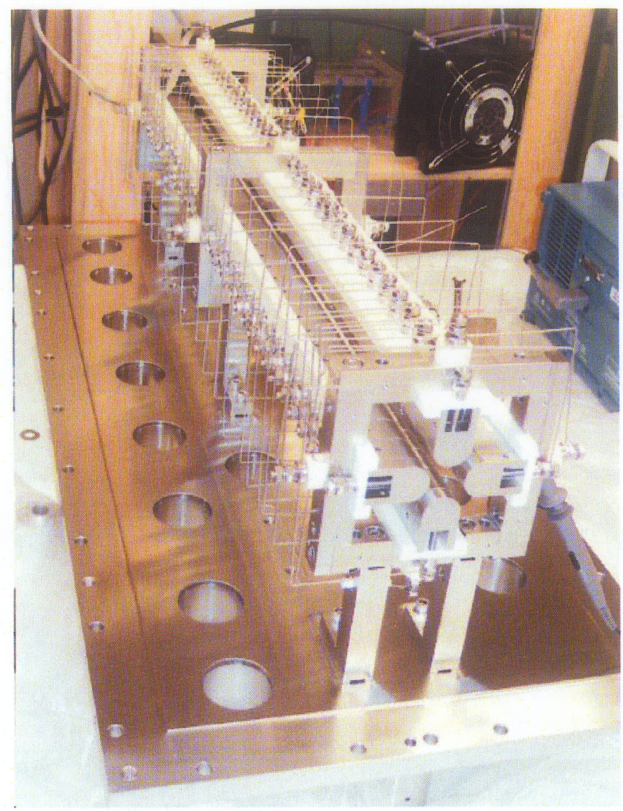

Figure 2.5: RFQ built at TRIUMF.

In the electron gun, the barium oxide cathode is heated to a high temperature $(\approx 1300$ K) and emits electrons. The focus electrode, anode, and negative floating voltage guide the electrons to the central trap region. The electrons follow the magnetic field lines. By placing the electron gun in a weak magnetic field region and the trapping region in a high field location, the electron beam is radially strongly compressed. Trim coils and bucking coils create a compensating magnetic field at the electron gun, which cancels the residual magnetic field generated by the Helmholtz coils (two identical circular magnetic coils which are placed symmetrically on two sides along the axis and separated by a distance which is equal to the radius of the coil) which supply the homogeneous magnetic field for the EBIT central trap region. The radial 


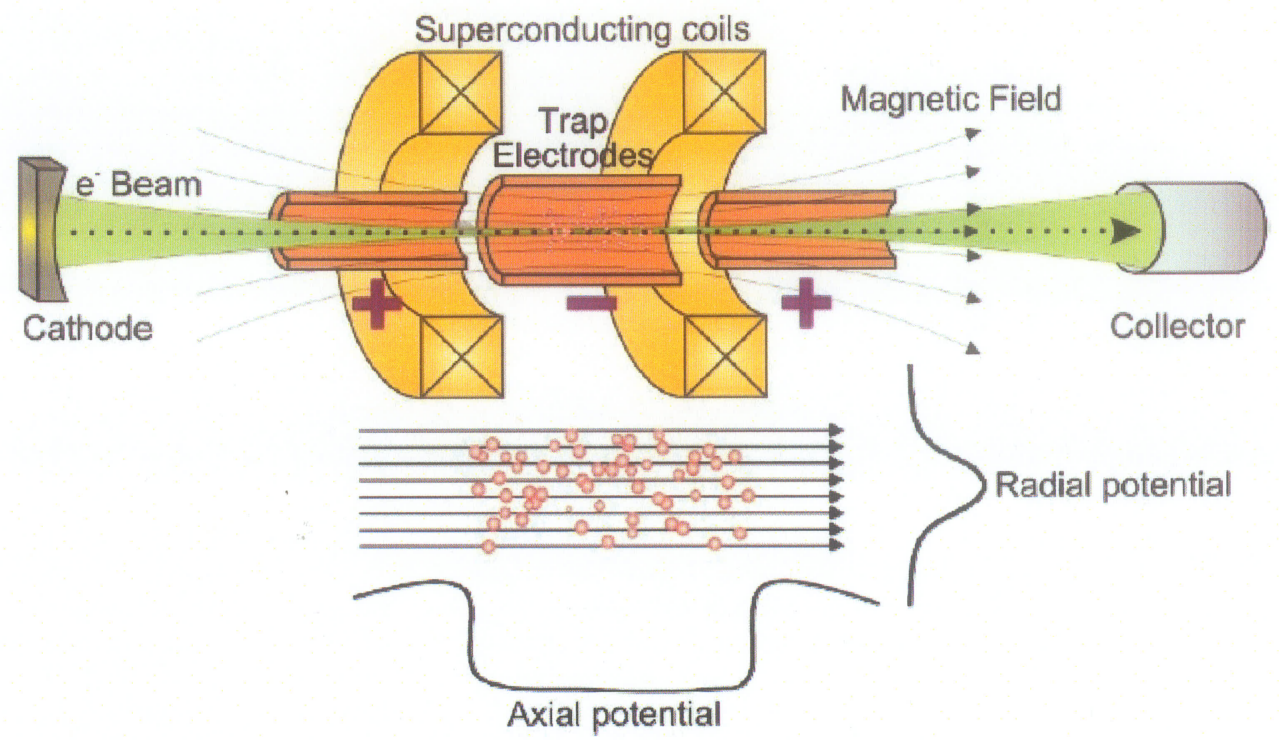

Figure 2.6: The principle of the EBIT. Courtesy of Hjalmar Bruhns.

electron distribution is Gaussian, and the radius (80\% electrons contained) [57] is given by

$$
r_{e}=r_{B}\left\{\frac{1}{2}+\frac{1}{2}\left[1+4\left(\frac{8 m k T_{c} r_{c}^{2}}{e^{2} B^{2} r_{B}^{4}}+\frac{B_{c}^{2} r_{c}^{4}}{B^{2} r_{B}^{4}}\right)\right]^{1 / 2}\right\}^{1 / 2}
$$

where $r_{B}$ is the Brillouin radius, given by

$$
r_{B}=\left(\frac{2 m^{3}}{\pi^{2} \varepsilon_{0}^{2} e^{2}}\right)^{1 / 4} \frac{I_{e}^{1 / 2}}{B E_{e}^{1 / 4}},
$$

where $m$ is the electron mass, $e$ is the electron charge, $B$ is the axial homogeneous magnetic field, $B_{c}$ is the magnetic field at the cathode, $r_{c}$ and $T_{c}$ are respectively the radius and temperature of the cathode, $I_{e}$ is the electron beam current, and $E_{e}$ is the electron energy. The electrons are finally collected by the collector. 
The processes which control the ion number balance in the EBIT are $(i)$ electronimpact ionization, $(i i)$ radiative recombination, $(i i i)$ charge exchange, and (iv) radial and axial ion escape from the trap. The differential equation [58] of the density $n_{i}$ for charge states $i$ is given by

$$
\begin{aligned}
\frac{d n_{i}}{d t}= & n_{e} v_{e}\left[\sigma_{i-1 \rightarrow i}^{i o n} n_{i-1}-\left(\sigma_{i \rightarrow i+1}^{i o n}+\sigma_{i \rightarrow i-1}^{R R}\right) n_{i}+\sigma_{i+1 \rightarrow i}^{R R} n_{i+1}\right] \\
& -n_{0} v_{i o n}\left[\sigma_{i \rightarrow i-1}^{c h e x} n_{i}-\sigma_{i+1 \rightarrow i}^{\text {chex }} n_{i+1}\right]-\nu_{i}^{\text {coll }} \frac{\exp \left\{-\frac{i e U_{w}}{k T_{i o n}}\right\}}{\frac{i e U_{w}}{k T_{i o n}}} n_{i}
\end{aligned}
$$

where $n_{e}$ is the density of the electrons, $v_{e}$ is the velocity of the electron, $n_{0}$ is the neutral gas density, $v_{i o n}$ is the average thermal ion velocity, $\sigma$ is the cross-section for different reaction processes (impact ionization, radiative recombination, and charge exchange), $\nu_{i}^{\text {coll }}$ is the collision rate for all Coulomb collisions of ions which have charge $i, U_{w}$ is the depth of the electrostatic potential well, and $k T_{i o n}$ is the thermal energy of the Coulomb-heated ions.

The electron-impact ionization process is

$$
A^{q+}+e^{-} \rightarrow A^{(q+1)+}+e^{-}+e^{-}
$$

The ion loses an electron and the charge state increases by 1 . The electron-impact ionization cross-section $[59,60,61]$ is given by

$$
\sigma_{i \rightarrow i+1}=\sum_{j=1} \frac{a_{i j} q_{i j}}{E_{e} P_{i j}} \ln \frac{E_{e}}{P_{i j}}\left\{1-b_{i j} \exp \left[-c_{i j}\left(E_{e} / P_{i j}-1\right)\right]\right\}
$$

where $j$ is summed over all $n l$ subshells, $E_{e}$ is the impact electron energy, $P_{i j}$ is the ionization energy of the $j$ th $n l$ subshell, $q_{i j}$ is the number of electrons in the $j$ th $n l$ subshell, and $a_{i j}, b_{i j}, c_{i j}$ are parameters. 
The charge exchange process arises from the interaction of ions and neutral gas. The reaction process for one-electron transfer is

$$
A^{q+}+B \rightarrow A^{(q-1)+}+B^{1+}
$$

The charge exchange cross-section [62] (in $\mathrm{cm}^{2}$ at collision energy below $25 \mathrm{keV} / \mathrm{amu}$ ) is given by

$$
\sigma_{i \rightarrow i-1}=1.43 \times 10^{-12} Z_{i}^{1.17} P_{0}^{-2.76}
$$

where $Z_{i}$ is the ion charge number, $P_{0}$ is the ionization potential (in $\mathrm{eV}$ ) of the neutral gas. The charge exchange process for the multiple electron transfer is

$$
A^{q+}+B \rightarrow A^{(q-r)+}+B^{r+} \rightarrow A^{(q-p)+}+B^{r+}+(r-p) e^{-},
$$

where $r$ is the number of electrons used for charge exchange, and $p$ is the number of electrons retained by the ion. The charge exchange cross-section [63] (in $\mathrm{cm}^{2}$ ) for multiple electron transfer can be approximated for slow collisions (this expression is valid for collision velocities below roughly 0.2 a.u.) as

$$
\sigma_{i \rightarrow i-r}=\left(2.7 \times 10^{-13}\right) Z_{i} r /\left[I_{1}^{2} I_{r}^{2} \sum_{j=1}^{N}\left(j / I_{j}^{2}\right)\right]
$$

where the ionization potential $I$ is in units of $\mathrm{eV}$, and $N$ is the number of outer-shell electrons.

Figure 2.7 shows how a $25 \mathrm{keV}$ electron beam moving from the right to the left (from low to high magnetic field) can be compressed by the strong magnetic field (SIMION simulation results, using the magnetic field which is shown in Figure 2.12). 
Figure 2.7: Magnetic compression of an electron beam in a magnetic field (spacecharge effects are not included).

The TITAN electron beam ion trap was designed and constructed at the MaxPlanck-Institute for Nuclear Physics (MSc thesis, M. Froese, University of Manitoba, 2006 [64]). The design electron current is $5 \mathrm{~A}$, the electron beam energy is $60 \mathrm{keV}$, and most ions can be bred into He-like ions with a charge breeding time of tens of ms. Using $27 \mathrm{keV}$, up to $500 \mathrm{~mA}$ electron beam, charge states as high as $\mathrm{Kr}^{34+}$ and $\mathrm{Ba}^{54+}$ have been reached for TITAN EBIT, which is shown in Figure 2.8.

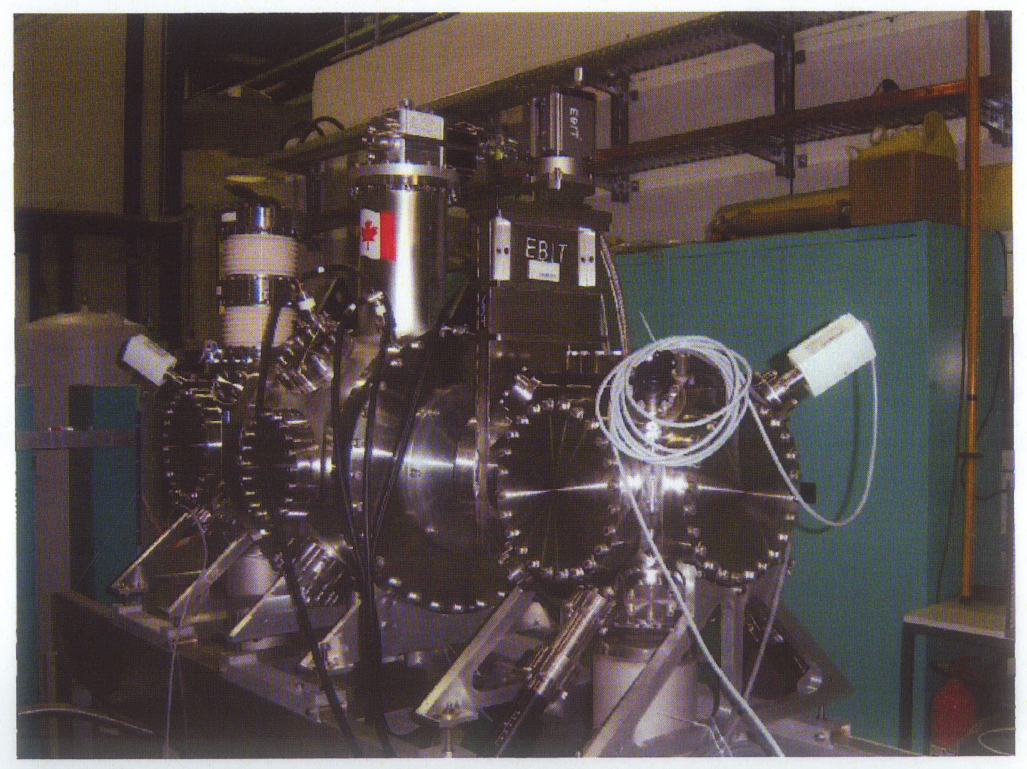

Figure 2.8: The EBIT constructed at the Max-Planck-Institute for Nuclear Physics. 


\section{$2.3 \quad$ Penning trap}

Penning traps have become very accurate tools for mass measurements both on stable and unstable isotopes. The Penning trap is a device that traps charged particles by using static electric and magnetic fields $[65,36]$.

\subsubsection{Penning theory}

A hyperbolic Penning trap is shown in Figure 2.9. It consists of a ring electrode and two endcap electrodes. The ring electrode is created by the hyperbola of revolu$\operatorname{tion}[65]$

$$
z^{2}=\frac{1}{2}\left(x^{2}+y^{2}-r_{0}^{2}\right)
$$

and the two endcap electrodes are created by the branches of the hyperbola of revolution

$$
z^{2}=z_{0}^{2}+\frac{x^{2}+y^{2}}{2}
$$

where $r_{0}$ is the minimum radial distance from the center of the trap to the ring electrode, and $z_{0}$ is the minimum axial distance from the center of the trap to the endcap electrodes.

The cylindrical Penning trap shown in Figure 2.10 consists of the cylindrical ring electrode, cylindrical compensation electrodes and cylindrical endcap electrodes.

The hyperbolic potential in Figure 2.9 has the form

$$
U(x, y, z)=\frac{U_{0}\left(2 z^{2}-x^{2}-y^{2}\right)}{4 d^{2}}
$$




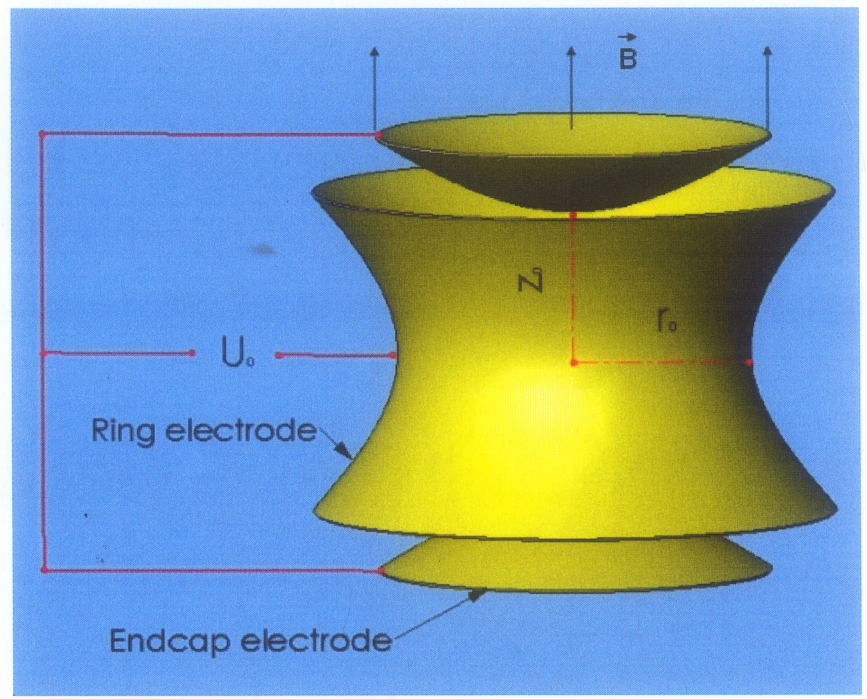

Figure 2.9: Hyperbolic Penning trap.

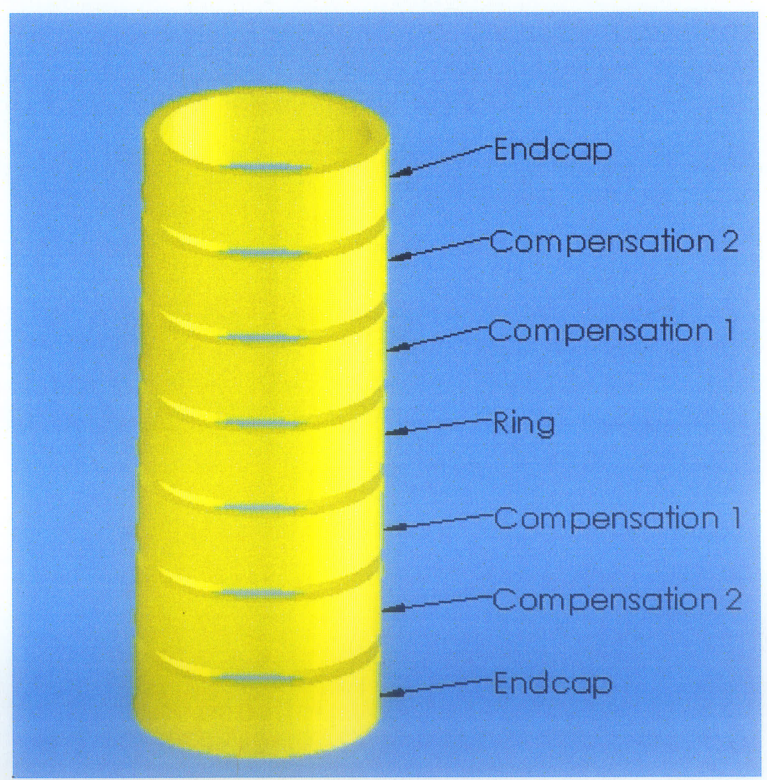

Figure 2.10: Cylindrical Penning trap. 
where

$$
d^{2}=\frac{1}{2} \cdot\left(z_{0}^{2}+\frac{r_{0}^{2}}{2}\right)
$$

From Eq. 2.26, the electric field in the trap is

$$
\vec{E}(x, y, z)=-\vec{\nabla} U(x, y, z)=\frac{U_{0}}{2 d^{2}}(x \hat{x}+y \hat{y}-2 z \hat{z})
$$

The magnetic field

$$
\vec{B}=B \hat{z}
$$

and the ion position is

$$
\vec{r}=x \hat{x}+y \hat{y}+z \hat{z}
$$

The ion motions in the magnetic field and electric field is given by

$$
m \ddot{\vec{r}}=q(\vec{E}+\dot{\vec{r}} \times \vec{B}) .
$$

By setting

$$
\omega_{z}=\sqrt{\frac{q U_{0}}{m d^{2}}} \quad \omega_{c}=\frac{q B}{m}
$$

Eq. 2.31 can be replaced by the following three equations

$$
\begin{gathered}
\ddot{x}-\frac{\omega_{z}^{2}}{2} x-\omega_{c} \dot{y}=0, \\
\ddot{y}-\frac{\omega_{z}^{2}}{2} y-\omega_{c}(-\dot{x})=0, \\
\ddot{z}-\frac{\omega_{z}^{2}}{2}(-2 z)=0 .
\end{gathered}
$$

The solution of Eq. 2.35 is

$$
z=A_{z} \cos \left(\omega_{z} t-\phi_{z}\right)
$$


It means the axial motion is a harmonic oscillation, with amplitude $A_{z}$ and phase $\phi_{z}$ determined by the initial axial position and velocity. By setting $u=x+y i$, the radial equations 2.33 and 2.34 become

$$
\ddot{u}-\omega_{c} \dot{u} i-\frac{\omega_{z}^{2}}{2} u=0 .
$$

Setting

$$
u=e^{-i \omega t}
$$

the characteristic frequencies $\omega_{ \pm}$can be obtained:

$$
\omega_{ \pm}=\frac{1}{2}\left(\omega_{c} \pm \sqrt{\omega_{c}^{2}-2 \omega_{z}^{2}}\right)
$$

The solution of Eq. 2.37 is

$$
u=A_{+} e^{-i \omega_{+} t}+A_{-} e^{-i \omega_{-} t}
$$

which yields the solutions for $x$ and $y$ :

$$
\begin{aligned}
& x=R_{-} \cos \left(\omega_{-} t-\phi_{-}\right)+R_{+} \cos \left(\omega_{+} t-\phi_{+}\right) \\
& y=-R_{-} \sin \left(\omega_{-} t-\phi_{-}\right)-R_{+} \sin \left(\omega_{+} t-\phi_{+}\right)
\end{aligned}
$$

Where $R_{-}$is called the radius of the magnetron motion, and $R_{+}$is called the radius of the reduced cyclotron motion.

\subsubsection{Quadrupole excitation}

The oscillating azimuthal quadrupole potential $[36,66]$ is created via a four-segmented ring electrode shown in Figure $2.11 ;+U$ is applied at one pair of the four-segmented 


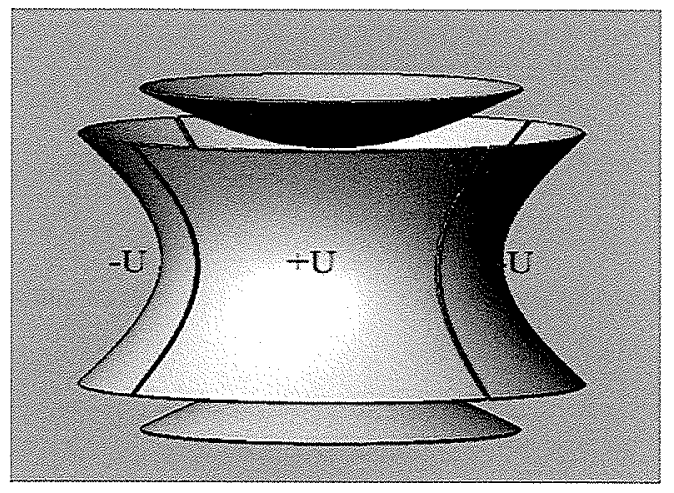

Figure 2.11: Hyperbolic Penning trap; the ring electrode is cut into four segments.

ring electrodes, and $-U$ is applied at another pair of the four-segmented ring electrodes. The RF signal $U$ is

$$
U=V_{r f} \cos \left(\omega_{q} t-\phi_{q}\right)
$$

$V_{r f}, \omega_{q}$ and $\phi_{q}$ are the amplitude, the frequency and the phase of the RF field. The quadrupole potential inside the trap is

$$
\phi_{q}=-\frac{V_{r f}}{2 a^{2}} \cos \left(\omega_{q} t-\phi_{q}\right)(x y)
$$

then the quadrupole field is given by

$$
\vec{E}=\frac{V_{r f}}{2 a^{2}} \cos \left(\omega_{q} t-\phi_{q}\right)(x \hat{x}+y \hat{y})
$$

By adding an RF azimuthal quadrupole field, similar to Eq. 2.33 and Eq. 2.34, the ion motion equations become

$$
\ddot{x}-\frac{\omega_{z}^{2}}{2}\left[x+\frac{V_{r f}}{2 a^{2}} \cos \left(\omega_{q} t-\phi_{q}\right) y\right]-\omega_{c} \dot{y}=0
$$


and

$$
\ddot{y}-\frac{\omega_{z}^{2}}{2}\left[y+\frac{V_{r f}}{2 a^{2}} \cos \left(\omega_{q} t-\phi_{q}\right) x\right]-\omega_{c}(-\dot{x})=0
$$

Solving the equations 2.46 and 2.47 , we obtain [36]

$$
x=R_{-}(t) \cos \left(\omega_{-} t-\phi_{-}\right)+R_{+}(t) \cos \left(\omega_{+} t-\phi_{+}\right)
$$

and

$$
y=-R_{-}(t) \sin \left(\omega_{-} t-\phi_{-}\right)-R_{+}(t) \sin \left(\omega_{+} t-\phi_{+}\right)
$$

where

$$
\begin{gathered}
R_{ \pm}(t)=\left\{R_{ \pm}(0) \cos \left(\omega_{B} t\right) \mp \frac{1}{2} \frac{R_{ \pm}(0)\left[i\left(\omega_{r f}-\omega_{c}\right)\right]+R_{\mp}(0) k_{0}^{ \pm}}{\omega_{B}} \times \sin \left(\omega_{B} t\right)\right\} e^{i \frac{1}{2}\left(\omega_{r f}-\omega_{c}\right) t} \\
\omega_{B}=\frac{1}{2} \sqrt{\left(\omega_{r f}-\omega_{c}\right)^{2}+k_{0}^{2}}, \\
k_{0}^{ \pm}=k_{0} e^{ \pm i \triangle \phi}, \\
k_{0}=\frac{V_{r f}}{2 a^{2}} \frac{q}{m} \frac{1}{\omega_{+}-\omega_{-}}, \\
\triangle \phi \\
\triangle \varphi-\left(\varphi_{+}+\varphi_{-}\right),
\end{gathered}
$$

$\varphi_{r f}, \varphi_{+}$and $\varphi_{-}$are the phases of the r.f. field and the two radial motions. The radial energy can be given by

$$
E_{r}=\frac{1}{2} m\left[\left(R_{+} \omega_{+}\right)^{2}+\left(R_{-} \omega_{-}\right)^{2}\right]
$$

and with $\omega_{+} \gg \omega_{-}$, Eq. 2.55 can be replaced by

$$
E_{r} \approx \frac{1}{2} m\left[\left(R_{+} \omega_{+}\right)^{2}\right]
$$


From 2.50 and 2.56, for the special case $R_{+}(0)=0$, the radial energy can be given by

$$
E_{r} \approx \frac{1}{4} m R_{-}^{2}(0) k_{0}^{2} \frac{\sin ^{2}\left(\omega_{B} t\right)}{\omega_{B}^{2}} .
$$

\subsubsection{Time of flight}

Figure 2.12 shows axial $B_{z}$ and radial $B_{r}$ field components of the $4 \mathrm{~T}$ magnet for the precision Penning trap at an off-axis distance of $0.5 \mathrm{~cm}$. The origin is located at the magnet center. After quadrupole excitation, when letting ions out of the Penning trap, the ions are accelerated by an axial force arising from the magnetic field gradient.

From Appendix A, the force $F$ depends on the radial energy $E_{r}$ and magnetic field gradient $\frac{\partial B}{\partial z}$, and is given by

$$
F=-\frac{E_{r}}{B} \frac{\partial B}{\partial z} \hat{z} .
$$

From Eq. 2.58 and Eq. 2.57, if changing the RF signal frequency $\omega_{r f}$ at the ring electrodes of the Penning trap, the radial energy $E_{r}$ will be changed. If $E_{r}$ reaches a maximum, then the axial force reaches a maximum too. With Eq. 2.58 and Eq. 2.57, by solving Eq. 2.59

$$
\frac{\partial F}{\partial \omega_{r f}}=0
$$

$\omega_{r f}=\omega_{c}$ can be obtained. This means that $E_{r}$ reaches a maximum only when $\omega_{r f}$ is equal to $\omega_{c}$. From Eq. 2.32, we can know the ion mass if we make the radial energy a maximum after quadrupole excitation. How do we know when $\omega_{r f}=\omega_{c}$ ? We can 
measure the time of flight,

$$
\operatorname{TOF}\left(\omega_{r f}\right)=\int_{z=0}^{z_{\text {detector }}} \sqrt{\frac{m}{2\left(E_{0}-q \cdot V(z)-\frac{E_{r}}{B} \cdot B(z)\right)}} d z,
$$

where $E_{0}$ is the ion energy after quadrupole excitation, $z_{\text {detector }}$ is the detector distance from the Penning trap center, $V(z)$ is the axial potential, $B$ is the magnetic field at the center of Penning trap, and $B(z)$ is the axial magnetic field, which changes with the position. When the time of flight is a minimum, we know the axial force on the ion is a maximum, and the RF signal frequency $\omega_{r f}$ must be equal to $\omega_{c}$. Also, from Eq. 2.57, $E_{r}$ is maximized when $\sin ^{2}\left(\omega_{B} T_{r f}\right)=1$. On resonance $\omega_{B}=\frac{k_{0}}{2}$ and therefore $T_{r f}=\frac{\pi}{k_{0}}$. Hence, via $k_{0}$, the optimum duration of the RF signal depends on the RF amplitude.

From the above, by means of a Penning trap and measuring the ion flight time, ion mass measurement is possible.

\subsection{Simulation for time of flight}

SIMION [67] is a very useful software; using it, we can simulate the same procedure that is used in a real measurement. The ion moves in an electric field and magnetic field, and SIMION calculates the ion trajectory by a fourth-order Runge-Kutta method (see Chapter 4). The result strongly depends on the time step. Only if we choose sufficiently small time steps, will the result correctly describe the experiment. The system is set up as shown in Figure 2.13; the dimensions are shown in Appendix B. DC voltages at the four ring electrodes of the Penning trap are the same and are 


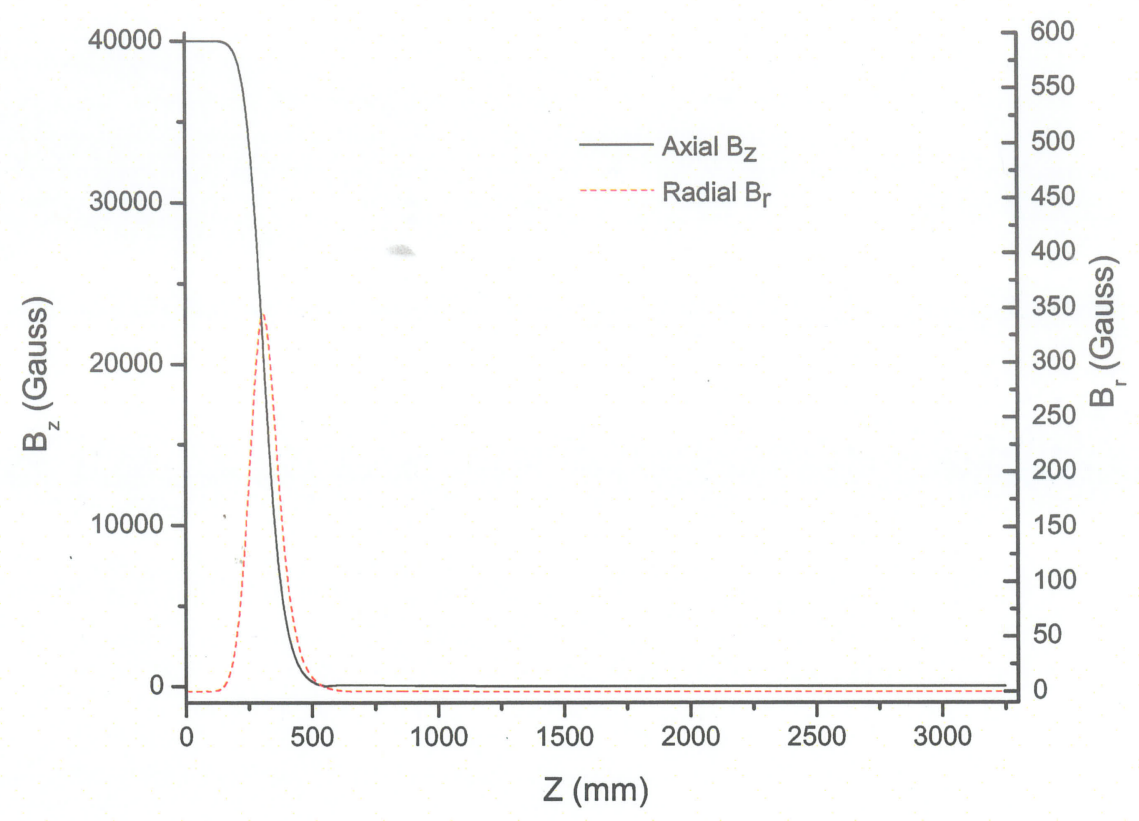

Figure 2.12: Axial magnetic field $B_{z}$ at an off-axis distance of $0 \mathrm{~mm}$ and radial magnetic field $B_{r}$ for the precision Penning trap at an off-axis distance of $5 \mathrm{~mm}$. The origin is located at the magnet center.

set to $-0.78 \mathrm{~V}$; the two endcap electrodes of the Penning trap are set to $1 \mathrm{~V}$; the electrodes next to the Penning trap are respectively set to $-40 \mathrm{~V},-2 \mathrm{~V},-1000 \mathrm{~V},-100 \mathrm{~V}$, $-1000 \mathrm{~V},-2150 \mathrm{~V}$. Initially, ${ }^{68} \mathrm{Ge}^{+}$is stopped at the trap center, then a dipolar signal is applied at the ring electrodes for $700 \mu \mathrm{s}$, so that ion can move out of the center of the trap. Next, a quadrupolar signal is applied at the ring electrodes for $400 \mathrm{~ms}$; the amplitudes of dipolar and quadrupolar are set to $0.8 \mathrm{~V}$ and $0.025 \mathrm{~V}$, the time of flight is recorded at axial position $738 \mathrm{~mm}$ when the ion passes this position finally, and the same process is repeated. The frequency of the quadrupolar signal is changed by 


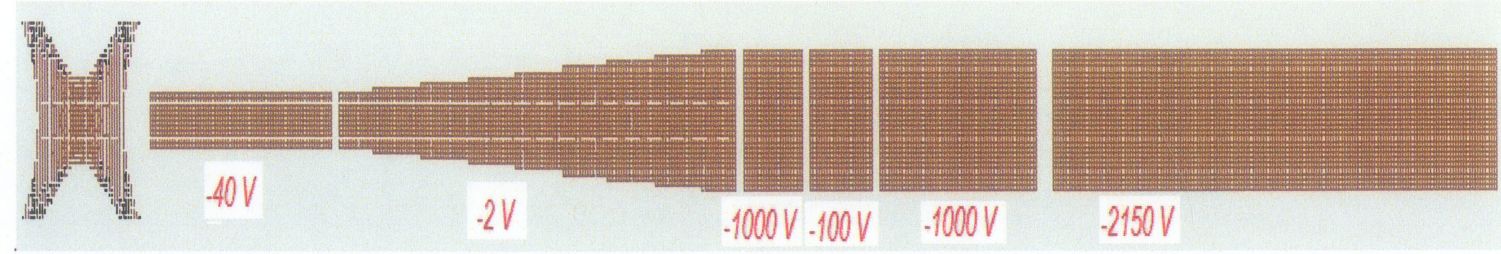

Figure 2.13: The system for time of flight.

a .PRG program shown in Appendix C. Figure 2.14 shows that the frequency where the time of flight is a minimum, is roughly 5681616.287 which is equal to $\omega_{c}$ in a 4 Tesla magnetic field. SIMION is a good tool for studying the Penning trap, but the correct time step is important; short time steps make the simulation time very long, whereas large time steps make the results inaccurate.

\subsection{Current status of TITAN}

In August 2007, TITAN got first beam from ISAC. Currently, the RFQ, the precision Penning trap for mass measurement, and the ion transport lines between them are fully operational and permit the measurement of singly charged ions. First results include the mass determination in a series of light radioactive nuclei such as ${ }^{8} \mathrm{He}$,

${ }^{11} \mathrm{Li}$ and ${ }^{11} \mathrm{Be}$, also referred to as 'halo nuclei' which have received a lot of attention recently. The EBIT has been installed on the TITAN platform, but is currently under repair. The cooler trap described in this thesis is entering the construction phase in summer 2008 . 


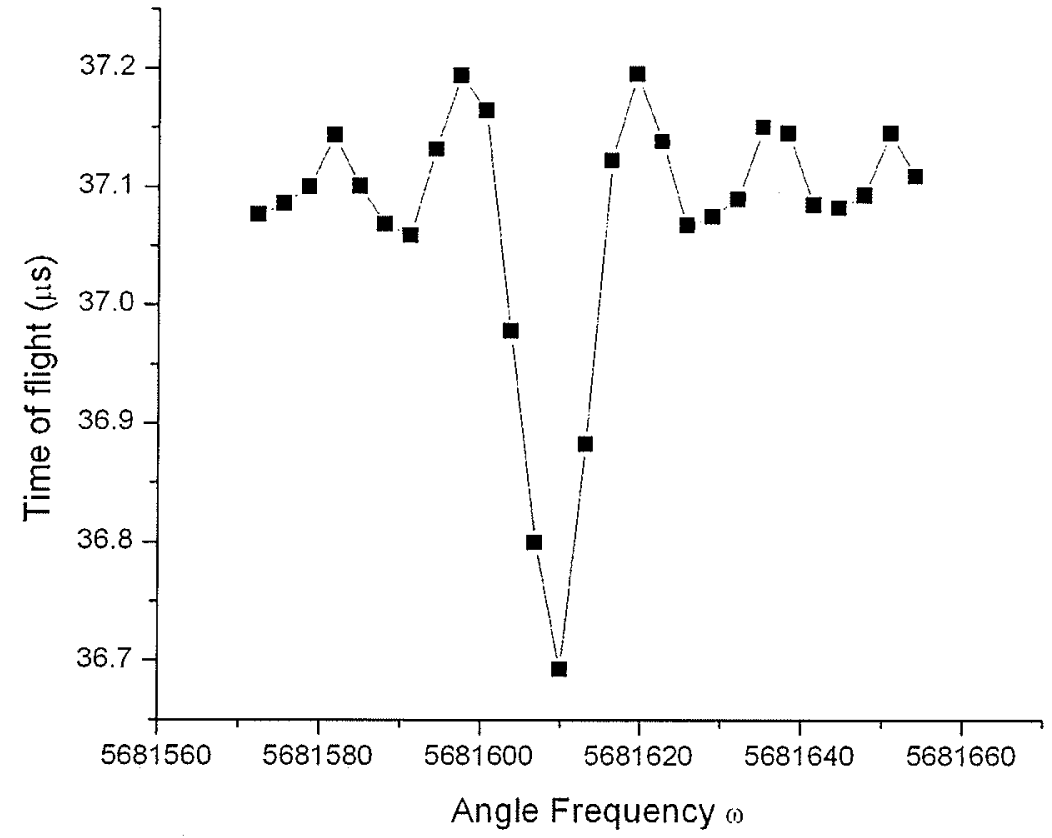

Figure 2.14: Time of flight from SIMION. 


\section{Chapter 3}

\section{COOLING TECHNIQUES AND ELECTRON-ION RECOMBINATION}

There are numerous beneficial effects $[68,69]$ brought about from cooling of ions.

First, losses of ions can be reduced and ions can be trapped in an ion trap for a long time; second, ions can be localized near the center of the trap, both of which increase the precision of measurements of ion masses; and finally, the Doppler shifts can be reduced in optical and $\gamma$-ray spectroscopy where the transition is broadened by Doppler shifts related to the ion velocity. Highly charged ions should be cooled down before they enter the Penning trap. There are a few methods to cool ions, but the charge state of highly charged ions must not be changed during the cooling process. Some of the following methods can be used to cool highly charged ions from an EBIT; some of them are not suitable for our purposes. 


\subsection{Cooling methods}

\subsubsection{Resistive cooling}

For this technique, an external tuned circuit is connected to the trap electrodes. By inducing electric currents in the external circuit, the moving ions dissipate energy. The induced current is given by $i=B_{1} q v_{z} / 2 z_{0}$, where $q$ is the ion charge, $v_{z}$ the ion velocity, and $z_{0}$ the half separation between the electrodes. For hyperboloidal Penning trap electrodes, $B_{1}=0.8[70,71]$. For parallel plate electrodes, $B_{1}=1$. The average rate of ion energy change $[72,68]$ can be written as

$$
\frac{d W_{z}}{d t}=-\left\langle i^{2} R_{\mathrm{eff}}\right\rangle=-\frac{B_{1}^{2} q^{2} R_{\mathrm{eff}} W_{z}}{4 m z_{0}^{2}}
$$

where $W_{z}$ is the total energy of the ion, $m$ is the ion mass, and $R_{\text {eff }}$ is the on-resonance impedance for the $L C$ circuit, given by

$$
R_{\mathrm{eff}}=Q /\left(\omega_{z} C\right)
$$

where $Q$ is the quality factor of the tuned circuit, and $\omega_{z}$ is the ion oscillation frequency. From Eqns. 3.1 and 3.2, the average rate of ion energy change is given by

$$
\frac{d W_{z}}{d t}=W_{z}(0) e^{-\frac{t}{\tau}}
$$

where

$$
\tau=\frac{4 m z_{0}^{2}}{B_{1}^{2} q^{2} R_{\mathrm{eff}}}=\frac{4 m z_{0}^{2} \omega_{z} C}{B_{1}^{2} q^{2} Q} .
$$


Eqns. 3.3 and 3.4 show that resistive cooling is most effective for ions with high charge-to-mass ratios, high $Q$ and small $Z_{0}$. A sufficiently high tuned circuit quality factor can only be achieved in a cryogenic environment $(4.2 \mathrm{~K})$ and results in narrow resonances. Hence this method is problematic for a broad-band cooler device (i.e. that cools HCIs with a large range of $q / m$ ) in a room-temperature vacuum environment.

\subsubsection{Evaporative cooling}

In an electron ion beam trap (EBIT), the ions are heated by the intense electron beam, and the ion energy rapidly increases. If the ions are not cooled in some way, they will boil out of the trap before they reach the desired high charge state. Evaporative cooling can be used to prevent this loss. If we inject low- $Z$ ions along with higher$Z$ ones, they are all trapped in the EBIT, collide with the hot electrons, and reach essentially the same energy. If the axial kinetic energy of an ion is more than the axial potential barrier $q V_{\text {well }}$, the ion will evaporate from the EBIT. The low- $Z$ ions tend to be fully stripped, but since they are low $Z$, the axial potential barrier, which is proportional to the charge, is lower for them, so the low- $Z$ ions escape from the EBIT before the heavier ions reach a high charge state. The equilibrium trapped-ion temperature [73] is given by

$$
T_{i} \approx 0.1 q V_{w e l l}
$$

where $T_{i}$ is the ion temperature in energy units. Each ion which escapes through the axial potential barrier removes an energy $q V_{\text {well }}$, which is much larger than the 
average thermal energy shown in Eq. 3.5. From the general relation of energy and

temperature, $W=\frac{3}{2} N_{i} k T_{i}$, we see that the new temperature is lower than before. The thermal energy removed by evaporation of the light, weakly trapped ions permits the heavy, highly charged ions to remain trapped. Evaporative cooling in the TITAN EBIT still needs to be investigated thoroughly and has the potential to make a significant contribution to the cooling process.

\subsubsection{Buffer gas cooling}

Ions interact with the buffer gas, transfer energy to the buffer gas by collision, and finally reach thermal equilibrium with the buffer gas. In practice, the interaction of ion and gas is very complicated. The interactions may be described by long-range, short-range and intermediate-range potentials. The long-range potential consists of a polarization potential, dispersion potential and electrostatic potential. The polarization potential arises from the interaction between the ions and the multipole moments induced in the gas by the ions. The dominant long-range potential is usually the ion-induced dipole potential which is proportional to $r^{-4}$. Electron fluctuations give rise to transient multipole moments which induce in-phase transient moments in another molecule or ion; the dispersion potential rises from the electrostatic interactions between these two sets of moments. The electrostatic potential arises from the interactions between the charge of the ion and the permanent multipole moments of the gas or the interactions between the permanent multipole moments of the ion and 
the permanent multipole moments of the gas. The short-range potential comes from the electronic charge clouds of an ion and an atom or molecule overlapping at short range. The intermediate-range potential includes both of the above, the potential arising from the effects of both Coulombic interaction and the overlapping electron clouds. The interaction potential form [74] is given by

$$
V(r)=\frac{n \varepsilon_{0}}{n(3+\gamma)-12(1+\gamma)}\left[\frac{12}{n}(1+\gamma)\left(\frac{r_{m}}{r}\right)^{n}-4 \gamma\left(\frac{r_{m}}{r}\right)^{6}-3(1-\gamma)\left(\frac{r_{m}}{r}\right)^{4}\right]
$$

where $n, \gamma, \varepsilon_{0}$, and $r_{m}$ are the parameters. This potential is called the $(n, 6,4)$ ionneutral potential [75]. The parameters depend on the kind of gas and ion. The $r^{-n}$ term represents the short-range repulsion of the interaction between an ion and an atom or molecule. The $r^{-6}$ term and $r^{-4}$ represent the attractive long-range interaction.

Buffer gas cooling is used in the radio frequency quadrupole $[76,77,78,79]$.

$$
\overrightarrow{V_{d}}=K \vec{E}
$$

where $V_{d}$ is the drift velocity of an ion in a gas, and $K$ is the mobility. $K$ is given by

$$
K=\frac{q}{N}\left(\frac{1}{3 \mu k T_{\text {eff }}}\right)^{1 / 2} \frac{1}{Q_{D}\left(T_{\text {eff }}\right)}
$$

where $\mu=m M /(m+M)$, and

$$
\frac{3}{2} k T_{\mathrm{eff}}=\frac{3}{2} k T+\frac{1}{2} M V_{d}^{2}
$$


where $M$ is the mass of the gas, $m$ is the mass of the ion, and $T$ is the temperature of gas. The one-temperature theory [74] gives

$$
Q_{D}\left(T_{\mathrm{eff}}\right)=\frac{1}{2}\left(k T_{\mathrm{eff}}\right)^{-3} \int_{0}^{\infty} \int_{0}^{\pi} 2 \pi(1-\cos \theta) b e^{\left(-\varepsilon / k T_{\mathrm{eff}}\right)} \varepsilon^{2} d b d \varepsilon
$$

where $b$ is the impact parameter, and

$$
\varepsilon=\frac{1}{2} \mu v_{r}^{2}
$$

where $v_{r}$ is the relative velocity, $\theta$ is given by

$$
\theta=\pi-2 b \int_{r_{0}}^{\infty}\left[1-\frac{b^{2}}{r^{2}}-\frac{V(r)}{\varepsilon}\right]^{-1 / 2} \frac{d r}{r^{2}}
$$

where $V(r)$ is the interaction potential given by Eq. 3.6, and $r_{0}$ is the distance of closest approach; it is given by

$$
1-\frac{b^{2}}{r_{0}^{2}}-\frac{V\left(r_{0}\right)}{\varepsilon}=0
$$

From the above equations, given a specific condition, the mobility can be calculated. Normally, the parameters can be obtained from experiment.

Viscous damping models can provide a simple description of neutral ion interactions. When the ion velocity is low, the damping force between the ion and buffer gas is simply given by Stokes' law, the damping force being proportional to the ion velocity. If the ion velocity is high, the relationship is more complicated. The damping force for low ion velocity is given by

$$
\vec{F}_{d}=-\delta \cdot m \cdot \vec{v}
$$


where $m$ and $v$ are the mass and the velocity of the ion, and the damping coefficient is given by

$$
\delta=\frac{q}{m} \cdot \frac{1}{K_{0}} \cdot \frac{p / p_{0}}{T / T_{0}}
$$

where $q$ is the charge of ion, and $p$ and $T$ are the pressure and temperature of gas respectively; $p_{0}$ and $T_{0}$ are respectively the pressure and temperature of the gas for the standard reference conditions, $p_{0}=1$ bar, and $T_{0}=297 \mathrm{~K} . K_{0}$ is the reduced ion mobility, which is the mobility of the ions for standard temperature $T_{0}$ and pressure $p_{0}$.

When cooling highly charged ions using a buffer gas, the charge state of the highly charged ions will be changed, so buffer gas cooling can usually not be used for this purpose, but is widely and successfully used with singly charged ions.

\subsubsection{Laser cooling}

For laser cooling $[80,81]$, the laser is tuned to a frequency slightly below an electronic transition in the atom. Because of the Doppler effect, for atoms moving in the direction opposite to the laser beam, the frequency of the laser is shifted into resonance with the transition, and the atoms absorb photons and lose momentum equal to the momentum of the photons. Furthermore, because the excited atoms emit photons in a random direction, there is no net momentum transfer from the de-excitation when averaged over many excitation cycles. The net result is that the atoms lose energy by absorbing photons but don't increase energy by reemitting the photons, so the 
hot atoms cool down. The lowest achievable temperature of the atom is given by the Doppler cooling limit [82, 83],

$$
T_{D}=\hbar \gamma / 2 k_{B}
$$

where $\gamma^{-1}$ is the radiative lifetime of the laser cooling transition, $\hbar$ is constant, and $k_{B}$ is Boltzmann's constant.

Laser cooling is very effective; the cooling temperature which can be reached is much less than $1 \mathrm{~K}$. Direct laser cooling of an ion species requires a suitable electronic level structure and the required laser wavelengths are highly element-specific, ruling this method out as a general-purpose HCI cooling technique. However, sympathetic cooling of arbitrary species with laser-cooled $\mathrm{Li}, \mathrm{Be}, \mathrm{Mg}$, or $\mathrm{Ca}$ ions can be envisioned. It would apply to cooling HCI to energies significantly below the goal of the present setup, and would still rely on pre-cooling via electrons as discussed below.

\subsubsection{Sympathetic cooling}

Sympathetic cooling is a method whereby cold electrons, protons or other ions undergo energy-exchanging collisions with hot ions.

\section{Proton cooling}

Because of binary collisions between cold protons and ions, the ions lose energy. The rate of energy loss has been studied by many groups $[84,85,86,87,88]$. When one proton collides with an ion with collision cross section $\sigma$, the energy exchange of the 
elastic Coulomb collision is $\delta E(V, W)$, where $W$ is the proton velocity, and $V$ is the ion velocity. Considering the proton velocity distribution $f(W)$ (normally a Maxwellian distribution) corresponding to a proton temperature $T_{p}$, the energy exchange is given by

$$
d E=\int \delta E \sigma(|V-W|) f(W) d W d t
$$

Using the relation between energy and temperature, $E=\frac{3}{2} k T$, the time evolution of the ion and proton energies in a simple model $[84,87,88]$ are given by

$$
\begin{aligned}
\frac{d}{d t} T_{i} & =-\frac{1}{\tau_{i}}\left(T_{i}-T_{p}\right) \\
\frac{d}{d t} T_{p} & =\frac{1}{\tau_{i}} \frac{N_{i}}{N_{p}}\left(T_{i}-T_{p}\right) \\
\tau_{i} & =\frac{3\left(4 \pi \varepsilon_{0}\right)^{2} m_{p} m_{i} c^{3}}{8 \sqrt{2 \pi} n_{p} Z^{2} e^{4} \ln (\Lambda)}\left(\frac{k_{B} T_{i}}{m_{i} c^{2}}+\frac{k_{B} T_{p}}{m_{p} c^{2}}\right)^{\frac{3}{2}} \\
\ln (\Lambda) & =\ln \left(4 \pi\left(\frac{\varepsilon_{0} k_{B}}{e^{2}}\right)^{\frac{3}{2}} \frac{1}{Z} \sqrt{\frac{T_{p}}{n_{p}}}\left(T_{p}+\frac{m_{p}}{m_{i}} T_{i}+2 \sqrt{\frac{m_{p}}{m_{i}}} \sqrt{T_{p} T_{i}}\right)\right)
\end{aligned}
$$

where $T_{i}, m_{i}, Z$, and $N_{i}$ are respectively ion temperature, ion mass, ion charge, and the number of ions, and $T_{p}, n_{p}, m_{p}$, and $N_{p}$ are respectively proton temperature, proton density, proton mass, and number of protons. Note that this simple model ignores the influence of the magnetic field on the collision dynamics. With the magnetic field included, no analytic expression is available, instead extensive numerical simulations need to be employed [89]. The validity of our approach is discussed further at the end of this chapter.

With the initial conditions shown in Table 3.1, Figure 3.1 shows the energy evolution of various highly charged ions during the proton-HCI cooling process. The 
Table 3.1: Initial conditions for the energy evolution of various highly charged ions during the proton-HCI cooling process.

\begin{tabular}{|l|l|}
\hline proton density $\left(\mathrm{cm}^{-3}\right)$ & $10^{8}$ \\
\hline proton number & $10^{8}$ \\
\hline initial proton temperature $(\mathrm{eV})$ & 1 \\
\hline number of HCI & $10^{3}$ \\
\hline initial energy of $\mathrm{HCI}(\mathrm{eV} / q)$ & 500 \\
\hline
\end{tabular}
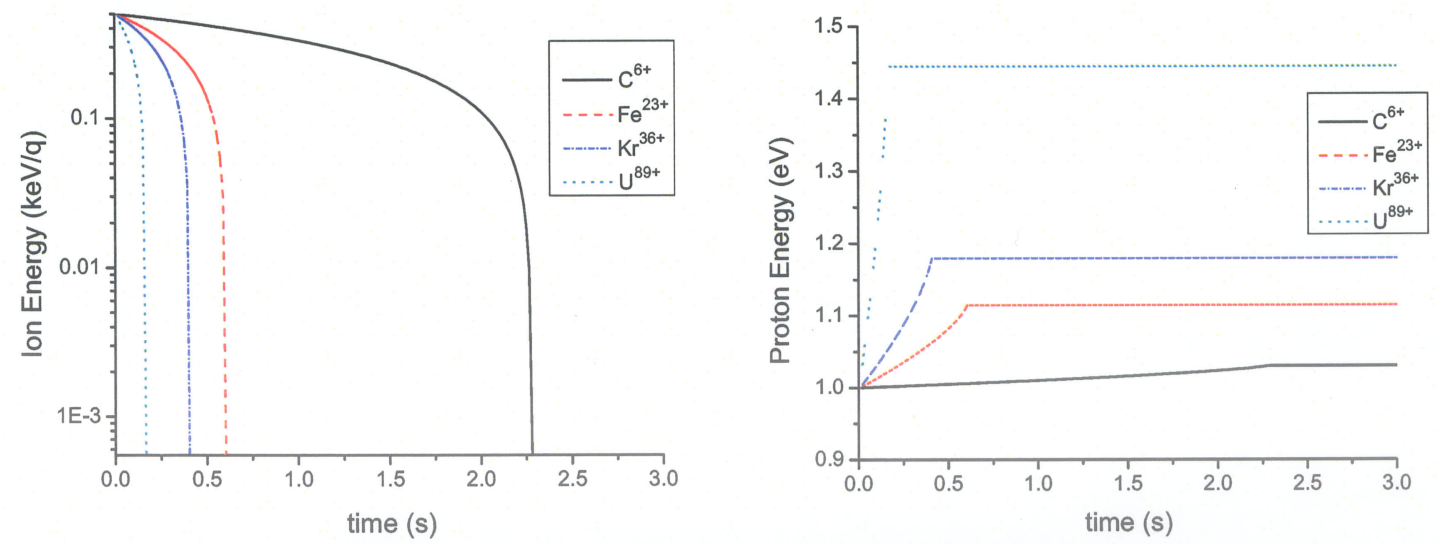

Figure 3.1: Energy evolution of vari- Figure 3.2: Energy evolution of protons ous highly charged ions (HCI) during the during the proton-HCI cooling process. proton-HCI cooling process. 
cooling time is about $0.15 \mathrm{~s}-2.3 \mathrm{~s}$, depending on the ion species. Figure 3.2 shows the energy evolution of protons during the proton-HCI cooling process. By energy conservation, if ions lose energy the protons gain energy.

Table 3.2: Initial conditions for the energy dependence of $\mathrm{Kr}^{36+}$ on the ratio of the number of HCI $\left(N_{i}\right)$ to the number of protons $\left(N_{p}\right)$.

\begin{tabular}{|l|l|}
\hline proton density $\left(\mathrm{cm}^{-3}\right)$ & $10^{8}$ \\
\hline proton number & $10^{8}$ \\
\hline initial proton temperature $(\mathrm{eV})$ & 1 \\
\hline initial energy of $\mathrm{Kr}^{36+}(\mathrm{eV} / \mathrm{q})$ & 500 \\
\hline
\end{tabular}
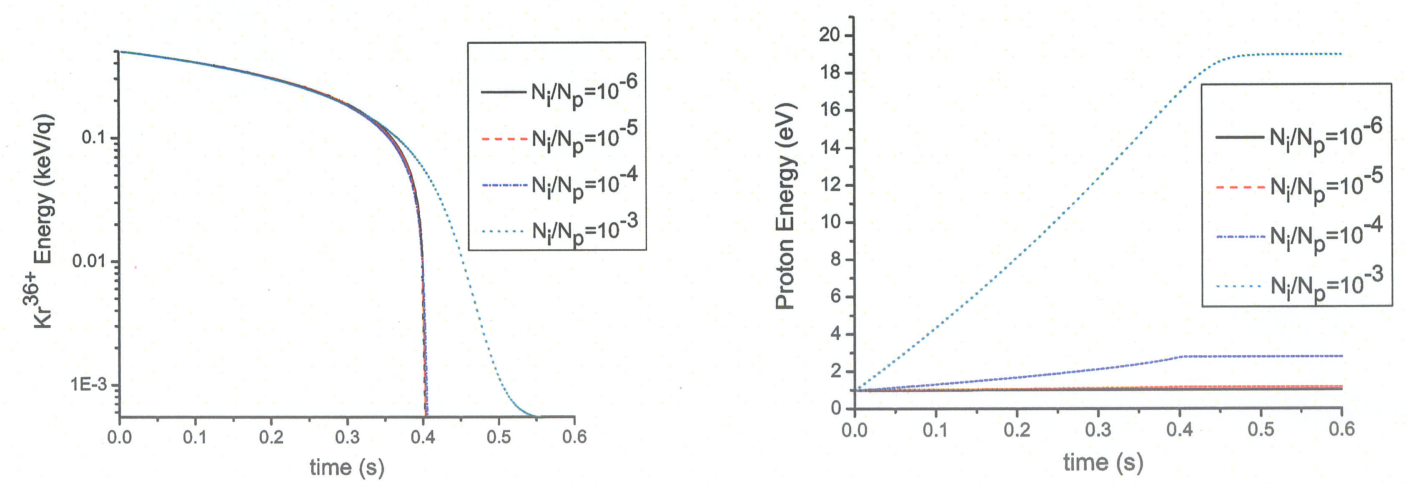

Figure 3.3: Energy dependence of $\mathrm{Kr}^{36+}$ on the ratio of the number of HCI $\left(N_{i}\right)$ to the number of protons $\left(N_{p}\right)$.

Figure 3.4: Energy dependence of protons on the ratio of the number of $\mathrm{HCI}$ $\left(N_{i}\right)$ to the number of protons $\left(N_{p}\right)$.

With the initial conditions shown in Table 3.2, Figure 3.3 shows the energy dependence of $\mathrm{Kr}^{36+}$ on the ratio of the number of HCI $\left(N_{i}\right)$ to the number of protons 
$\left(N_{p}\right)$. Figure 3.4 shows the energy dependence of protons on the ratio of the number of HCI $\left(N_{i}\right)$ to the number of protons $\left(N_{p}\right)$. The cooling time is about $0.4 \mathrm{~s}-0.5 \mathrm{~s}$, depending on the ion number.

Table 3.3: Initial conditions for the energy evolution of $\mathrm{Kr}^{36+}$ with different proton density and proton numbers during the proton $-\mathrm{Kr}^{36+}$ cooling process.

\begin{tabular}{|l|l|}
\hline number of $\mathrm{Kr}^{36+}$ & $10^{3}$ \\
\hline initial proton temperature $(\mathrm{eV})$ & 1 \\
\hline initial energy of $\mathrm{Kr}^{36+}(\mathrm{eV} / \mathrm{q})$ & 500 \\
\hline
\end{tabular}

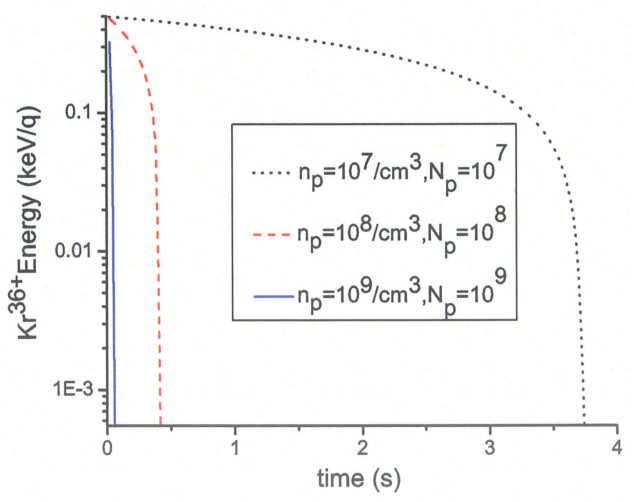

Figure 3.5: Energy evolution of $\mathrm{Kr}^{36+}$ with different proton density and proton numbers during the proton $-\mathrm{Kr}^{36+}$ cooling process.

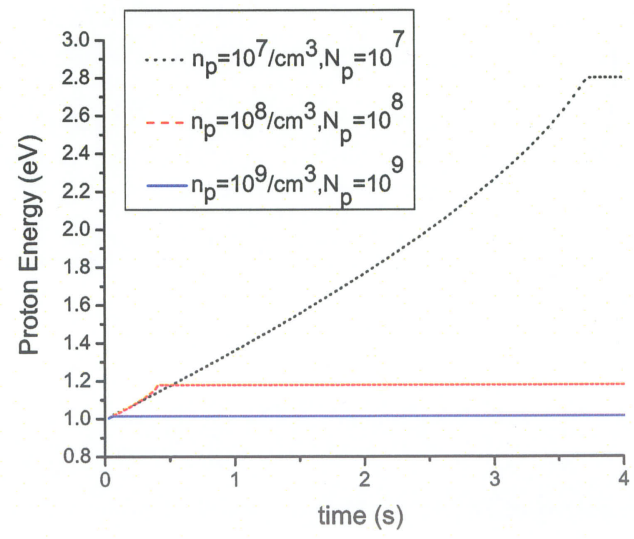

Figure 3.6: Energy evolution of protons with different proton density and proton numbers during the proton $-\mathrm{Kr}^{36+}$ cooling process.

With the initial conditions shown in Table 3.3, Figure 3.5 shows the energy evolution of $\mathrm{Kr}^{36+}$ with different proton density and proton numbers during the 
proton $-\mathrm{Kr}^{36+}$ cooling process. The cooling time is about $0.04 \mathrm{~s}-3.7 \mathrm{~s}$. Figure 3.6 shows the energy evolution of protons with different proton density and proton numbers during the proton $-\mathrm{Kr}^{36+}$ cooling process

Table 3.4: Initial conditions for the energy evolution of $\mathrm{Kr}^{36+}$ with different initial $\mathrm{Kr}^{36+}$ energy during the proton $-\mathrm{Kr}^{36+}$ cooling process.

\begin{tabular}{|l|l|}
\hline proton density $\left(\mathrm{cm}^{-3}\right)$ & $10^{8}$ \\
\hline proton number & $10^{8}$ \\
\hline initial proton temperature $(\mathrm{eV})$ & 1 \\
\hline number of $\mathrm{Kr}^{36+}$ & $10^{3}$ \\
\hline
\end{tabular}

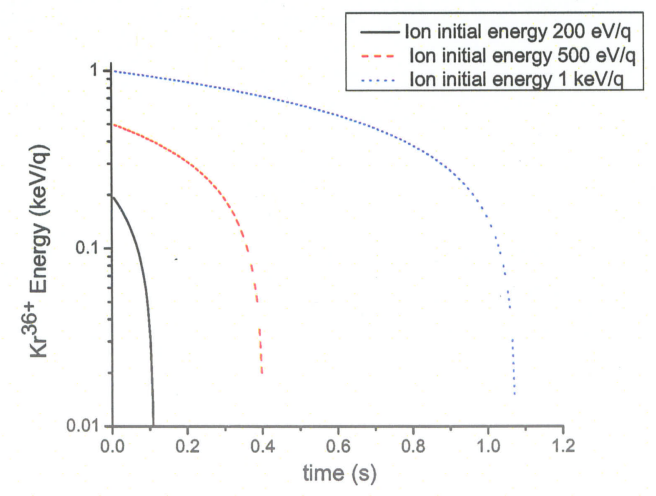

Figure 3.7: Energy evolution of $\mathrm{Kr}^{36+}$ with different initial $\mathrm{Kr}^{36+}$ energy during the proton $-\mathrm{Kr}^{36+}$ cooling process.

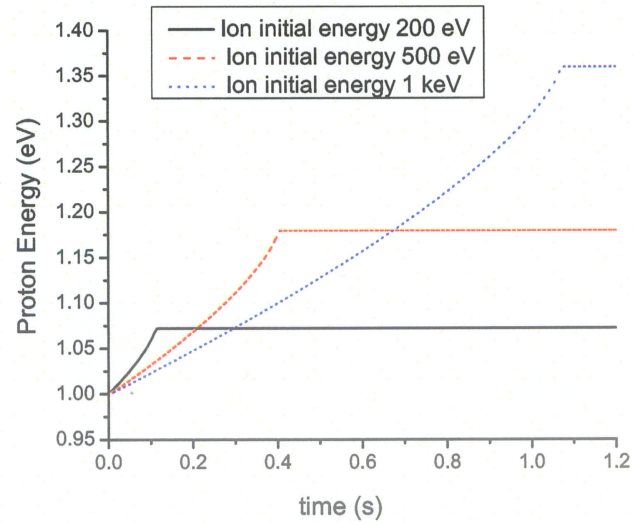

Figure 3.8: Energy evolution of protons with different initial $\mathrm{Kr}^{36+}$ energy during the proton $-\mathrm{Kr}^{36+}$ cooling process.

With the initial conditions shown in Table 3.4, Figure 3.7 shows the energy evo- 
lution of $\mathrm{Kr}^{36+}$ with different initial $\mathrm{Kr}^{36+}$ energy during the proton- $\mathrm{Kr}^{36+}$ cooling process. The cooling time is about $0.1 \mathrm{~s}-1.0 \mathrm{~s}$. Figure 3.8 shows the energy evolution of protons with different initial $\mathrm{Kr}^{36+}$ energy during the proton- $\mathrm{Kr}^{36+}$ cooling process.

Table 3.5: Initial conditions for the energy evolution of $\mathrm{Kr}^{36+}$ with different initial proton energy during the proton $-\mathrm{Kr}^{36+}$ cooling process.

\begin{tabular}{|l|l|}
\hline proton density $\left(\mathrm{cm}^{-3}\right)$ & $10^{8}$ \\
\hline proton number & $10^{8}$ \\
\hline initial energy of $\mathrm{Kr}^{36+}(\mathrm{eV} / q)$ & 500 \\
\hline number of $\mathrm{Kr}^{36+}$ & $10^{3}$ \\
\hline
\end{tabular}
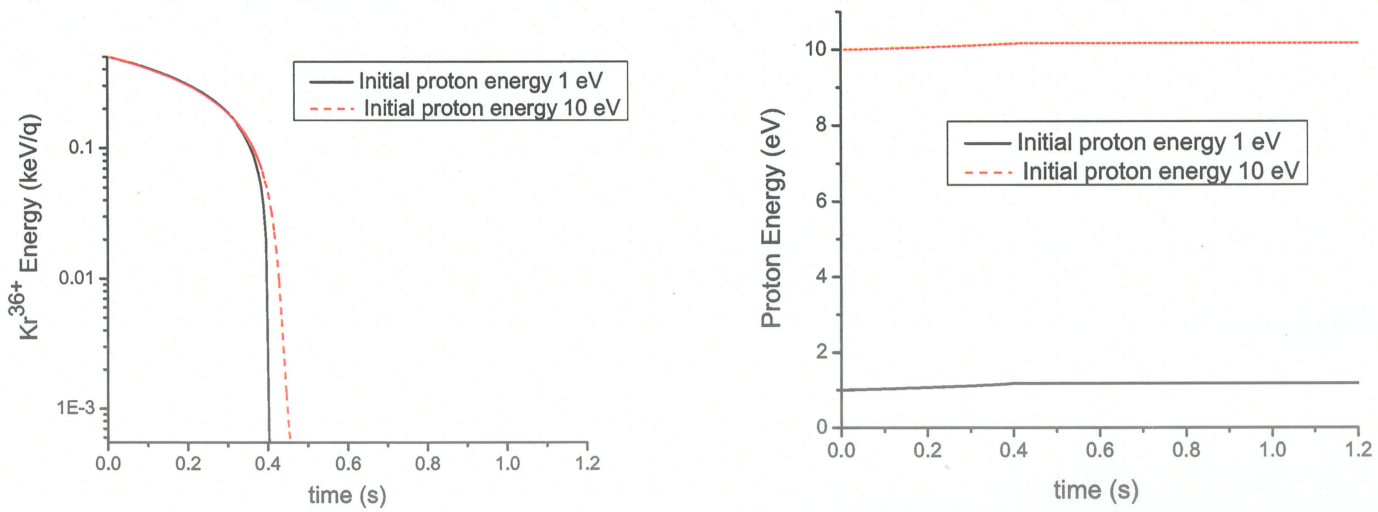

Figure 3.9: Energy evolution of $\mathrm{Kr}^{36+}$ Figure 3.10: Energy evolution of protons with different initial proton energy dur- with different initial proton energy during the proton $-\mathrm{Kr}^{36+}$ cooling process. ing the proton $-\mathrm{Kr}^{36+}$ cooling process.

With the initial conditions shown in Table 3.5, Figure 3.9 shows the energy evo- 
lution of $\mathrm{Kr}^{36+}$ with different initial proton energy during the proton- $\mathrm{Kr}^{36+}$ cooling process. The cooling time is about $0.4 \mathrm{~s}$, depending on the proton initial energy. Figure 3.10 shows the energy evolution of protons with different initial proton energy during the proton $-\mathrm{Kr}^{36+}$ cooling process.

Electron cooling

Similar to proton cooling, electron cooling uses electrons to cool the ions, but the electron is much lighter than the proton, and hence has a much larger charge-to-mass ratio $q / m$. In magnetic fields of a few Tesla, the emission of synchrotron radiation becomes significant and dampens the electron motion transverse to the magnetic field lines at time scales of seconds or faster (because the emitted power of synchrotron radiation scales inversely with the 4 th power of the particle mass, this effect is completely negligible for protons). Here, the dissipation via synchrotron radiation has to be taken into account. The time evolution of the ion and electron energy, which is identical to Eq. 3.18 with the exception of the added synchrotron radiation term, is given by $[90,91,92]$

$$
\begin{aligned}
\frac{d}{d t} T_{i} & =-\frac{1}{\tau_{i}}\left(T_{i}-T_{e}\right) \\
\frac{d}{d t} T_{e} & =\frac{1}{\tau_{i}} \frac{N_{i}}{N_{e}}\left(T_{i}-T_{e}\right)-\frac{1}{\tau_{e}}\left(T_{e}-T_{r e s}\right) \\
\tau_{i} & =\frac{3\left(4 \pi \varepsilon_{0}\right)^{2} m_{e} m_{i} c^{3}}{8 \sqrt{2 \pi} n_{e} Z^{2} e^{4} \ln (\Lambda)}\left(\frac{k_{B} T_{i}}{m_{i} c^{2}}+\frac{k_{B} T_{e}}{m_{e} c^{2}}\right)^{\frac{3}{2}} \\
\tau_{e} & =\frac{3 \pi \varepsilon_{0} m_{e} c^{3}}{e^{2} \omega_{c}^{2}}
\end{aligned}
$$




$$
\begin{aligned}
\omega_{c} & =\frac{e B}{m_{e}} \\
\ln (\Lambda) & =\ln \left(4 \pi\left(\frac{\varepsilon_{0} k_{B}}{e^{2}}\right)^{\frac{3}{2}} \frac{1}{Z} \sqrt{\frac{T_{e}}{n_{e}}}\left(T_{e}+\frac{m_{e}}{m_{i}} T_{i}+2 \sqrt{\frac{m_{e}}{m_{i}}} \sqrt{T_{e} T_{i}}\right)\right)
\end{aligned}
$$

where $T_{i}, m_{i}, Z$, and $N_{i}$ are respectively ion temperature, ion mass, ion charge, and the number of ions. $T_{e}, n_{e}, m_{e}, \omega_{c}, N_{e}$, and $\tau_{e}$ are respectively electron temperature, electron density, electron mass, electron cyclotron frequency, number of electrons, and electron synchrotron radiation time constant, $B$ is the magnetic field strength, and $T_{\text {res }}$ is the surrounding temperature (typically $293 \mathrm{~K}$ for a room-temperature vacuum vessel). Again, the influence of the magnetic field on the collision dynamics is neglected, but the field is taken into account in terms of the synchrotron radiation.

Table 3.6: Initial conditions for the energy evolution of various highly charged ions during the electron-HCI cooling process.

\begin{tabular}{|l|c|}
\hline electron density $\left(\mathrm{cm}^{-3}\right)$ & $10^{7}$ \\
\hline electron number & $10^{7}$ \\
\hline initial electron temperature (K) & 300 \\
\hline number of HCI & $10^{3}$ \\
\hline initial energy of HCI $(\mathrm{eV} / q)$ & 500 \\
\hline
\end{tabular}

With the initial conditions shown in Table 3.6, Figure 3.11 shows the energy evolution of $\mathrm{C}^{6+}, \mathrm{Fe}^{23+}, \mathrm{Kr}^{36+}$, and $\mathrm{U}^{89+}$ during the electron-HCI cooling process. 


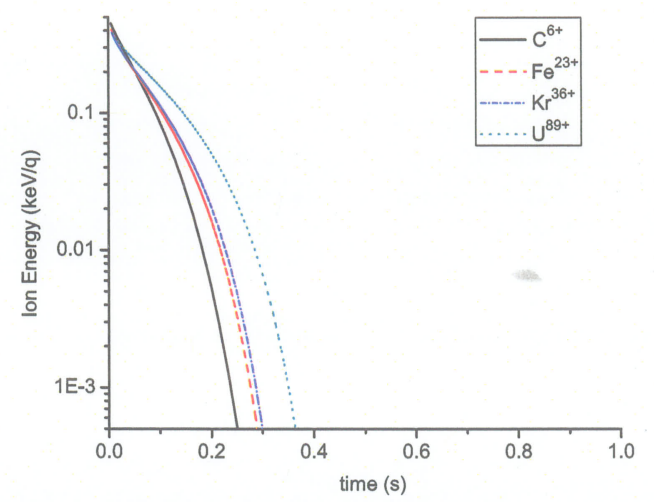

Figure 3.11: Energy evolution of vari- Figure 3.12: Energy evolution of elecous highly charged ions (HCI) during the trons during the electron-HCI cooling electron-HCI cooling process.

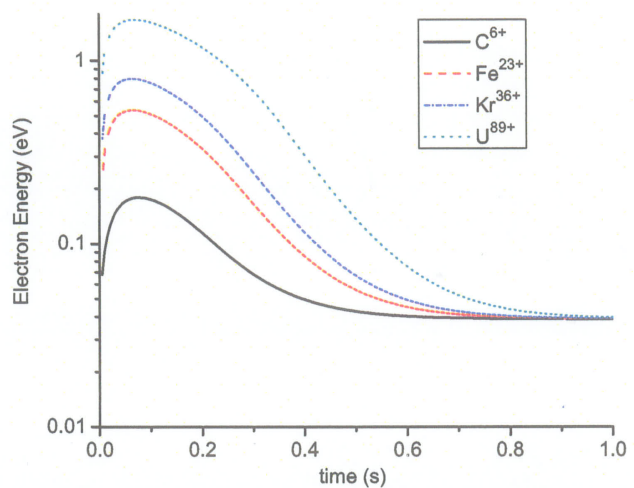
process.

The cooling time is about $0.24 \mathrm{~s}-0.35 \mathrm{~s}$, depending on the ion species. Figure 3.12 shows the energy evolution of electrons during the electron-HCI cooling process. Because of energy conservation, the electron energy increases when the ion energy decreases during the electron-HCI cooling process, but via synchrotron radiation process, the electron energy drops too during the electron-HCI cooling process.

Table 3.7: Initial conditions for the energy dependence of $\mathrm{Kr}^{36+}$ on the ratio of the number of HCI $\left(N_{i}\right)$ to the number of electrons $\left(N_{e}\right)$.

\begin{tabular}{|l|l|}
\hline electron density $\left(\mathrm{cm}^{-3}\right)$ & $10^{7}$ \\
\hline electron number & $10^{7}$ \\
\hline initial electron temperature $(\mathrm{K})$ & 300 \\
\hline initial energy of $\mathrm{Kr}^{36+}(\mathrm{eV} / \mathrm{q})$ & 500 \\
\hline
\end{tabular}



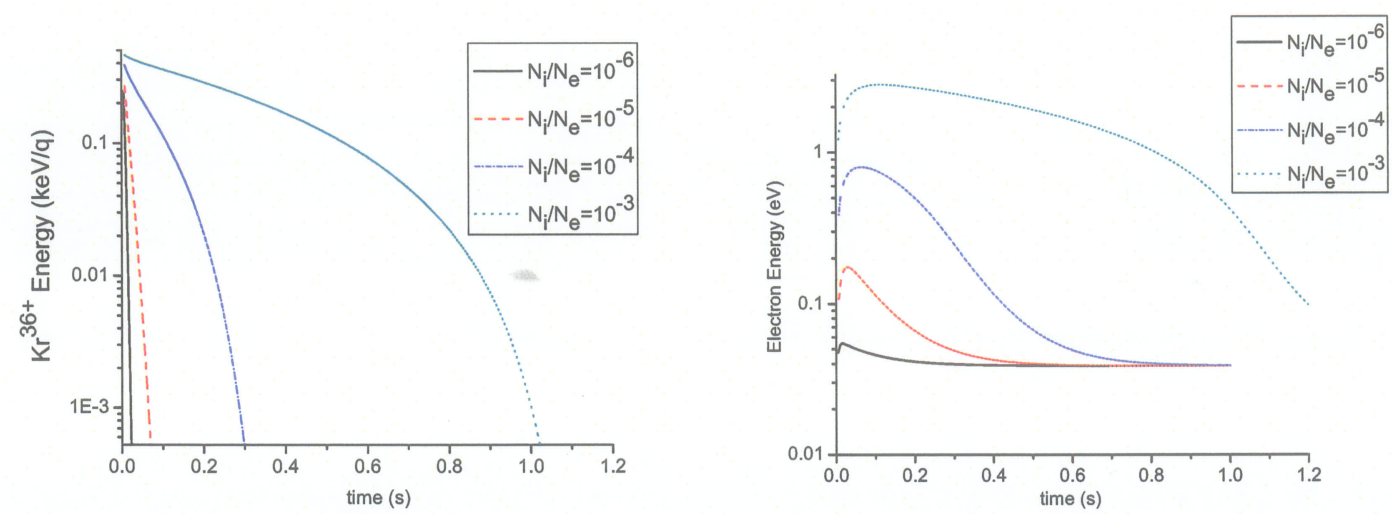

Figure 3.13: Energy dependence of $\mathrm{Kr}^{36+}$ on the ratio of the number of HCI $\left(N_{i}\right)$ to the number of electrons $\left(N_{e}\right)$.

Figure 3.14: Energy dependence of electrons on the ratio of the number of $\mathrm{HCI}$ $\left(N_{i}\right)$ to the number of electrons $\left(N_{e}\right)$.

With the initial conditions shown in Table 3.7, Figure 3.13 shows the energy dependence of $\mathrm{Kr}^{36+}$ on the ratio of the number of $\mathrm{HCI}\left(N_{i}\right)$ to the number of electrons $\left(N_{e}\right)$. The cooling time is about $0.02 \mathrm{~s}-1.0 \mathrm{~s}$, depending on the ion number. Figure 3.14 shows the energy dependence of electrons on the ratio of the number of HCI $\left(N_{i}\right)$ to the number of electrons $\left(N_{e}\right)$.

Table 3.8: Initial conditions for the energy evolution of $\mathrm{Kr}^{36+}$ with different initial $\mathrm{Kr}^{36+}$ energy during the electron $-\mathrm{Kr}^{36+}$ cooling process.

\begin{tabular}{|l|l|}
\hline electron density $\left(\mathrm{cm}^{-3}\right)$ & $10^{7}$ \\
\hline electron number & $10^{7}$ \\
\hline initial electron temperature $(\mathrm{K})$ & 300 \\
\hline number of $\mathrm{Kr}^{36+}$ & $10^{3}$ \\
\hline
\end{tabular}




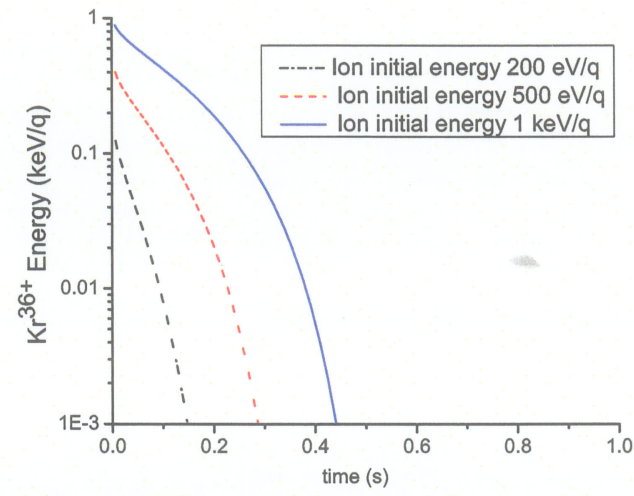

Figure 3.15: Energy evolution of $\mathrm{Kr}^{36+}$ with different initial $\mathrm{Kr}^{36+}$ energy during the electron $-\mathrm{Kr}^{36+}$ cooling process.

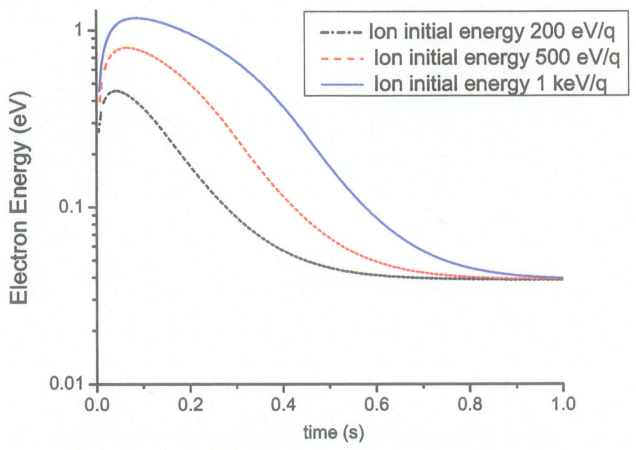

Figure 3.16: Energy evolution of electrons with different initial $\mathrm{Kr}^{36+}$ energy during the electron- $\mathrm{Kr}^{36+}$ cooling process.

With the initial conditions shown in Table 3.8, Figure 3.15 shows the energy evolution of $\mathrm{Kr}^{36+}$ with different initial $\mathrm{Kr}^{36+}$ energy during the electron- $\mathrm{Kr}^{36+}$ cooling process. The cooling time is about $0.15 \mathrm{~s}-0.44 \mathrm{~s}$, depending on the initial $\mathrm{Kr}^{36+}$ energy. Figure 3.16 shows the energy evolution of electrons with different initial $\mathrm{Kr}^{36+}$ energy during the electron- $\mathrm{Kr}^{36+}$ cooling process.

Table 3.9: Initial conditions for the energy evolution of $\mathrm{Kr}^{36+}$ with different surrounding temperature during the electron $-\mathrm{Kr}^{36+}$ cooling process.

\begin{tabular}{|l|l|}
\hline electron density $\left(\mathrm{cm}^{-3}\right)$ & $10^{7}$ \\
\hline electron number & $10^{7}$ \\
\hline number of $\mathrm{Kr}^{36+}$ & $10^{3}$ \\
\hline initial energy of $\mathrm{Kr}^{36+}(\mathrm{eV} / \mathrm{q})$ & 500 \\
\hline
\end{tabular}




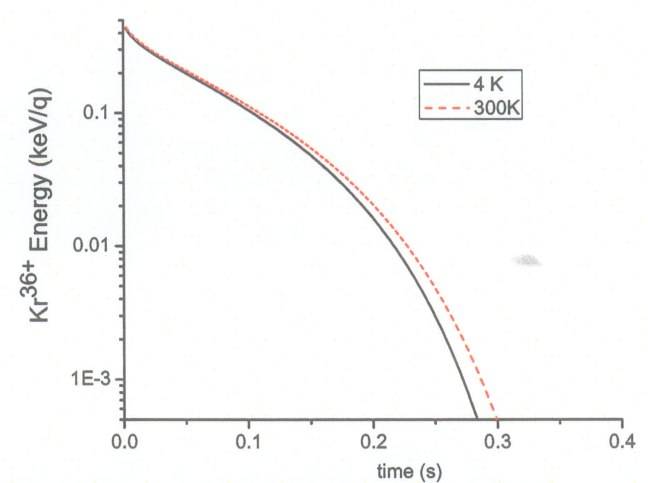

Figure 3.17: Energy evolution of $\mathrm{Kr}^{36+}$ with different surrounding temperature during the electron- $\mathrm{Kr}^{36+}$ cooling process.

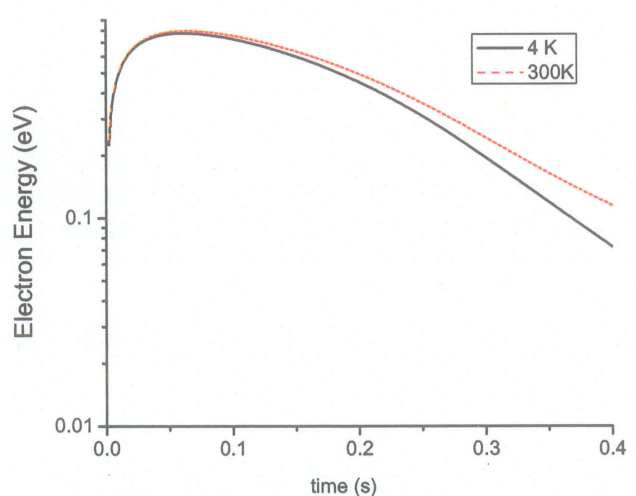

Figure 3.18: Energy evolution of electrons with different surrounding temperature during the electron- $\mathrm{Kr}^{36+}$ cooling process.

With the initial conditions shown in Table 3.9, Figure 3.17 shows the energy evolution of $\mathrm{Kr}^{36+}$ with different surrounding temperature during the electron- $\mathrm{Kr}^{36+}$ cooling process. The cooling time is about $0.3 \mathrm{~s}$. It seems that they are not much different at both surrounding temperature of $4 \mathrm{~K}$ or $300 \mathrm{~K}$. Figure 3.18 shows the energy evolution of electrons with different surrounding temperature during the electron- $\mathrm{Kr}^{36+}$ cooling process.

\subsection{Electron-ion recombination}

Electron-ion recombination processes in a cooler trap are very important to the cooling process, as such processes may alter the charge state. Electron-ion recombination has three modes [93, 94]: radiative recombination, dielectronic recombination [95, 96, 97] and three-body recombination [98]. 


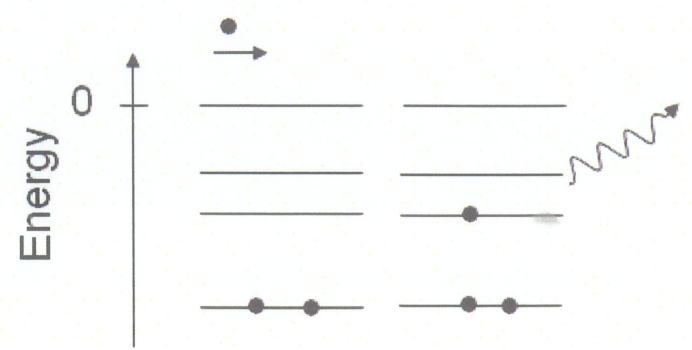

Radiative recombination

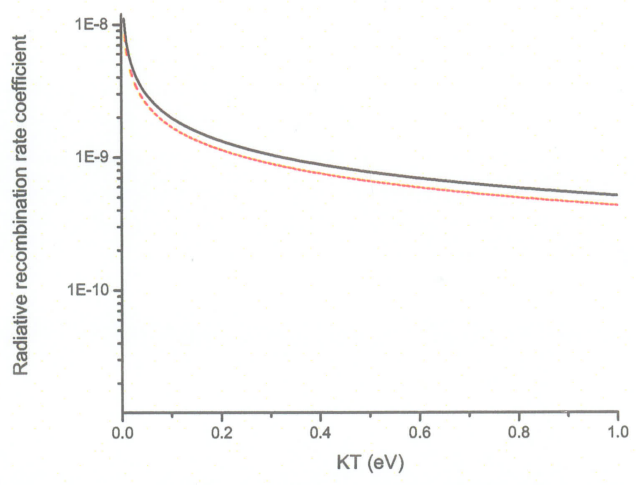

Figure 3.20: $\mathrm{Fe}^{23+}$ radiative recombination coefficient.

Figure 3.19 shows the radiative recombination $(\mathrm{RR})$ process; it is the inverse process of photoionization; it can occur when an ion interacts with an electron and the electron is captured directly into a vacant bound state with the emission of a photon that carries away the excess energy. The radiative recombination process can be written as

$$
A^{q+}+e^{-} \rightarrow A^{(q-1)+}+h \nu,
$$

where $h \nu$ is the emitted photon. The radiative recombination rate per ion, $R$, is conveniently described by the rate coefficient $\alpha_{R R}$, such that $R=\alpha_{R R} n_{e}$. The radiative recombination coefficient can be approximated as [94]

$$
\alpha_{R R}=\left[1.92 \times 10^{-13} \frac{\mathrm{cm}^{3}}{\mathrm{~s}}\right] Z^{2} \sqrt{\frac{e V}{T}}\left[\ln \left(\frac{5.66 Z}{\sqrt{T}}\right)+0.196\left(\frac{T}{Z^{2} e V}\right)^{1 / 3}\right]
$$

where $Z$ is the ion charge number and $T$ is the effective electron-beam temperature 
which is given $[99,100]$ by

$$
T=T_{e}+\frac{m_{e}}{m_{i}} T_{i}
$$

Radiative recombination rate coefficients can be fitted by the expression $[101,102,103]$

$$
\alpha_{R R}=A \times\left[\sqrt{T / T_{0}}\left(1+\sqrt{T / T_{0}}\right)^{1-B}\left(1+\sqrt{T / T_{1}}\right)^{1+B}\right]^{-1}
$$

where $A, B, T_{0}$, and $T_{1}$ are parameters, depending on the ion species. Figure 3.20 shows how the $\mathrm{Fe}^{23+}$ radiative recombination coefficient changes with $T$. The black curve is from Eq. 3.21 and the red dashed curve is from Eq. 3.23. The parameters are from reference [103].

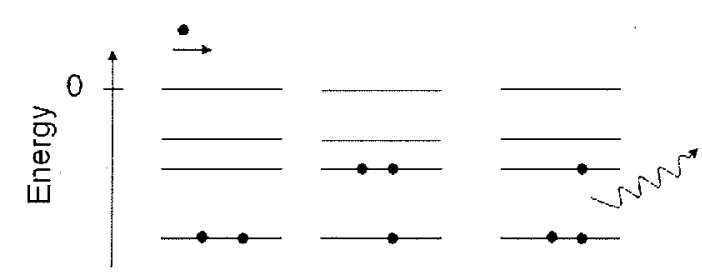

Dielectronic recombination

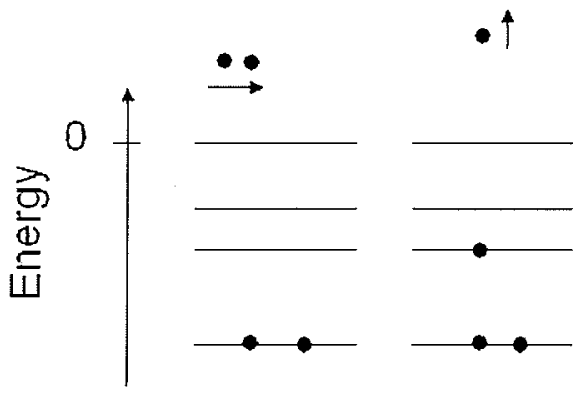

Three-body recombination

Figure 3.21: Dielectronic recombination. Figure 3.22: Three body recombination.

Figure 3.21 shows the dielectronic recombination (DR) process which occurs when an ion with at least one bound electron interacts with a free electron, forming a resonant state where the excess energy is transfered to the bound electron. This forms a short-lived doubly excited state which subsequently emits a photon, carrying 
away the excess energy. Dielectronic recombination is a two-step process; this process can be written as

$$
A^{q+}+e^{-} \rightarrow A^{(q-1)+* *} \rightarrow A^{(q-1)+*}+h \nu
$$

and the dielectronic recombination coefficient [104] is given by

$$
\alpha_{D R}=\frac{1}{(k T)^{3 / 2}} \sum_{j=1}^{4} c_{j} \exp \left(-\frac{E_{j}}{k T}\right)\left[\mathrm{cm}^{3} / s\right]
$$

where $c_{j}, E_{j}$ are parameters, depending on the type of ions.

Figure 3.22 shows the three-body recombination (TBR) process. This is a timereversed electron impact ionization, and it can occur when two electrons interact with an ion, one electron falls into a bound state, and the other carries away the excess energy. This process can be written as

$$
A^{q+}+e^{-}+e^{-} \rightarrow A^{(q-1)+}+e^{-} .
$$

The three body recombination coefficient [94] is given by (note that in this definition, $\alpha_{\mathrm{TBR}}$ contains one power of the electron density $\left.n_{e}\right)$

$$
\alpha_{T B R}=\left[2.0 \times 10^{-27} \mathrm{~cm}^{6} s^{-1}\right] Z^{3} n_{e}\left(\frac{e V}{k T}\right)^{4.5} .
$$

From Eq. 3.19 and Eq. 3.28, we can calculate the ion survival fraction $P$ during the electron cooling process. Figure 3.23 shows the $\mathrm{Fe}^{23+}$ survival fraction at a temperature of $4 \mathrm{~K}$. The electron cooling initial conditions are shown in Table 3.10. About $95.3 \% \mathrm{Fe}^{23+}$ can survive for $0.5 \mathrm{~s}$. Figure 3.24 shows the $\mathrm{Fe}^{23+}$ survival fraction 
Table 3.10: Initial conditions for the $\mathrm{Fe}^{23+}$ survival fraction at a temperature of $4 \mathrm{~K}$ or $300 \mathrm{~K}$.

\begin{tabular}{|l|l|}
\hline electron density $\left(\mathrm{cm}^{-3}\right)$ & $10^{7}$ \\
\hline electron number & $10^{7}$ \\
\hline number of $\mathrm{Fe}^{23+}$ & $10^{3}$ \\
\hline initial energy of $\mathrm{Fe}^{23+}(\mathrm{eV} / \mathrm{q})$ & 500 \\
\hline
\end{tabular}

at a temperature of $300 \mathrm{~K}$. About $97.6 \% \mathrm{Fe}^{23+}$ can survive for $0.8 \mathrm{~s}$.

$$
\frac{d P}{d t}=-P\left[\alpha_{R R}(T)+\alpha_{D R}(T)+\alpha_{T B R}(T)\right] n_{e}
$$

If we only consider radiative recombination at a temperature of $4 \mathrm{~K}$, the $\mathrm{Fe}^{23+}$

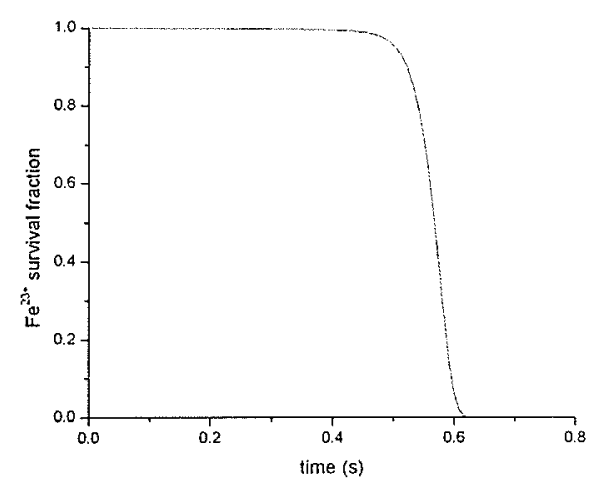

Figure 3.23: $\mathrm{Fe}^{23+}$ survival fraction at an environment temperature of $4 \mathrm{~K}$ including RR, DR, and TBR.

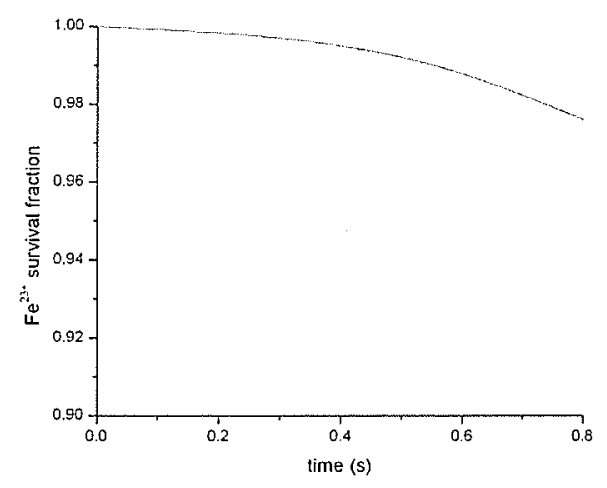

Figure 3.24: $\mathrm{Fe}^{23+}$ survival fraction at an environment temperature of $300 \mathrm{~K}$ including $R R, D R$, and TBR.

survival fraction curve is shown in Figure 3.25. About $95 \% \mathrm{Fe}^{23+}$ can survive for 0.8 s. Again only considering radiative recombination at a temperature of $300 \mathrm{~K}$, the 


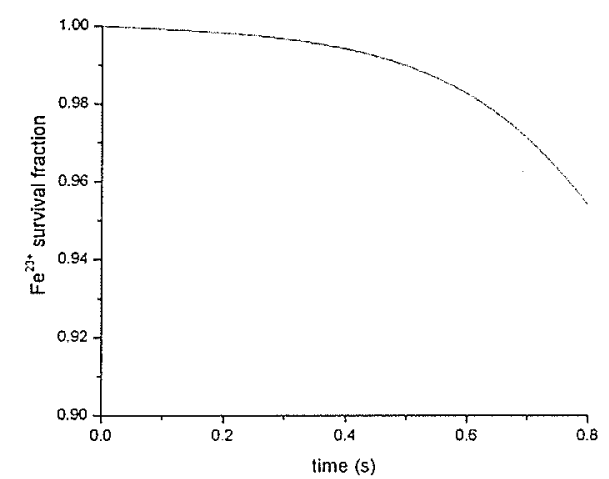

Figure 3.25: $\mathrm{Fe}^{23+}$ survival fraction at an Figure 3.26: $\mathrm{Fe}^{23+}$ survival fraction at environment temperature of $4 \mathrm{~K}$ including RR.

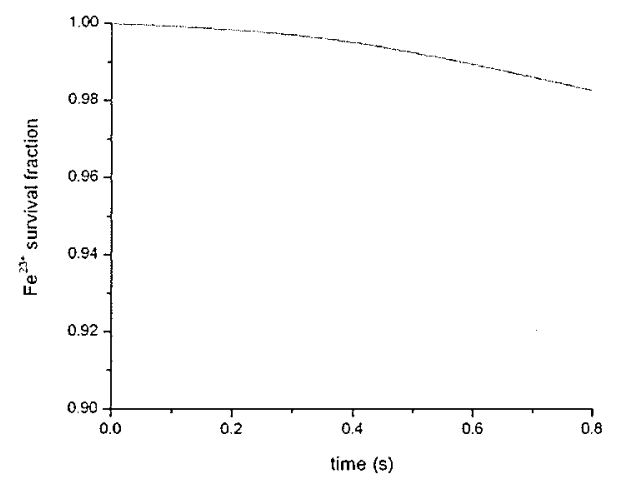

an environment temperature of $300 \mathrm{~K}$ including RR.

$\mathrm{Fe}^{23+}$ survival fraction curve is shown in Figure 3.26. About $98 \% \mathrm{Fe}^{23+}$ can survive for $0.8 \mathrm{~s}$.

To summarize, electron cooling and proton cooling of ions such as $\mathrm{C}^{6+}, \mathrm{Fe}^{23+}$, $\mathrm{Kr}^{36+}$, and $\mathrm{U}^{89+}$ have been modeled in the context of developing an ion cooler trap for the TITAN facility. For electron cooling of HCIs of initial energy of $500 \mathrm{eV} / q$, under the condition of $n_{e}=10^{7} \mathrm{~cm}^{-3}, N_{e}=10^{7}, N_{i}=10^{3}$, and an initial electron temperature of $300 \mathrm{~K}$, the cooling time is about $0.24 \mathrm{~s}-0.35 \mathrm{~s}$, depending on the ion species. Significant ion losses occur only after the HCIs are almost fully cooled. For proton cooling of HCIs of initial energy of $500 \mathrm{eV} / q$, under the condition of $n_{p}=10^{8} \mathrm{~cm}^{-3}$, $N_{p}=10^{8}, N_{i}=10^{3}$, and initial proton temperature of $1 \mathrm{eV}$, the cooling time is about 0.15 $\mathrm{s}-2.3 \mathrm{~s}$, depending on the ion species, comparable to the results for electron cooling. It remains to be seen whether the higher (compared to electron cooling) density of 
cold protons can be realized experimentally. As emphasized earlier, our calculations do not included the effect of the magnetic field on the collision dynamics and on the recombination processes. No analytic expressions are available for a scenario with a strong magnetic field. G. Zwicknagel et al. have developed simulations for the interaction of charged particles in a strong magnetic field, using computationally intensive particle-in-a-cell methods [89]. Their recently published results for electron cooling of bare uranium in a $6 \mathrm{~T}$ field within the context of the HITRAP experiment at GSI can be compared to our simple approach, and the result is shown in Figure 3.27 The cooling rate is found to be very similar for both approaches; within the expected accuracy of these models, we can consider the results to be essentially identical. This is a strong indication that the magnetic field might not play as an important role as has been speculated. Based on these findings, we have established a preliminary design for a cooler trap, accommodating electron and proton cooling for highly charged ions and buffer gas cooling for singly charged ions. The first goal will be to demonstrate the sympathetic cooling of HCIs to $\approx 1 \mathrm{eV} / q$ within about $1 \mathrm{~s}$. Ultimately, it might be possible to boost the coolant density significantly higher, using e.g. the rotating wall technique, where electron densities of $3 \times 10^{10} \mathrm{~cm}^{-3}$ have been achieved [105]. This could in turn lead to the cooling of HCIs in $100 \mathrm{~ms}$ or less. 


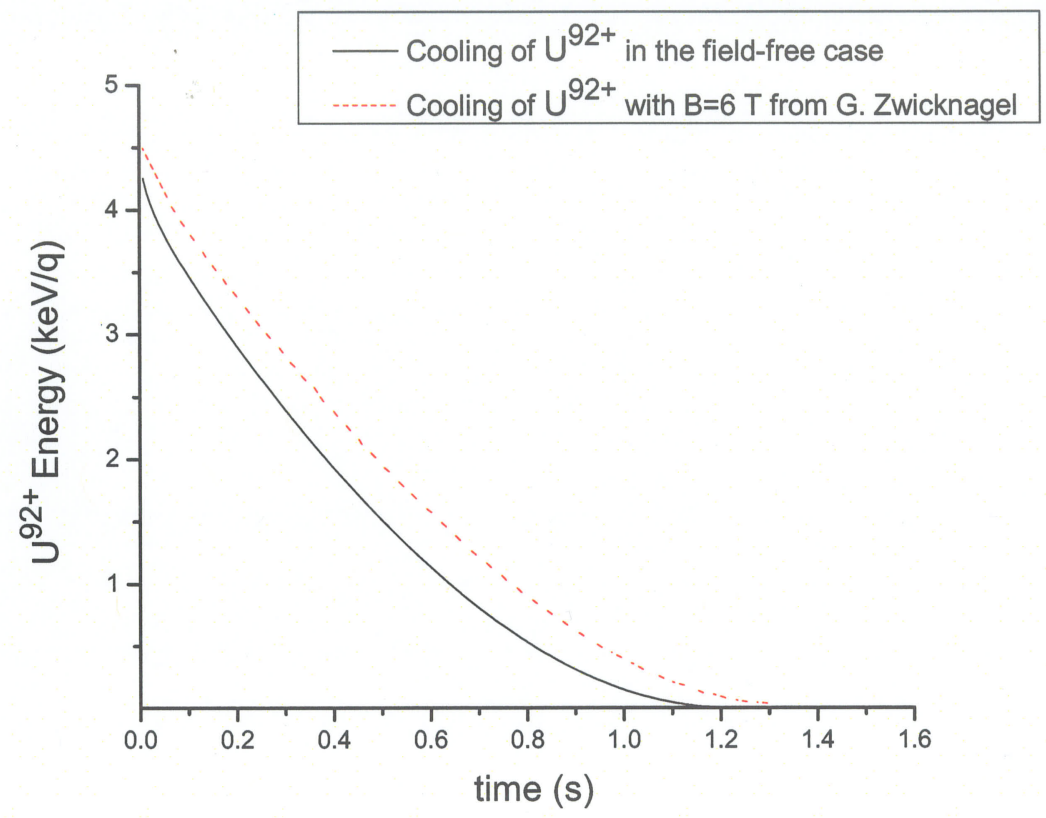

Figure 3.27: Cooling of $\mathrm{U}^{92+}$ in the field-free case and cooling of $\mathrm{U}^{92+}$ with $\mathrm{B}=6 \mathrm{~T}$ from G. Zwicknagel. 


\section{Chapter 4}

\section{DESIGN OF THE COOLER TRAP}

Based on the discussions of various cooling mechanisms presented in the previous chapter, it was decided that the most promising route would be sympathetic cooling via the Coulomb interaction, using either self-cooling electrons or cold protons. Sympathetic cooling has proven to be an efficient, widely applicable method, and electron cooling of protons was demonstrated and has been extensively used in the efforts to produce anti-hydrogen [106]. At RIKEN (Japan) an effort is underway to cool highly charged ions with positrons and electrons [107], and the HITRAP collaboration at GSI (Germany) is currently readying an electron cooler trap [108]. The cooling times involved and the required particle densities clearly require an additional trap for this purpose. Electron cooling relies on synchrotron radiation in a strong magnetic field of several Tesla, demanding the use of a Penning trap. In a generic Penning trap, particles of opposite charge cannot be stored simultaneously. However, a potential well scheme known as a nested trap can be employed and will be discussed below. 


\subsection{Schematic diagram for cooling of HCIs}

For proton cooling, protons should be trapped in a region such as the harmonic potential region shown in Figure 4.1a, with the potential at both ends of the trapped proton region higher than the potential inside the trapped proton region. An axial electric field force directed to the center of this region traps the protons in the axial direction. Because of their high energies, highly charged ions need the higher potential well shown in Figure 4.1b. When highly charged ions cross the trapped proton region, they collide with protons in the region, and proton cooling can occur. The cooled highly charged ions are trapped in the small proton trapping region shown in Figure 4.1c. By lowering the potential as shown in Figure 4.1d, low-charge protons are ejected first.

a
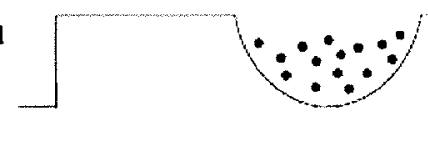

c

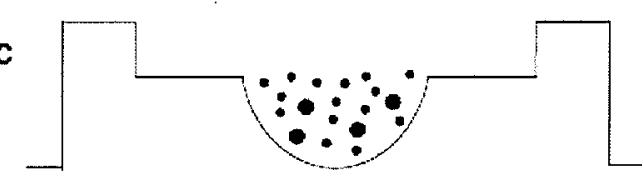

b
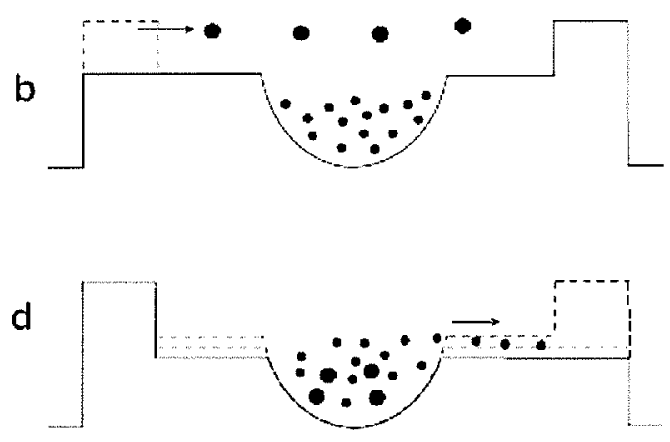

Figure 4.1: Schematic diagram for proton cooling of HCIs. Larger filled circles represent HCI; smaller filled circles represent protons. a) Protons are prepared in a harmonic trap after multiple injections; b) highly charged ions are injected into a larger square-well trap where coolant protons are contained in the harmonic trap. c) HCIs are cooled down in collisions with protons; d) HCIs may be further cooled by evaporative cooling of protons during a slow ejection of protons. 
For electron cooling, electrons should be trapped in a region such as the harmonic potential region shown in Figure 4.2a, where the potential at both ends of the electron trapping region is lower than the potential inside the electron trapping region. An axial electric field force directed to the center of this region traps electrons in the axial direction. Highly charged ions need the high potential well shown in Figure $4.2 \mathrm{~b}$, similar to the proton cooling case. When highly charged ions cross the electron trapping region, electrons collide with ions, electron cooling can occur, and highly charged ions finally cool down shown in Figure 4.2c. By lowering the potential as shown in Figure 4.2d, cooled highly charged ions are ejected.

a

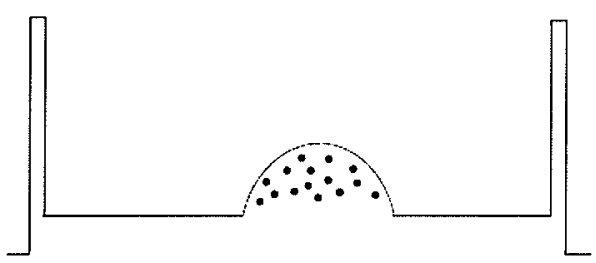

c

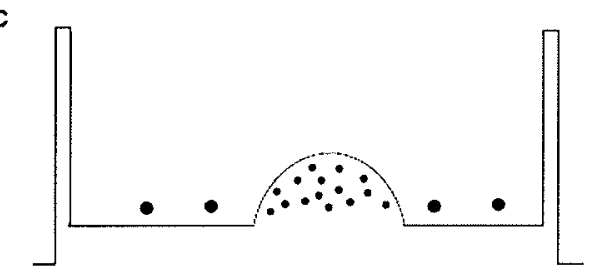

b i

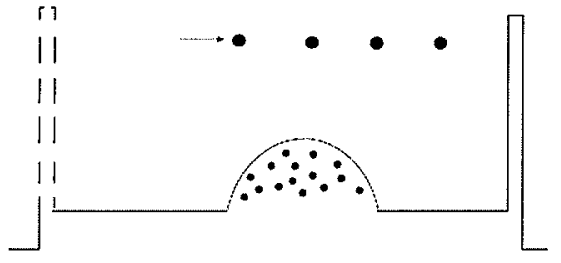

d

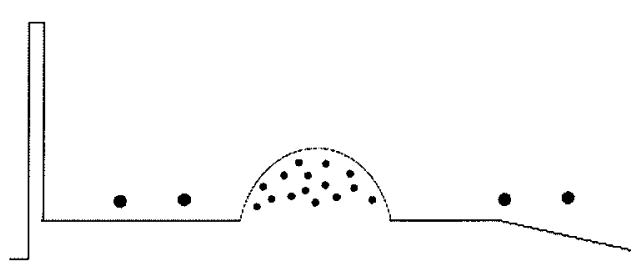

Figure 4.2: Schematic diagram for electron cooling of HCIs. Larger filled circles represent HCI; smaller filled circles represent electrons. a) Electrons are prepared in a harmonic trap after injection; b) highly charged ions are injected into a larger square-well trap where coolant electrons are contained in the harmonic trap. c) HCIs are cooled down in collisions with electrons; d) HCIs are ejected. 


\subsection{Designing potentials}

Before we design a cooler trap, we use SIMION 7.0, which is a commercial software package, to calculate the potential for certain geometries of the cooler trap and try to find how to generate the potential we need. We use SIMION to simulate the ions' trajectories when they enter the magnetic field, electric field, or both, and find the correct parameters for geometry of the injection system, voltages at the different electrodes, and so on.

We simulate the ions' path in the electric field. To do this, we first need to define the electrode geometry in a three-dimensional region. The three-dimensional volume is subdivided into a grid with grid spacing $h$ in three dimensions. The volume then consists of many grid points $(i, j, k)$. The potential $V$ can be solved by Laplace's Equation

$$
\nabla^{2} V(i, j, k)=\frac{\partial^{2} V(i, j, k)}{\partial^{2} x}+\frac{\partial^{2} V(i, j, k)}{\partial^{2} y}+\frac{\partial^{2} V(i, j, k)}{\partial^{2} z}=0
$$

where the free charge is zero in that region. We use the following approximate relations:

$$
\begin{aligned}
& \frac{\partial^{2} V(i, j, k)}{\partial^{2} x} \approx \frac{V_{i-1, j, k}+V_{i+1, j, k}-2 V_{i, j, k}}{h^{2}} \\
& \frac{\partial^{2} V(i, j, k)}{\partial^{2} y} \approx \frac{V_{i, j-1, k}+V_{i, j+1, k}-2 V_{i, j, k}}{h^{2}} \\
& \frac{\partial^{2} V(i, j, k)}{\partial^{2} z} \approx \frac{V_{i, j, k-1}+V_{i, j, k+1}-2 V_{i, j, k}}{h^{2}} .
\end{aligned}
$$


Inserting the Eq. 4.2, Eq. 4.3, and Eq. 4.4 into Eq. 4.1, we have

$$
\frac{V_{i-1, j, k}+V_{i+1, j, k}-2 V_{i, j, k}}{h^{2}}+\frac{V_{i, j-1, k}+V_{i, j+1, k}-2 V_{i, j, k}}{h^{2}}+\frac{V_{i, j, k-1}+V_{i, j, k+1}-2 V_{i, j, k}}{h^{2}}=0
$$

From Eq. 4.5,

$$
V_{i, j, k}=\frac{V_{i-1, j, k}+V_{i+1, j, k}+V_{i, j-1, k}+V_{i, j+1, k}+V_{i, j, k-1}+V_{i, j, k+1}}{6} .
$$

Eq. 4.6 is the finite-difference approximation, i.e. the potential of a grid point is the average value of its nearest six neighbors. SIMION puts the electrode geometry into the three dimensional volume, and the potentials of the electrode grid points are held fixed. The potentials of non-electrode points can then be adjusted according to Eq. 4.6 to get the correct potential values for the non-electrode points. To do this, a group of arbitrary potential values $V_{i, j, k}$ are first given to all the non-electrode points in the volume. Initially, they will not satisfy Eq. 4.6; that is to say

$$
V_{i, j, k} \neq \frac{V_{i-1, j, k}+V_{i+1, j, k}+V_{i, j-1, k}+V_{i, j+1, k}+V_{i, j, k-1}+V_{i, j, k+1}}{6}
$$

Next, each old value $V_{i, j, k}$ is replaced with a new value $V_{i, j, k}^{\prime}$, given by

$$
V_{i, j, k}^{\prime}=\frac{V_{i-1, j, k}+V_{i+1, j, k}+V_{i, j-1, k}+V_{i, j+1, k}+V_{i, j, k-1}+V_{i, j, k+1}}{6}
$$

Moving from one grid point to the next, the values for all grid points are adjusted in this way, leaving the electrode potentials fixed. The process is then repeated, until it finally converges on a set of values which satisfy the finite-difference approximation for all the grid points. This method is called the relaxation method. 
SIMION makes use of a geometry file to mark the grid points and to define the electrode geometry. We save this file as *.PA\#, where $*$ can be any name. From *.PA\#, by the relaxation method, the potentials for all the grid points are calculated according to the electrode boundary conditions. SIMION adjusts all the potential values once according to the finite-difference approximation; that is, a single iteration is first performed. If the potential value for the grid points changes by less than the convergence objective (minimum $1.0 \times 10^{-7}$ ) between successive iterations, the potential refining is finished, otherwise the process is repeated. Once converged, some specific SIMION files are generated. For instance, for a system with $N$ electrodes, after refining from *.PA\#, SIMION generates $N+1$ specific potential solution files *.PA0, *.PA1, ...., *.PA9, *.PAa, *.PAb, .....*.PA1 is a refined array; it is the specific finite-difference approximation solution when the voltage at electrode \#1 is set at $10 \mathrm{kV}$ and the voltages are set to zero at the other electrodes. *.PA2 is also a refined array; it is the specific finite-difference approximation solution when the voltage at electrode $\# 2$ is set to $10 \mathrm{kV}$ and the voltages are set to zero at all other electrodes, and so on for all the electrodes. The solutions are independent of the real voltages at the electrodes. Real voltages at all the electrodes can be set by the "Fast Adjust" button or by a user program where the variables Adj_Elect00 to Adj_Elec30 are set to the real potentials of the different electrodes. After a "Fast Adjust", the fast adjust file *.PAO is changed and will supply the potentials for all the grid points 
which are given by

$$
* . P A_{0}(i, j, k)=\frac{V 1}{100000} * . P A 1(i, j, k)+\frac{V 2}{100000} * . P A 2(i, j, k)+\ldots \ldots
$$

where $V 1, V 2, \ldots \ldots$ are respectively the voltages $(\mathrm{V})$ at the electrodes.

The potential along the central line is very important, as it supplies the axial trap well. As an example of a simple geometry, a cylindrical Penning trap is shown in Figure 4.3; there are 11 equal-length electrodes, arranged according to reference [109]. The length of each electrode is set to $7 \mathrm{~mm}$, and the radius of the electrodes is set to $10 \mathrm{~mm}$. Because the geometry in SIMION is given according to grid points, the ratio of radius to length of each electrode is set to about 1.42 ; the gap is $0.3 \mathrm{~mm}$. Laplace's equation solutions can be solved analytically to generate the axial harmonic potential. Table 4.1 gives the cylindrical trap geometry and the electrode voltages [109]. $V_{0}$ is the potential difference between the endcap electrode and the ring electrode; the electrode definition is shown in Figure 2.10. This cylindrical trap geometry was loaded into SIMION and the voltages $V_{c a l c}$ at different electrodes are given in Table 4.2. These voltages satisfy the conditions in Table 4.1 , where $V_{0}$ is equal to $20 \mathrm{~V}$. After potential refining and "Fast Adjust", the axial potential can be obtained from the potential array, .PA0, and is shown by the black curve in Figure 4.4 , which is a harmonic potential in that region as expected.

As a check, we can make use of *.PA1,*.PA2, ...., *.PAa, *.PAb, .... and the target potential $T(i, j, k)$ which is required for a trap to know the voltages $V 1, V 2$, 
so that this group of voltages gives the potential $T(i, j, k)$.

$$
\frac{V 1}{100000} * . P A 1(i, j, k)+\frac{V 2}{100000} * . P A 2(i, j, k)+\ldots . .=T(i, j, k)
$$

As an example, we load same geometry as above into SIMION. After refining, the potential arrays *.PA1, *.PA2, ...., *.PAa, and *.PAb are generated; there are 11 potential arrays which are independent of real voltage at those cylindrical electrodes, and we take the axial potential shown by the black curve in Figure 4.4 as the target potential, and work out the voltages at the cylindrical electrodes. We define the following function to find the voltages at different electrodes:

$$
\begin{array}{r}
G=\sum_{i}\left[\frac{V_{1}}{100000} * . P A 1\left(i, j_{0}, k_{0}\right)+\frac{V_{2}}{100000} * . P A 2\left(i, j_{0}, k_{0}\right)+\ldots \ldots+\right. \\
\left.\frac{V_{11}}{100000} * \cdot P A b\left(i, j_{0}, k_{0}\right)-T\left(i, j_{0}, k_{0}\right)\right]^{2},
\end{array}
$$

$i$ is summed in the target function region where we hope that the potential is near the target potential when voltages $V_{1}, V_{2}, \ldots \ldots$, and $V_{11}$ are applied at the 11 electrodes. $T\left(i, j_{0}, k_{0}\right)$ is the target potential along the axial direction, the axial line is in the $x$ direction, and $j_{0}$ and $k_{0}$ are constants.

The function $G$ shows the difference between the potential calculated by SIMION and the target potential in the special axial position region. If two potentials are very nearly the same, that means $G$ has a minimum, so we have

$$
\begin{array}{r}
\frac{\partial G}{\partial V_{m}}=0 . \\
m=1,2, \ldots \ldots, 11
\end{array}
$$


From the set of Equations 4.12, normally we can get 11 variables $V_{1}, V_{2}, \ldots .$. , and $V_{11}$. The same method can be applied to other geometries too.

Considering $V_{1}=V_{2}=V_{3}=V_{9}=V_{10}=V_{11}=-V_{6}, V_{4}=V_{8}$, and $V_{5}=V_{7}$, Eqns. 4.12 reduce to only three equations; there are three independent variables. We also simplify the target function. If we fit the potential curve shown in black in Figure 4.4 , it will give a target potential at the two ends that is simply a constant in a small axial region; it is a harmonic function in the central axial region. Let $G$ have a minimum in these three regions; the voltages $V_{\text {Sim }}$ are then found and are shown in Table 4.2. The red dashed curve in Figure 4.4 is the axial potential which is generated by applying these electrode voltages. The very good agreement between the two curves demonstrates the validity of the numerical method using SIMION.

$\begin{array}{lllllllllllllllll}1 & 2 & 4 & 5 & 6 & 7 & 5 & 9 & 10 & 11\end{array}$

Figure 4.3: Cylindrical trap.

\subsection{Injection of highly charged ions, electrons and protons}

Because the injection system is very important to the cooler trap, we use SIMION to simulate the injection of highly charged ions, electrons and protons. For the 7 Tesla magnetic field, we take data from the manufacturer of our magnet, Cryomagnetics, 
Table 4.1: Geometry of the cylindrical Penning trap and electrode voltages.

\begin{tabular}{|l|l|}
\hline Radius/length of electrode & 1.42 \\
\hline$V_{1} / V_{0}$ & -0.3856 \\
\hline$V_{2} / V_{0}$ & 0.4830 \\
\hline
\end{tabular}

Table 4.2: $V_{\text {calc }}$ : Voltages at the cylindrical electrodes from the analytical approach. $\mathrm{V}_{\text {Sim }}$ : Voltages at the cylindrical electrodes found by our numerical method using SIMION.

\begin{tabular}{|l|l|l|}
\hline Electrode \# & $V_{\text {Calc }}$ & $V_{\text {Sim }}$ \\
\hline 1 & 10 & 9.9888 \\
\hline 2 & 10 & 9.9888 \\
\hline 3 & 10 & 9.9888 \\
\hline 4 & 9.66 & 9.535 \\
\hline 5 & -7.712 & -7.6969 \\
\hline 6 & -10 & -9.9888 \\
\hline 7 & -7.712 & -7.6969 \\
\hline 8 & 9.66 & 9.535 \\
\hline 9 & 10 & 9.9888 \\
\hline 10 & 10 & 9.9888 \\
\hline 11 & 10 & 9.9888 \\
\hline
\end{tabular}




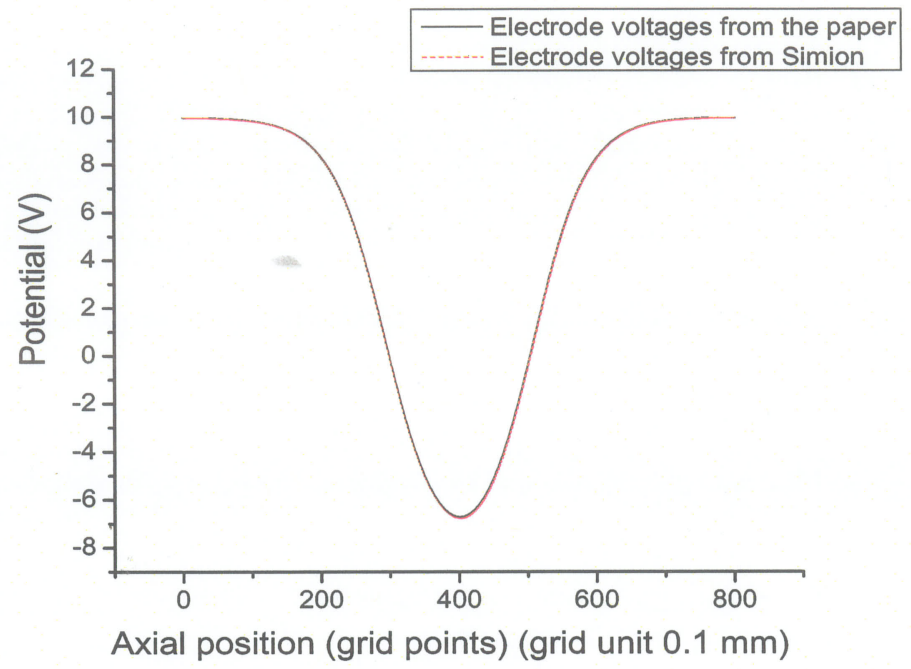

Figure 4.4: Cylindrical trap axial potential; the two curves are overlapped.

Inc., and we simulate trajectories of highly charged ions, electrons and protons in the electric field and the magnetic field. We try to find a set of parameters, such as the voltages at different electrodes, which make the transportation efficiency high, and result in final energies of highly charged ions, electrons and protons which are suitable initial conditions for the cooler.

\subsubsection{Magnetic field}

Normally, we can use the user program to load magnetic field data into SIMION. Appendix D shows how to load a magnetic field; the file 7teslamagneticfield.dat is the data file supplied by the maker of the magnet for the cooler trap. It is a two column data file; the Penning trap magnet has cylindrical symmetry, so the magnetic 
field location is specified by the two variables of axial and radial position, and the magnetic field vector is given as axial and radial components. The first column in the file is the axial magnetic field, and the second column is the radial magnetic field. The data in the first row is for grid point $(0,0)$ which is at the center of the magnet, the first variable being axial position and second one being the radial position. The data in subsequent rows go according to $(0,0),(1,0), \ldots \ldots,(0,1),(1,1), \ldots \ldots$. In SIMION, when an ion's position is not at the grid point, its magnetic field at that position will be given according to the 4 nearest grid points' magnetic fields. The 4 nearest grid points are chosen on the same radial surface as the ion position.

\subsubsection{Simulation of charged particle injection}

Magnetic fields are important to the TITAN project. The electron beam ion trap, cooler trap, and Penning trap all use magnetic fields to trap charged particles in the radial direction. Because of magnetic field gradients, charged particles experience an axial force; this makes it difficult for charged particles to enter a strong magnetic field region from a weak magnetic field region. Proper parameters must be chosen so that ions or protons or electrons can be injected into the trap in the strong magnetic field region. In the field, Newton's equation is given by

$$
\begin{aligned}
\frac{d v_{x}}{d t} & =a_{x}\left(x, y, z, v_{x}, v_{y}, v_{z}, t\right) \\
\frac{d v_{y}}{d t} & =a_{y}\left(x, y, z, v_{x}, v_{y}, v_{z}, t\right) \\
\frac{d v_{z}}{d t} & =a_{z}\left(x, y, z, v_{x}, v_{y}, v_{z}, t\right)
\end{aligned}
$$




$$
\begin{aligned}
& \frac{d x}{d t}=v_{x} \\
& \frac{d y}{d t}=v_{y} \\
& \frac{d z}{d t}=v_{z}
\end{aligned}
$$

where $a$ is the acceleration given by the electric and magnetic forces and interactions e.g. with a buffer gas.

The velocity $\left(v_{x}, v_{y}, v_{z}\right)$ and positions $(x, y, z)$ along the three directions are determined by the equations above. When we load the magnetic field data file and .PA0 into SIMION, we calculate the force on the ion, therefore the acceleration is known. Furthermore, from Eq. 4.13, the ion trajectory can be calculated by the fourth-order Runge-Kutta method [110], step by step from a given starting position.

\section{Simulation of proton injection}

Proton injection is shown in Figure 4.5. The axial magnetic field can be seen in Figure 4.6, with the magnetic field stronger on the left side. The injection system consists of the trap, drift tube, and lens system. Figure 4.7 shows the enlarged trap region. The geometry file is shown in Appendix E; the trap consists of 14 cylindrical electrodes, each of them is $19 \mathrm{~mm}$ in radius and $12.7 \mathrm{~mm}$ in length, with $2 \mathrm{~mm}$ space between electrodes. In this setup, the first trap electrode is $1 \mathrm{~mm}$ from the magnetic field center. The drift tube is $343 \mathrm{~mm}$ long and its radius is $19 \mathrm{~mm}$. The center of the drift tube is $382 \mathrm{~mm}$ from the center of the magnetic field. The two lenses have similar structure, each consisting of three electrodes. The electrodes of the first lens, which is 
nearest to the trap region, are $100 \mathrm{~mm}$ long with a radius of $60 \mathrm{~mm}$. The gap between electrodes is $20 \mathrm{~mm}$, and the end point of first lens is $1241 \mathrm{~mm}$ from the magnetic field center. The electrodes of the second lens are $60 \mathrm{~mm}$ long with a radius of 40 $\mathrm{mm}$. The gap between them is $10 \mathrm{~mm}$, and the end point of the second lens is 2241 $\mathrm{mm}$ from the magnetic field center. In Figure 4.5, protons enter into the electric and magnetic fields from a position $2400 \mathrm{~mm}$ from the magnetic field center. The voltages at the trap electrodes are all set to $0 \mathrm{~V}$. Voltages for the two end electrodes of the first lens are set to zero, the voltage for the middle electrode of the first lens is set to $-5754 \mathrm{~V}$, the voltages for two end electrodes of the second lens are set to zero, and the voltage for the middle electrode of the second lens is set to $-3754 \mathrm{~V}$. The voltage of the drift tube is set to $0 \mathrm{~V}$ at the beginning, but when the protons pass its center, the voltage is switched to $-2000 \mathrm{~V}$. If not, the proton energy will not be reduced to the very low desired energy. Protons entering the trap are chosen randomly from an angle-radius ellipse with a maximum angle of $0.5^{\circ}$ with the axis, and a radius of 4 $\mathrm{mm}$. The initial proton energy is $2.14 \mathrm{keV}$. We find that if the protons end up with a final kinetic energy of about $139 \mathrm{eV}$ in the trap region, as in the simulation shown in Figure 4.5 , the injection efficiency is $100 \%$. However, this proton energy is too high for our purposes.

If the proper parameters are chosen, the proton energy can be reduced to a few $\mathrm{eV}$. Figure 4.8 and 4.9 show how this can be done. In Figure 4.8, the voltage for the middle electrode of the first lens is $-6000 \mathrm{~V}$, the voltage for the middle electrode of 


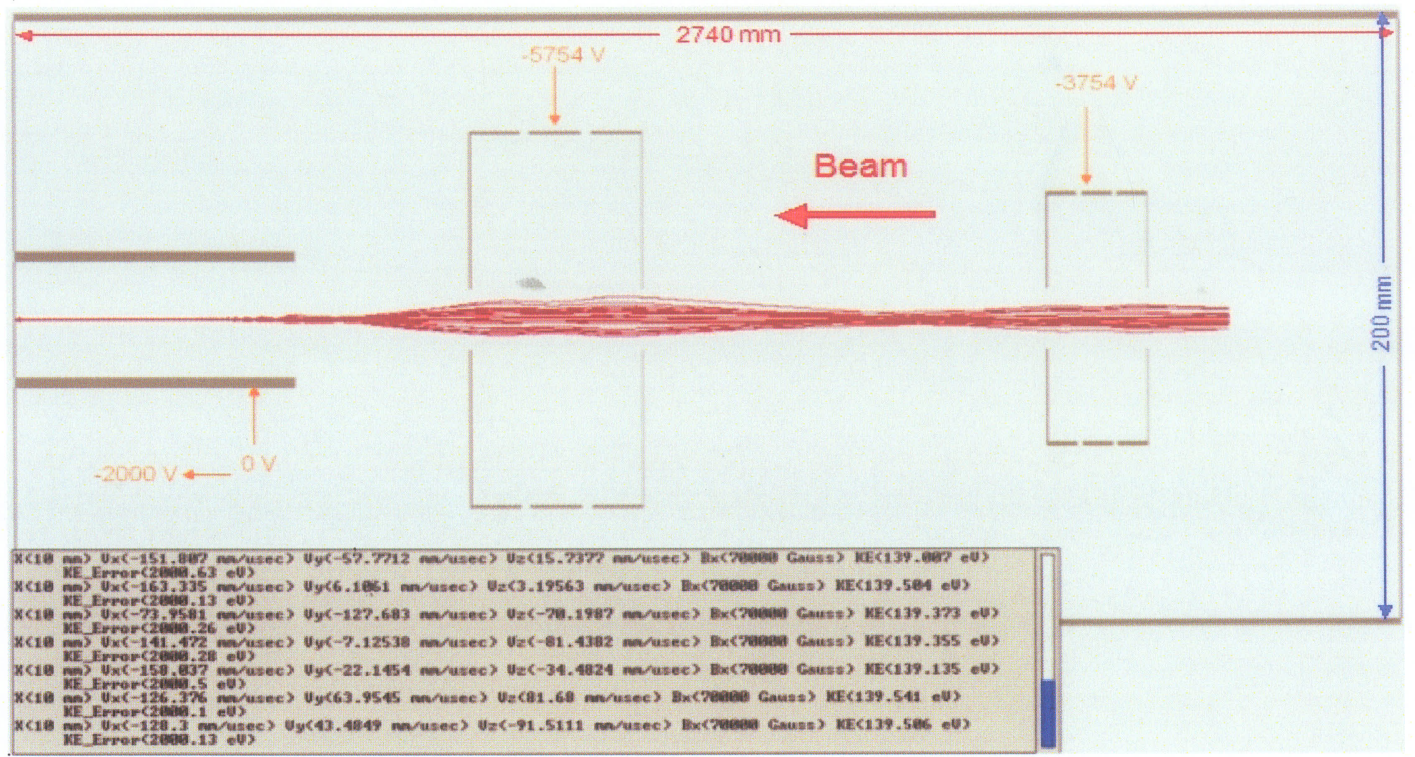

Figure 4.5: Proton injection, starting at a position $2400 \mathrm{~mm}$ from the center of the magnetic field (space-charge effects are not included). In this and the following SIMION screenshots, the numerical information in the text window is irrelevant.

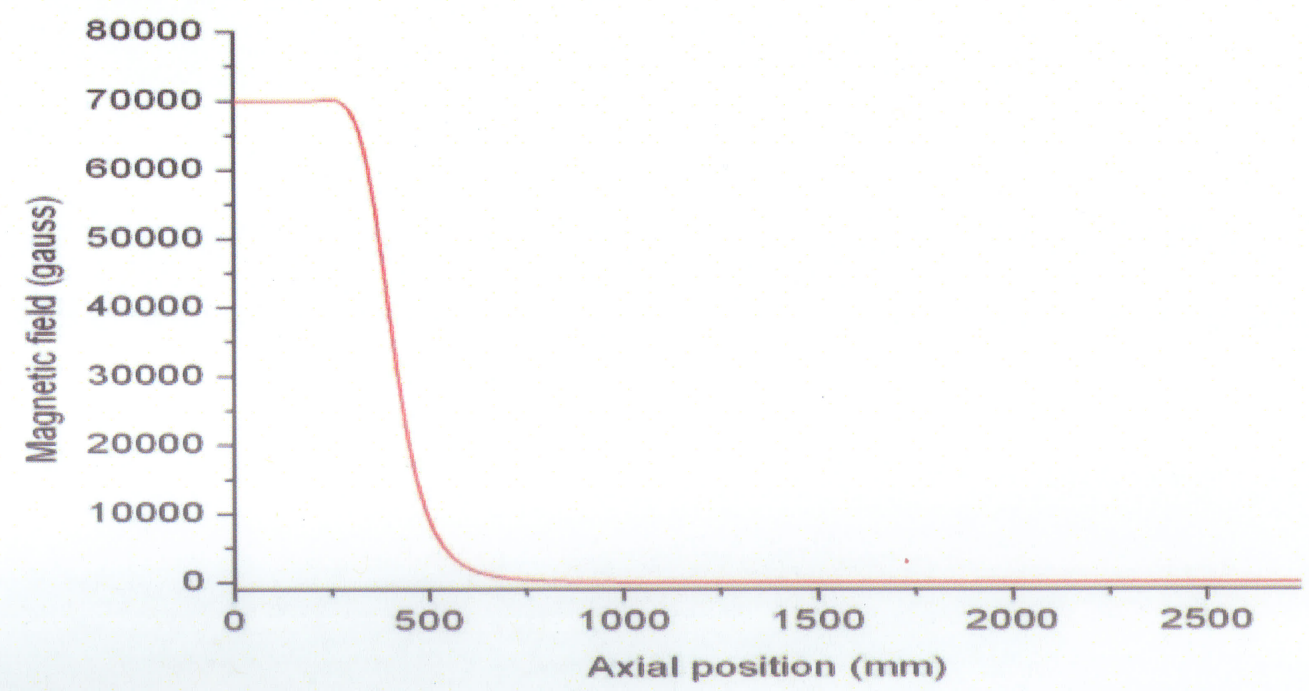

Figure 4.6: Axial magnetic field curve for our 7 Tesla magnet. 
DF-

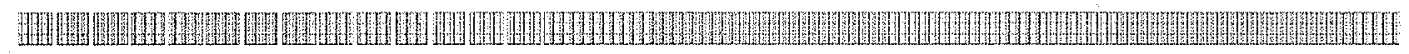

Figure 4.7: Enlarged trap region.

second lens is $-3100 \mathrm{~V}$, the proton starting position is $4 \mathrm{~mm}$ from the central line and the angle between the proton velocity direction and the central line is $0.5^{\circ}$. In Figure 4.9 , the voltage for the middle electrode of the first lens is $-5508 \mathrm{~V}$, the voltage for the middle electrode of the second lens is $-4408 \mathrm{~V}$, the proton starting position is $0 \mathrm{~mm}$ from the central line and the angle between the proton velocity direction and the central line is $0.5^{\circ}$. For a single proton, by only adjusting some parameters, that proton can be injected into the trap region with low energy.

Figure 4.10 shows protons injected into the trap region with very low final energy $(16 \mathrm{eV})$ from a position $1789 \mathrm{~mm}$ from the center of the magnetic field. Because of the axial force, some protons are reflected back. The voltage for the middle electrode of the first lens is $-3900 \mathrm{~V}$, others are zero; the initial proton energy is $2.016 \mathrm{keV}$, and, in a way similar to Figure 4.5, protons are injected with random initial radius and random initial angle. The maximum radius is still $4 \mathrm{~mm}$, but the maximum angle 


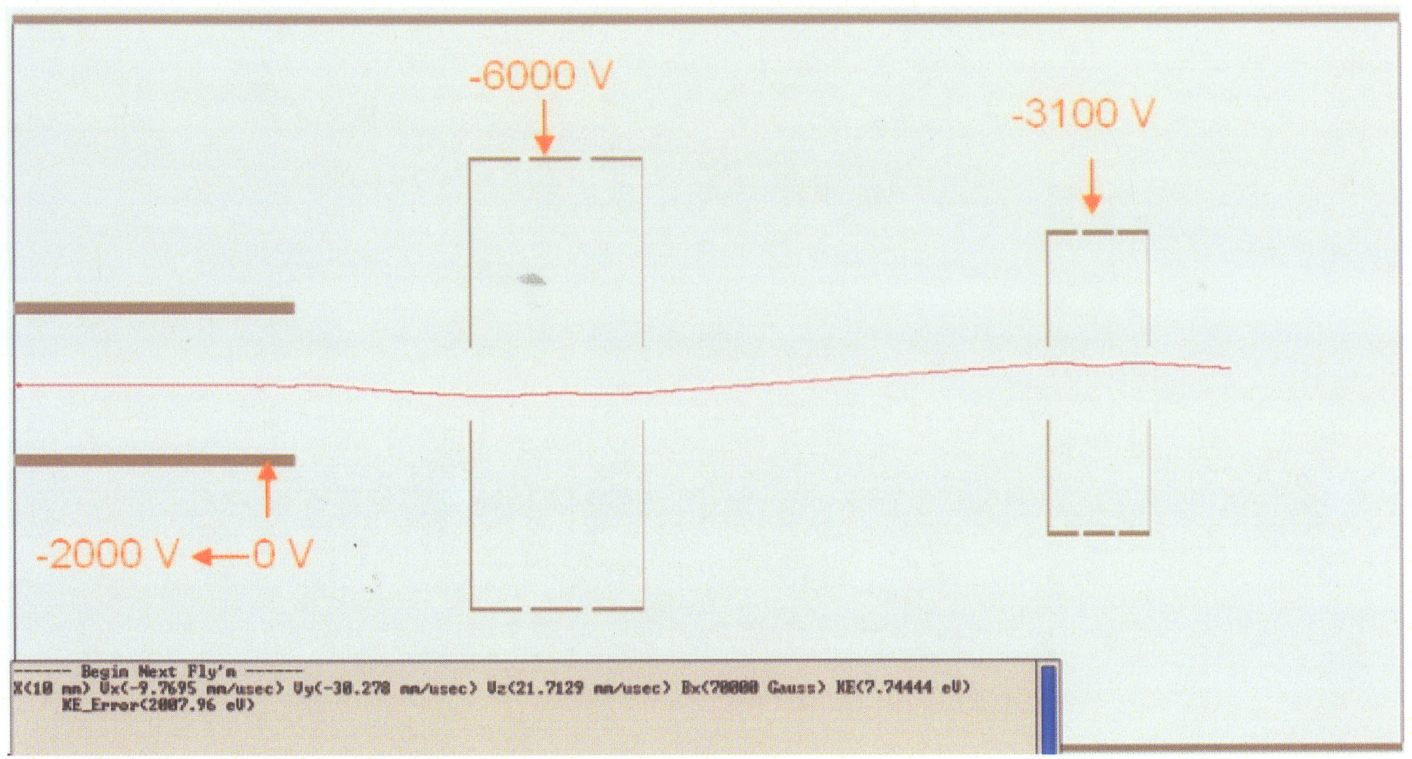

Figure 4.8: Single proton injection with an initial proton position $4 \mathrm{~mm}$ from the central line.

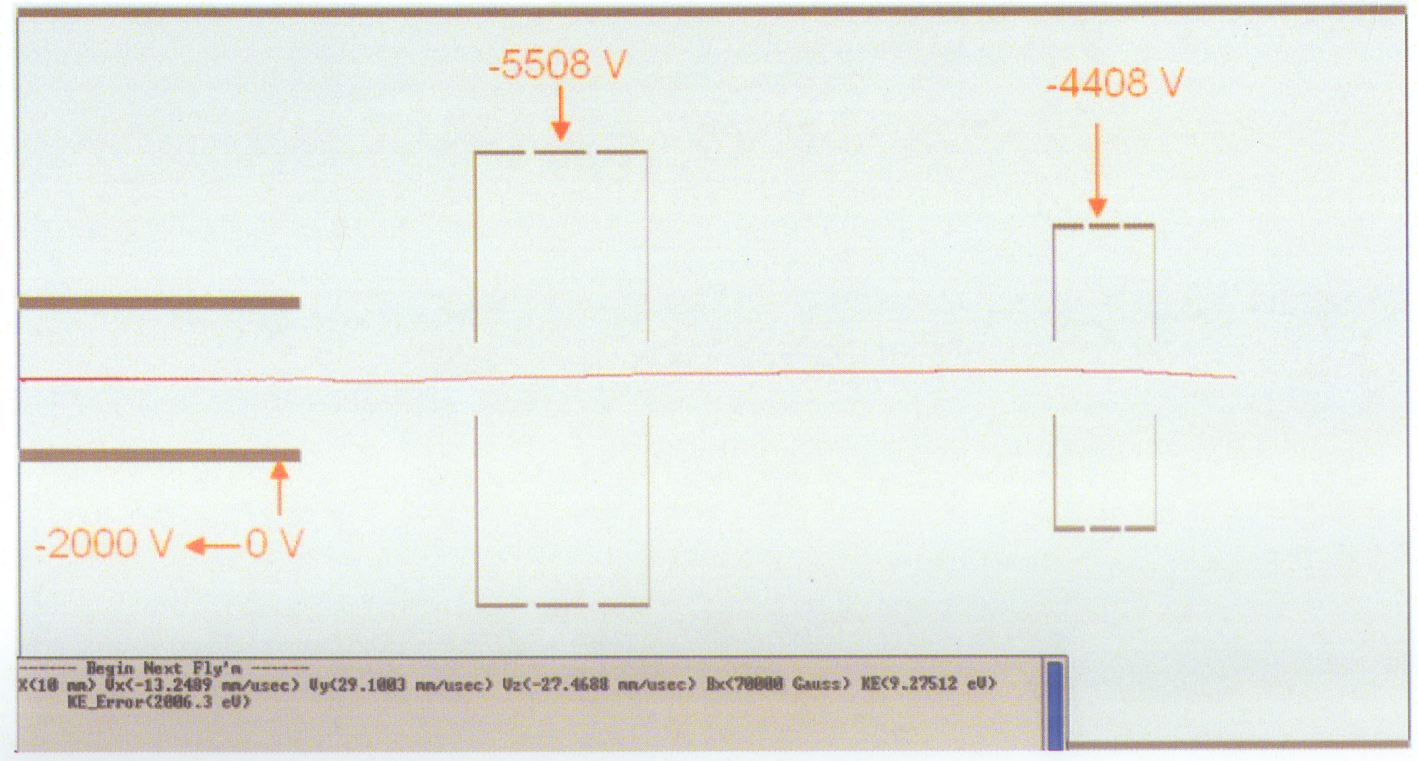

Figure 4.9: Single proton injection with an initial proton position $0 \mathrm{~mm}$ from the central line. 
is reduced to $0.1^{\circ}$. For 200 protons injected, more than $80 \%$ of them reach the trap region. For increasing angle, the beam emittance is large, and more protons will be reflected back.

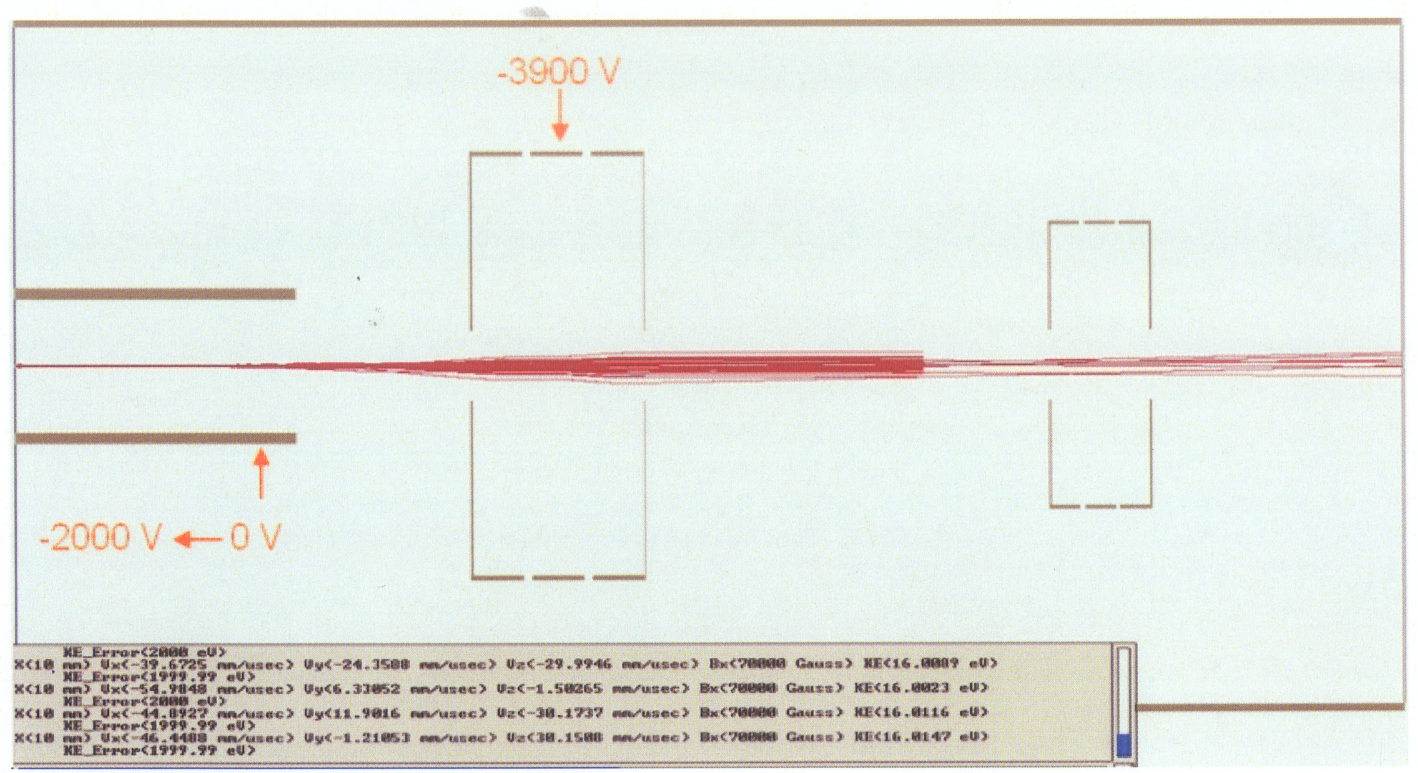

Figure 4.10: Proton injection, starting at a position $1789 \mathrm{~mm}$ from the center of the magnetic field.

If protons are injected from a relatively distant position such as shown in Figure 4.11, where protons are injected from a starting position $2600 \mathrm{~mm}$ from the magnetic field center, the final proton energies are about $16 \mathrm{eV}$. If 200 protons are injected, about $50 \%$ can reach the trap region. For this simulation, the lens system has been moved $200 \mathrm{~mm}$ further from the center of the magnetic field compared to the position shown in Figure 4.5. The initial maximum radius is $4 \mathrm{~mm}$, the initial maximum angle is $0.5^{\circ}$, the voltage of middle electrode of the first lens is $-3900 \mathrm{~V}$, 
the voltage of middle electrode of the second lens is $-3300 \mathrm{~V}$, and the initial proton energy is $2.016 \mathrm{keV}$.

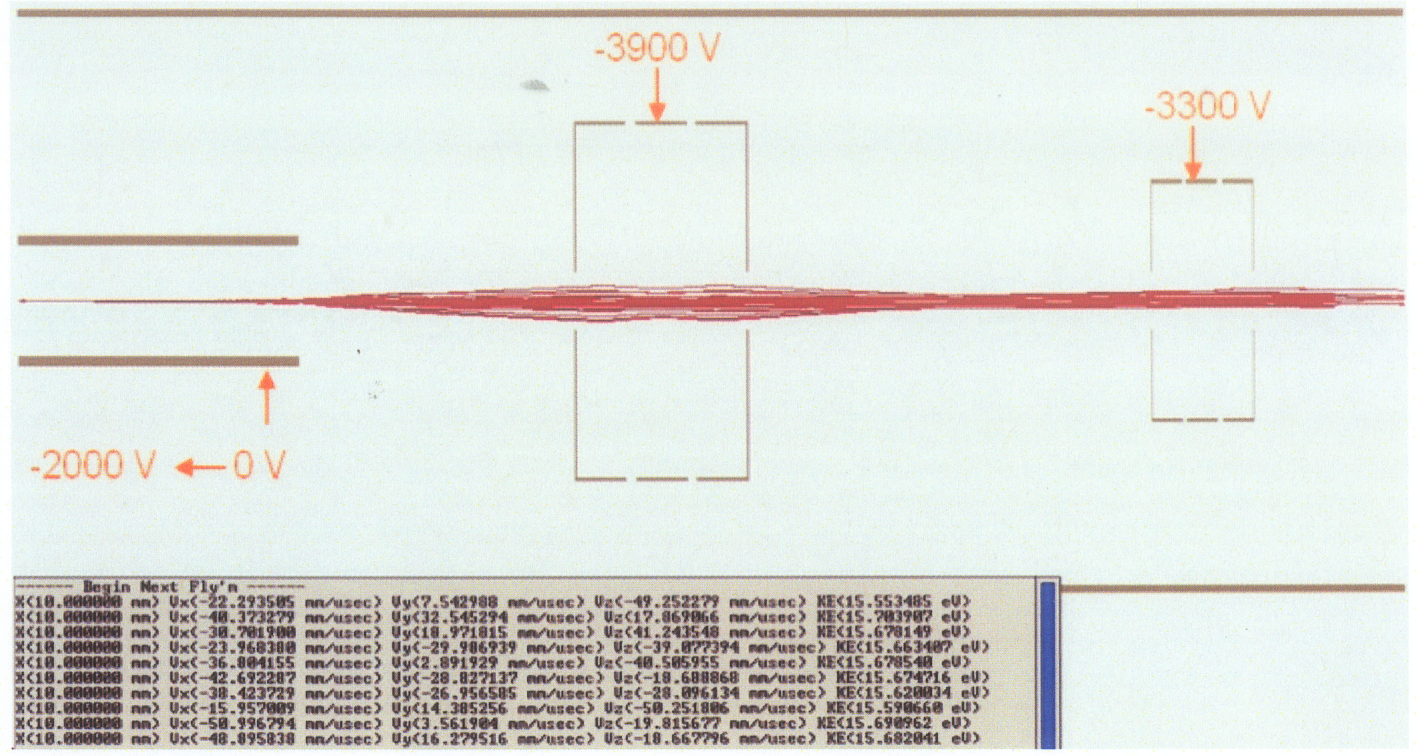

Figure 4.11: Proton injection, starting at a position $2600 \mathrm{~mm}$ from the center of the magnetic field.

Simulation of highly charged ion injection

Figure 4.12 shows $\mathrm{Kr}^{36+}$ injection. The geometry is the same as for the proton injection shown in Figure 4.5. The initial maximum radius is $4 \mathrm{~mm}$, the initial maximum angle is $0.5^{\circ}$, the voltage of the middle electrode of the first lens is $-5754 \mathrm{~V}$, the voltage of the middle electrode of the second lens is $-3754 \mathrm{~V}$, and the $\mathrm{Kr}^{36+}$ energy is 2.128 $\mathrm{keV} / q$. We find that the $\mathrm{Kr}^{36+}$ energy can also be reduced to a few hundred $\mathrm{eV} / q$. Ions with a few hundred $\mathrm{eV} / q$ are suitable for electron cooling and proton cooling as 
discussed in section 3.1.5.

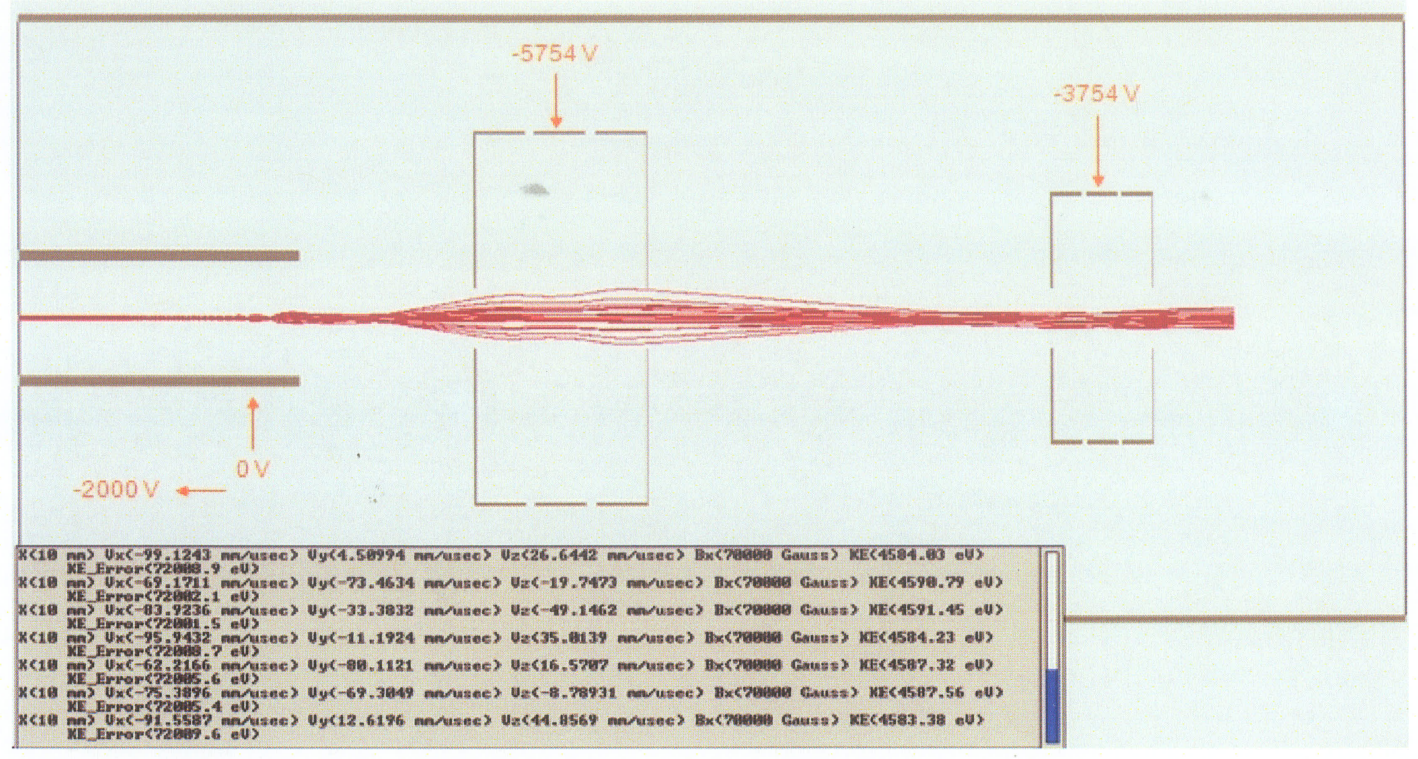

Figure 4.12: Krypton injection.

Simulation of electron injection

The injection system for electrons is the same as the proton injection system shown in

Figure 4.5. From Appendix A, the axial force only depends on the magnetic gradient and radial energy, if the energy is the same, because the electron is very light, its acceleration will be larger, and the electron velocity reduces quickly. Figure 4.13 shows that it is possible to reduce the single electron energy from $2.3 \mathrm{keV}$ to a few $\mathrm{eV}$. In this example, the voltage for the middle electrode of the first lens is $100 \mathrm{~V}$, the voltage for the middle electrode of the second lens is $3200 \mathrm{~V}$, the others are zero, and when the electron passes the center of drift tube, the voltage of the drift tube 
will be changed to 2000 V. Figure 4.14 shows that the electron energy can be reduced to a few hundred $\mathrm{eV}$ with $100 \%$ transportation efficiency when electrons are injected from a starting position $968 \mathrm{~mm}$ from the center of the magnetic field. The voltages of the lens system are all set to zero, the initial maximum radius of the electron beam is $4 \mathrm{~mm}$, the initial maximum angle of the electrons is $0.1^{\circ}$, and the initial electron energy is $2.3 \mathrm{keV}$. From Eq. 3.19 in section 3.1.5, electrons can cool themselves to ambient temperature by emitting synchrotron radiation. Figure 4.15 shows that 300 $\mathrm{eV}$ electrons can cool themselves to room temperature in about $0.6 \mathrm{~s}$.

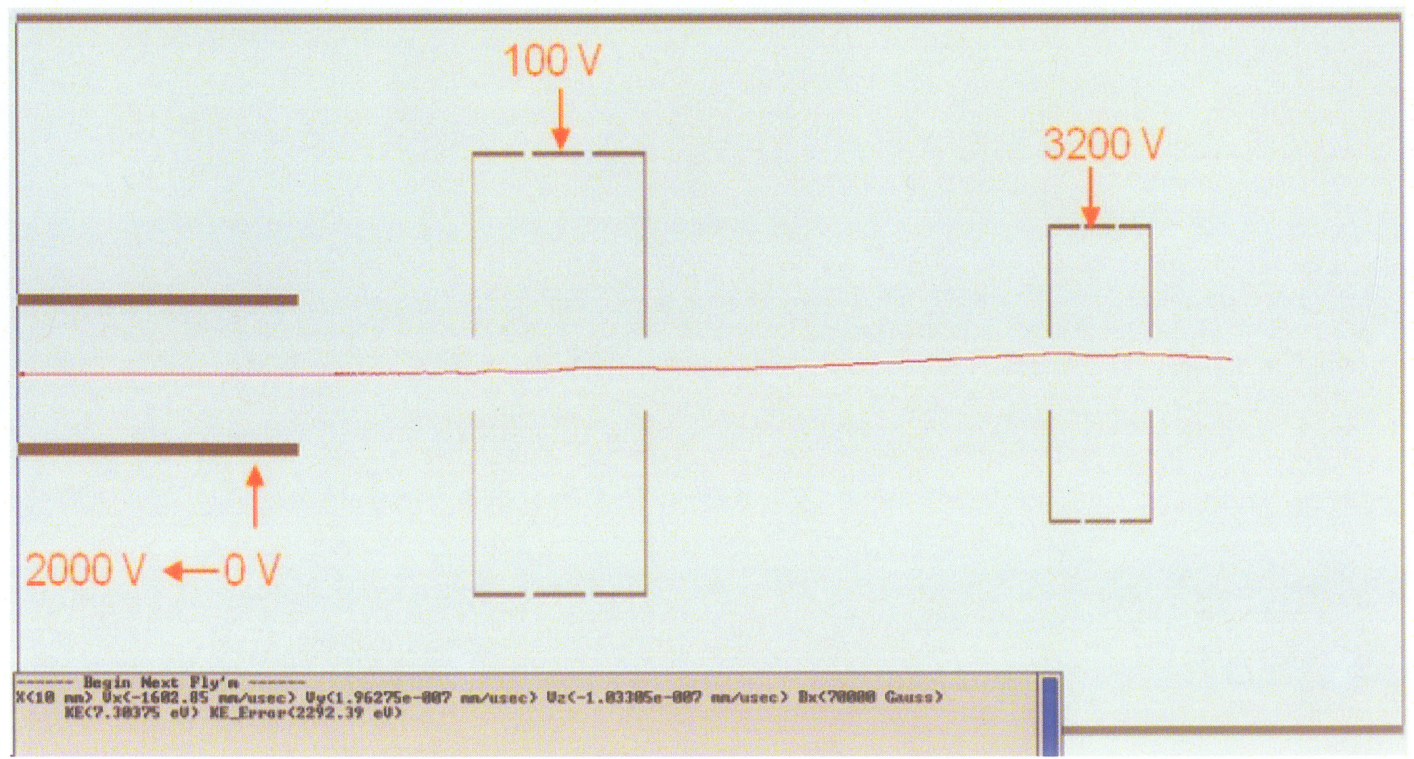

Figure 4.13: Single electron injection with an initial position $4 \mathrm{~mm}$ from the central line.

When injecting charged particles into the trap region, it is very difficult for very low-energy charged particles because of the large magnetic gradient region. For ions of 


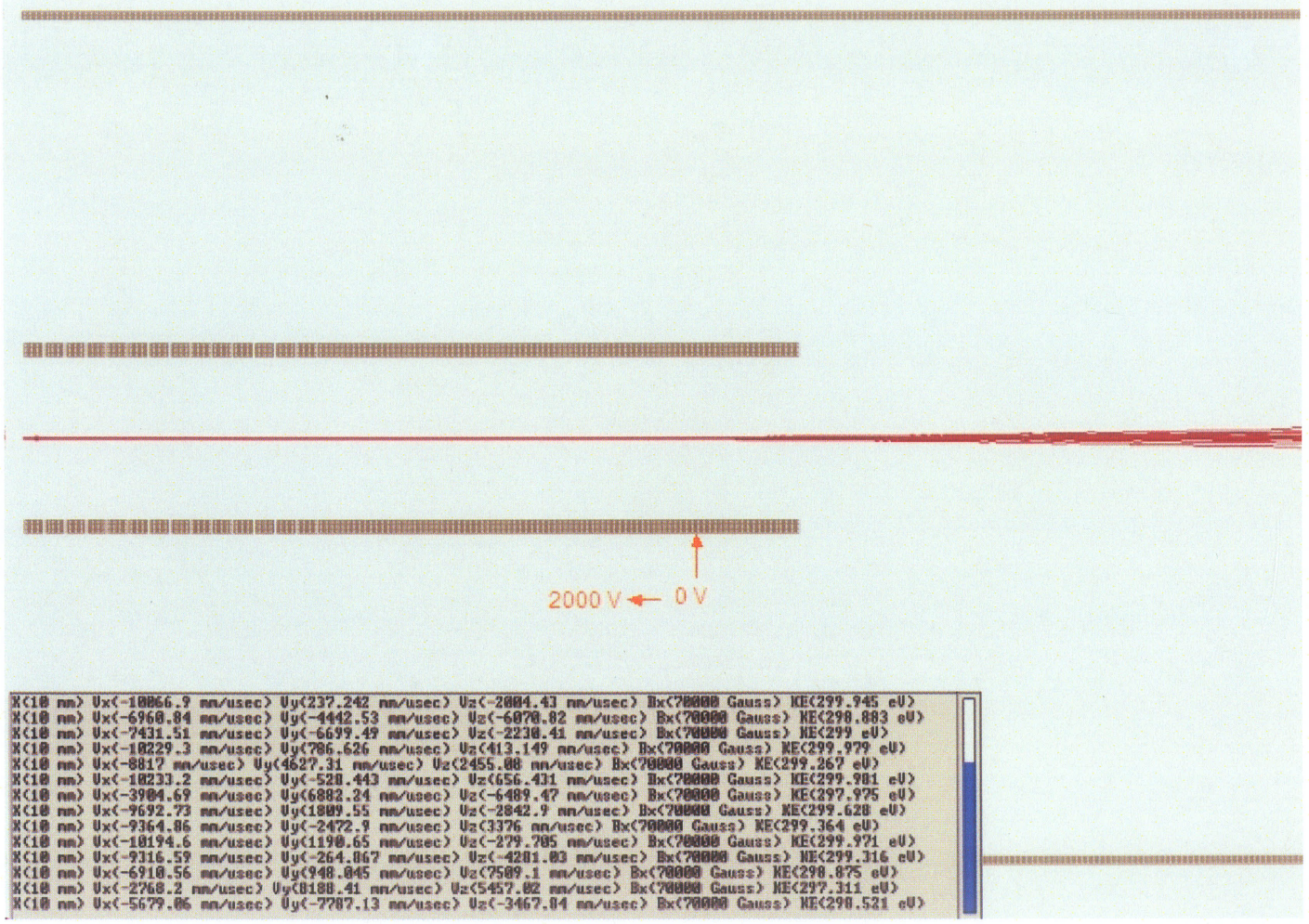

Figure 4.14: Electron injection. 


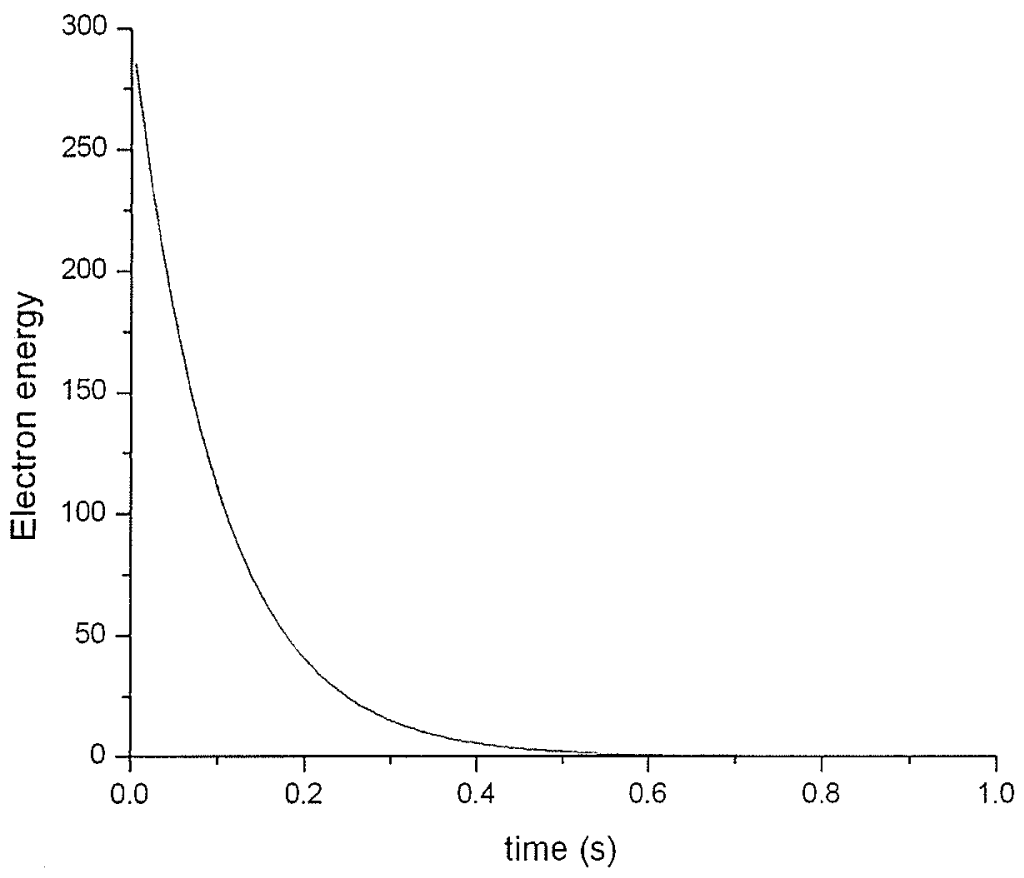

Figure 4.15: Electron synchrotron radiation. 
a few hundred eV energy this is possible for electron or proton cooling. For electrons, cooled by synchrotron radiation, one can also obtain cool electrons in the trap, but if we need low energy protons, only a certain percentage of the protons can be injected into the trap region with sufficiently low energy.

\subsection{Cooler trap implementation}

A layout of the cooler trap system under development is shown in Figure 4.16. It will allow electron cooling and proton cooling for highly charged ions, and it can be used in buffer gas cooling mode for singly charged ions. The trap consists of 28 cylindrical electrodes, each of them $19 \mathrm{~mm}$ in radius and $12.7 \mathrm{~mm}$ in length; the ratio of the radius to the length of the electrodes is set to about 1.42. A local harmonic potential can be generated anywhere in the cooler trap region (see section 4.2) as well as various nested well schemes. The trap works in transmission mode, i.e. HCI from the EBIT are injected from one side (left in Fig. 4.16) and after cooling they are ejected on the other side. This poses some difficulties, if three species of particles are to be injected separately, HCI, protons, and electrons. In the current design, $\mathrm{HCI}$ and protons enter from the same side, using a 4-way electrostatic beam switch. Electrons are inserted into the trap from the other side. The source needs to be off-axis to allow the HCI to exit. The electron gun is placed in the fringe field of the magnet, where it is possible to bring them on-axis with an electrostatic deflector.

At least initially, the cooler trap will be operated at room temperature like all 
TITAN components with the exception of the EBIT. HCI are very prone to charge change via electron capture from the rest gas, so special attention has to be paid to the vacuum conditions, in particular inside the trap, where the ions spend a significant amount of time. Vacuum on the order of $10^{-10}$ mbar or better is mandatory. Ion getter pumps with high pumping speed are located right outside the magnet and additional non-evaporable getter pumps (NEGs) are placed in the trap region inside the magnet.

The magnet for the cooler trap was delivered in March 2008. It was built by Cryomagnetics and has a large, homogeneous field region of 7 Tesla. Within a central volume of 4" length and 1" radius, the homogeneity is $10 \mathrm{ppm}$, and axially the field is homogeneous to $1000 \mathrm{ppm}$ in a $40 \mathrm{~cm}$ long region, permitting the use of a large trap structure necessary for implementing the nested wells and for the separate storage and preparation of different species, e.g. HCI and electrons. The magnetic-field sensitive environment at ISAC requires the use of an actively shielded magnet system. The 10 Gauss line of this magnet is on-axis 1.5 meters from the center and radially 1 meter. The magnet is embedded in a low-loss cryostat. The liquid helium and liquid nitrogen loss rates have been determined to be less than 1.5 litres per day and 5 litres per day, respectively. After charging the magnet in early May 2008, it has been running smoothly at 7 Tesla without problems. Figure 4.17 shows the superconducting magnet, the axial magnetic field is shown in Figure 4.6.

The design of all the vacuum parts and the electrode structure has been completed 
and is being submitted to the TRIUMF design shop in July 2008. Manufacture of the parts is anticipated by fall 2008 and assembly of the trap for spring 2009 .

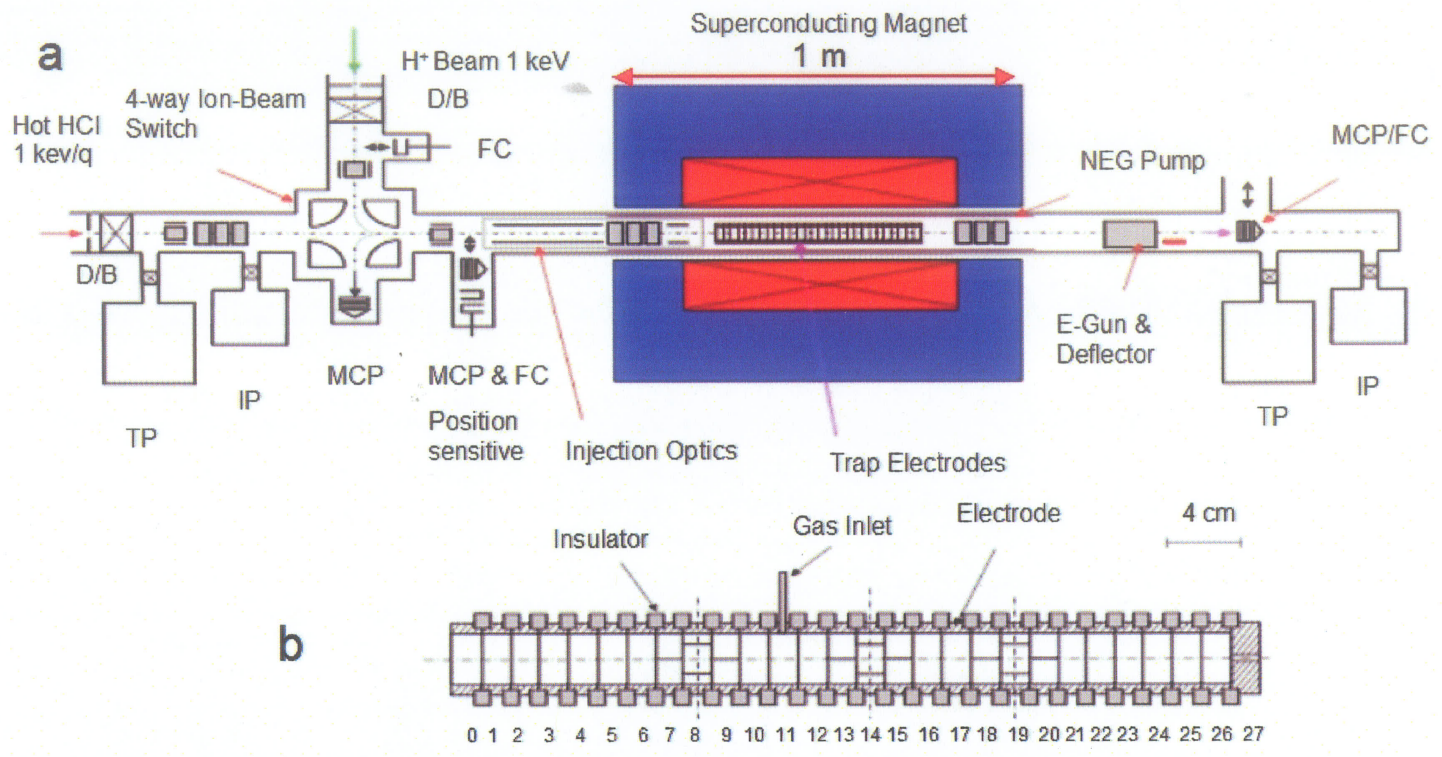

Figure 4.16: a) A schematic diagram for the cooler trap. FC, MCP, DB, TP, IP, EGun, NEG represent Faraday cup, multichannel plate, diffusion barrier, turbo pump, ion pump, field emission array, nonevaporative getter, respectively. b) The electrode structure. Electrodes 8, 14 and 19 are split into quadrants, and electrodes 7, 9, 13, 15,18 , and 20 into 2 segments. 


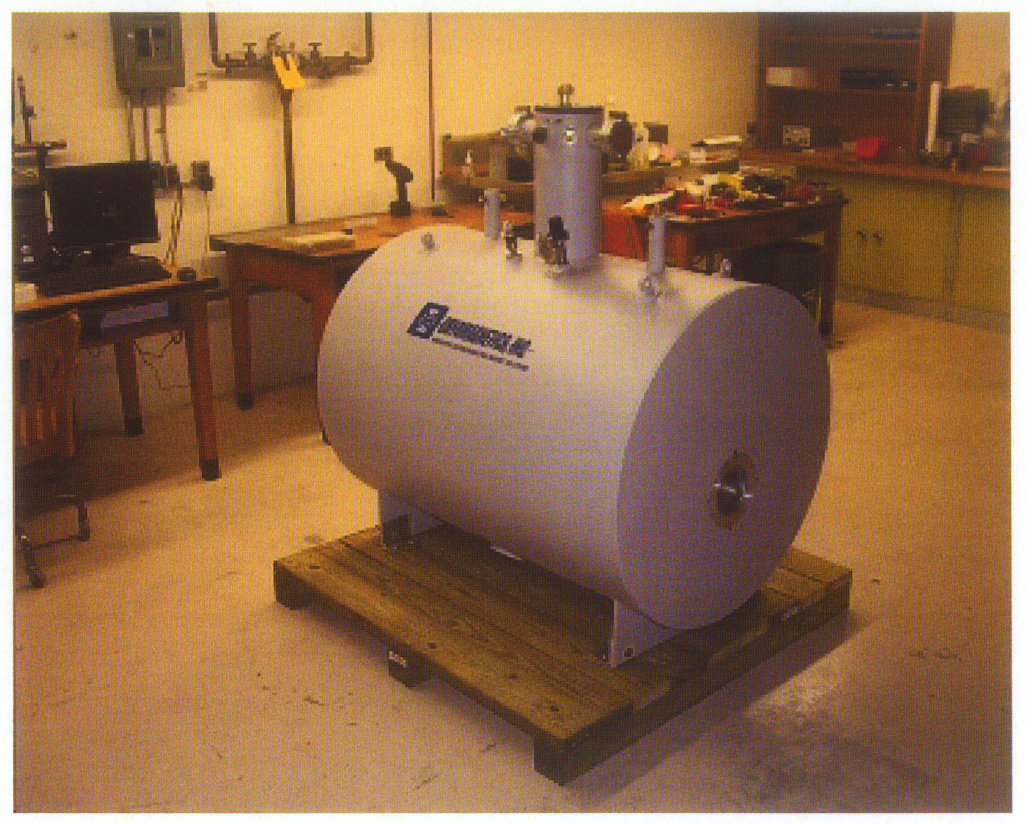

Figure 4.17: 7 Tesla superconducting magnet made by Cryomagnetics, Inc. at the University of Manitoba. The dewar has a length of $117 \mathrm{~cm}$ and a diameter of $86 \mathrm{~cm}$. 


\section{Chapter 5}

\section{MASS MEASUREMENT OF THE HALO NUCLEUS BERYLLIUM-11 AT TRIUMF}

Assembly of the cooler trap can only begin in 2009, and is beyond the time frame of this thesis. The startup of TITAN using singly charged ions provided the opportunity to work on some real mass data. A first focus of TITAN has been the light, radioactive halo nuclei ${ }^{8} \mathrm{He}^{+},{ }^{8} \mathrm{Li}^{+},{ }^{9} \mathrm{Li}^{+},{ }^{11} \mathrm{Li}^{+}$and ${ }^{11} \mathrm{Be}^{+}$, which have received a lot of attention in recent years, after the discovery of the first such nucleus, ${ }^{11} \mathrm{Li}$, by Tanihata in 1985 . In this chapter, we present a mass measurement evaluation for the ${ }^{11} \mathrm{Be}^{+}$ion, which provides a significant improvement in the determination of the mass excess.

The neutron or two-neutron separation energy for nuclei near the dripline may be less than $1 \mathrm{MeV}$ compared with the $6-8 \mathrm{MeV}$ observed in stable nuclei. The neutron density distribution in nuclei near the dripline has an extremely long tail, and the way this affects the reaction cross sections brings new properties to those nuclei. It is very interesting to study neutron halo candidates such as ${ }^{6} \mathrm{He},{ }^{11} \mathrm{Li},{ }^{11} \mathrm{Be},{ }^{14} \mathrm{Be},{ }^{17} \mathrm{~B}[111]$. ${ }^{11} \mathrm{Be}$ is a neutron halo nucleus [112] with a very small separation energy for the last neutron [113]. Its lifetime is $19.6 \mathrm{sec}$. It consists of an inert core and a loosely bound 
neutron. Table. 5.1 and Figure 5.1 show how the one neutron separation energy of Be changes with the mass number $A$. The only information on the halo of ${ }^{11} \mathrm{Be}$

Table 5.1: The neutron separation energy of beryllium isotopes.

\begin{tabular}{|l|l|}
\hline $\mathrm{A}$ & $S(n)(\mathrm{keV})$ \\
\hline 6 & 27690 \\
\hline 7 & 10676 \\
\hline 8 & 18899.68 \\
\hline 9 & 1665.3 \\
\hline 10 & 6812.29 \\
\hline 11 & 504 \\
\hline 12 & 3169 \\
\hline
\end{tabular}

comes from the measurement of the proton energy spectrum from the ${ }^{10} \mathrm{Be}(\mathrm{d}, \mathrm{p}){ }^{11} \mathrm{Be}$ reaction [28]. The mass excess of ${ }^{11} \mathrm{Be}$ was found to be $20.174 \pm 0.007 \mathrm{MeV}$, and this data was used for the AME2003 atomic mass evaluation $[113,114]$. A modern Penning trap experiment can do significantly better, and therefore ${ }^{11} \mathrm{Be}$ was chosen as one of the first cases for the new TITAN facility. 


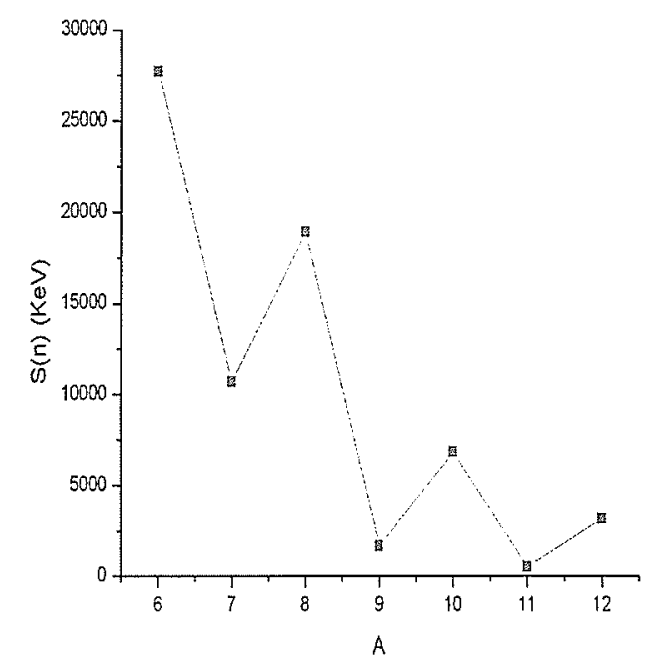

Figure 5.1: The one neutron separation energy of Be versus A.

\subsection{Measurement system of the TITAN Penning trap}

The bunched ions are brought to the Penning trap region by lens systems, then go through drift tubes and a Lorentz steerer, as shown in Figure 5.2, and into the Penning trap. From Eq. 2.57, if $R_{-}(0)$ is equal to zero, then the method of quadrupole excitation can not be used. One solution is to use dipolar excitation as soon as the ions enter the Penning trap and subsequently a quadrupole excitation. This, however, requires a certain dipolar excitation time, and for the mass measurement of short lifetime ions, the best way is to use the Lorentz steerer to be sure the ions reach the center of the Penning trap with finite $R_{-}(0)$. By applying a pulsed voltage to the drift tube near the Penning trap, the ions enter the Penning trap with a kinetic energy of 
$\sim 10 \mathrm{eV}$

After quadrupole excitation, the ions are extracted from the Penning trap by applying a voltage pulse at the end-cap electrodes. The pulse is used as the starting time of the ion TOF. The extraction system is shown in Figure 5.3. Ions go through the drift tube and lens and are recorded by the MCP. The signal recorded by the MCP is used as the stopping time of the ion TOF. Figure 5.4 shows the structure of the TITAN Penning trap. The ideal azimuthal quadrupole potential described in section 2.3.2 is created by the electrodes of the Penning trap together with some correction electrodes.

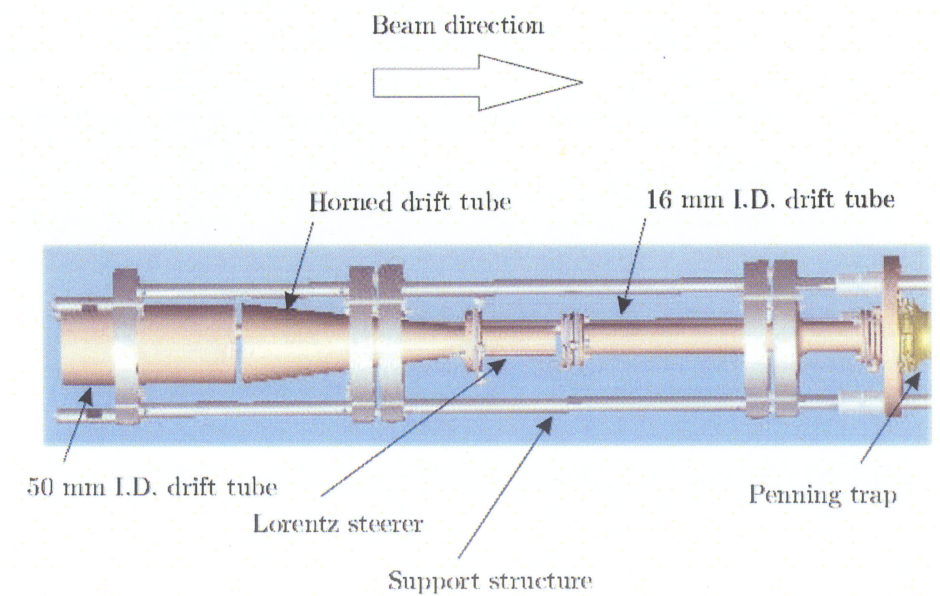

Figure 5.2: Injection system of the TITAN Penning trap. Courtesy of M. Brodeur, TITAN collaboration. 

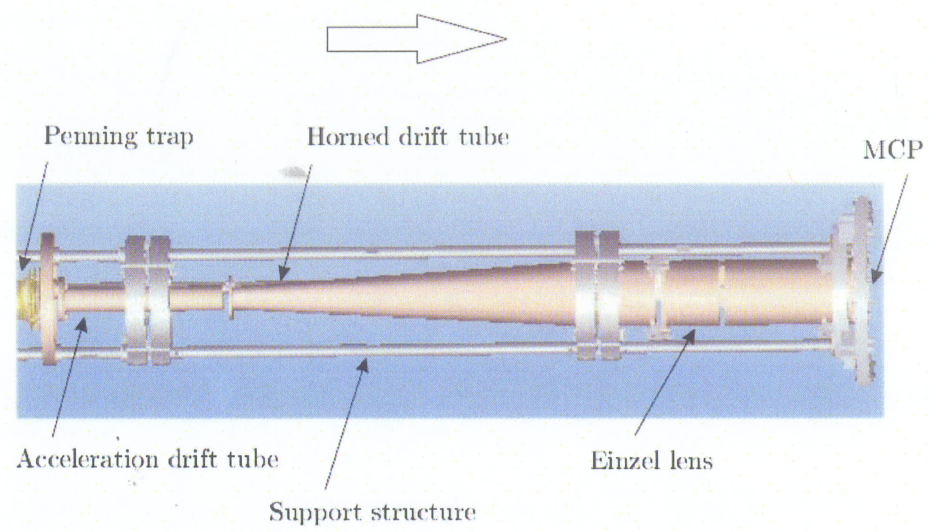

Figure 5.3: Extracting system of the TITAN Penning trap. Courtesy of M. Brodeur, TITAN collaboration.

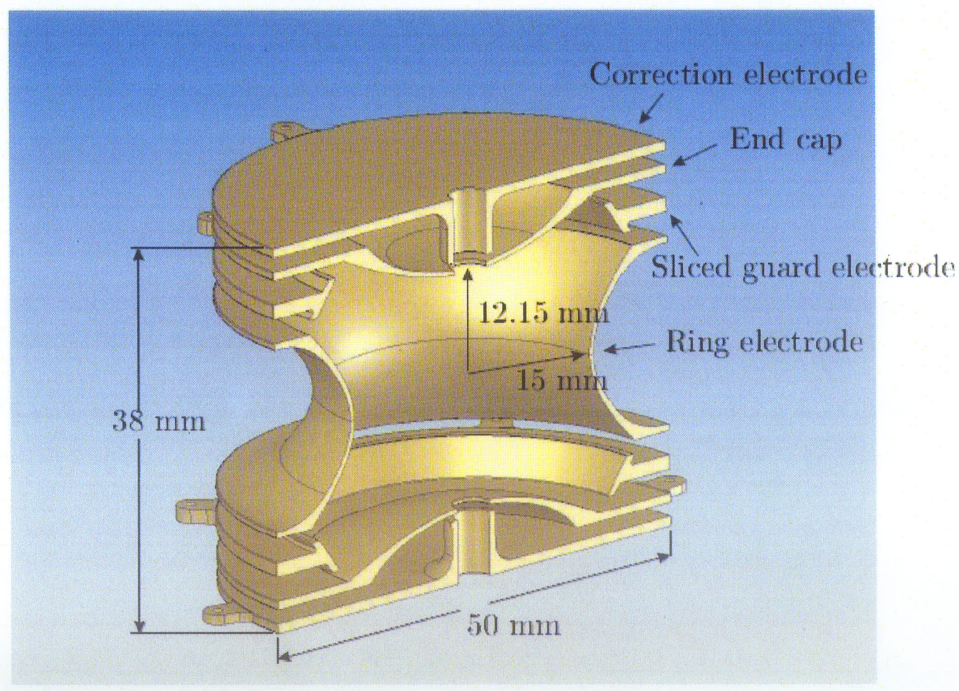

Figure 5.4: Structure of the Penning trap used at TITAN. Courtesy of M. Brodeur, TITAN collaboration. 


\subsection{Analysis method}

From the TOF spectrum of the ion to be measured and the TOF spectrum of the reference ion, we can obtain the cyclotron frequencies of the measured and reference ions by fitting to a known function. By comparing with the mass of the known reference ion, and taking into account systematic errors and statistical errors, the ion mass can be obtained as shown below. The statistical Birge ratio can help to indicate whether the uncertainty is overestimated or underestimated.

The error analysis for the early TITAN measurements follows the procedures developed for ISOLTRAP $[115,41]$. For the individual measurement, $\sigma\left(\gamma_{r e f}^{i}\right)$ and $\sigma\left(\gamma^{i}\right)$ are respectively the statistical uncertainties of the reference ion measurement and the ion measurement and include uncertainties due to the number of the ions in the Penning trap. The correction for ion number is made using Z-class analysis, which uses data showing the relation between the cyclotron frequency and the number of ions per shot and creates a function by fitting this data (the number of ions per shot can be obtained from the multiplicity of the MCP event). The fitted function is then used to extrapolate to one ion, and the cyclotron frequency and its statistical uncertainty for one ion in the Penning trap are determined. However, for the ${ }^{11} \mathrm{Be}$ runs, the ion number was so low that Z-class analysis was not performed. $\sigma_{B}\left(\gamma_{r e f}^{i}\right)$ is the standard uncertainty caused by the magnetic field fluctuation. $\sigma_{m}(\bar{r})$ is the uncertainty which depends on the mass difference between the ion measured and the 
reference ion. $\sigma_{\text {res }}(\bar{r})$ is a residual systematic effect.

The ratio of the cyclotron frequency of the ion measured to that of the reference ion is given by

$$
r^{i}=\frac{\gamma_{r e f}^{i}}{\gamma_{i}}
$$

The error $\sigma_{c}\left(\gamma_{r e f}^{i}\right)$, which is related to the reference ion individual measurement, is given by

$$
\frac{\sigma_{c}\left(\gamma_{r e f}^{i}\right)}{\gamma_{r e f}^{i}}=\sqrt{\left[\frac{\sigma\left(\gamma_{r e f}^{i}\right)}{\gamma_{r e f}^{i}}\right]^{2}+\left[\frac{\sigma_{B}\left(\gamma_{r e f}^{i}\right)}{\gamma_{r e f}^{i}}\right]^{2}},
$$

then the error $\sigma_{c}\left(r^{i}\right)$ of the individual ion measurement is given by

$$
\frac{\sigma_{c}\left(r^{i}\right)}{r^{i}}=\sqrt{\left[\frac{\sigma\left(\gamma^{i}\right)}{\gamma^{i}}\right]^{2}+\left[\frac{\sigma_{c}\left(\gamma_{r e f}^{i}\right)}{\gamma_{r e f}^{i}}\right]^{2}} .
$$

The average ratio of the cyclotron frequency of the ion measured to that of the reference ion is given by

$$
\bar{r}=\frac{\sum_{i} \frac{r^{i}}{\sigma_{c}^{2}\left(r^{i}\right)}}{\sum_{i} \frac{1}{\sigma_{c}^{2}\left(r^{i}\right)}} .
$$

The average error $\sigma(\bar{r})$ can be given by

$$
\frac{\sigma(\bar{r})}{\bar{r}}=\frac{1}{\bar{r}} \frac{1}{\sqrt{\sum_{i \frac{1}{\sigma_{c}^{2}\left(r^{i}\right)}}}} .
$$

Finally, the final error $\sigma_{c}(\bar{r})$, which includes the standard uncertainty caused by the magnetic field fluctuations and residual systematic effects, can be given by

$$
\frac{\sigma_{c}(\bar{r})}{\bar{r}}=\sqrt{\left[\frac{\sigma(\bar{r})}{\bar{r}}\right]^{2}+\left[\frac{\sigma_{m}(\bar{r})}{\bar{r}}\right]^{2}+\left[\frac{\sigma_{r e s}(\bar{r})}{\bar{r}}\right]^{2}} .
$$

The ion mass is given by

$$
M_{i o n}=\frac{q_{i o n}}{q_{r e f}} \cdot \bar{r} \cdot\left(M_{r e f}-q_{r e f} \cdot m_{e}+B_{e}(r e f) / c^{2}\right)+q_{i o n} \cdot m_{e}-B_{e}(i o n) / c^{2},
$$


where $q_{i o n}$ is the charge number of the ion, $q_{r e f}$ is the charge number of the reference ion, $M_{r e f}$ is the mass of the reference ion, $m_{e}$ is the electron mass, $B_{e}$ (ref) is the total electron binding energy of the reference ion, and $B_{e}$ (ion) is the total electron binding energy of the ion.

The mass uncertainty is given by

$$
\Delta M_{i o n}=\frac{q_{i o n}}{q_{r e f}} \sqrt{\left(M_{r e f} \cdot \sigma_{c}(\bar{r})\right)^{2}+\left(\bar{r} \cdot \Delta_{M_{r e f}}\right)^{2}}
$$

where $\Delta_{M_{\text {ref }}}$ is the mass uncertainty of the reference ion.

The Birge ratio is given by

$$
R=\frac{\sigma_{o u t}}{\sigma_{\text {in }}}
$$

where $\sigma_{\text {out }}$ is given by

$$
\sigma_{\text {out }}=\sqrt{\frac{\sum_{i} \frac{\left(r^{i}-\tilde{r}\right)^{2}}{\sigma_{c}^{2}\left(r^{i}\right)}}{(N-1) \sum_{i} \frac{1}{\sigma_{c}^{2}\left(r^{i}\right)}}}
$$

and $\sigma_{i n}$ is given by

$$
\sigma_{i n}=\sqrt{\frac{1}{\sum_{i} \frac{1}{\sigma_{c}^{2}\left(r^{2}\right)}}} .
$$

When the Birge ratio is more than one, it means there is some uncertainty which not been included; when the Birge ratio is less than one, it means the uncertainty is overestimated.

\section{3 $\quad{ }^{11} B e$ data analysis}

After the mass measurements, the cyclotron frequencies of the measured ion and the reference ion are extracted from the TOF spectra. We use a program, EVA, to fit the 
TOF spectrum which uses the MIKE function shown in Eq. 2.60 to calculate the time of flight, taking into account the interaction between the ion and the background gas. The radial energy, $E_{r}$, can be derived in the same way as used to obtain Eq. 2.55 by adding the average damping force [36]

$$
\vec{F}=-\delta \cdot m \cdot \dot{\vec{r}}
$$

where $\delta$ is the damping coefficient.

The MIKE function depends on $V(z)$, which we can get for a given Penning trap extraction system by using SIMION. Figure 5.5 shows the axial potential $V(z)$ for the system setup described in Figure 2.13, and is based on the real TITAN setup. $B(z)$ is known, and from Eq.2.55, $E_{0}$ is related to the initial $R_{+}$and $R_{-}$, so after some variables are known, the time of flight can be calculated using the MIKE function. When using MIKE function fitting, we leave some parameters variable and some fixed. The EVA control is shown in Figure 5.6. The MCA range decides the TOF range we can record, so only TOF data which fall in this range will be taken into account. Figure 5.7 shows the TOF spectrum for run 033066, which is a mass measurement of ${ }^{6} \mathrm{Li}^{+}$. The blue line in Figure 5.7 shows the TOF range selected by the MCA range in EVA; the total number of ${ }^{6} \mathrm{Li}^{+}$ions falling in this range is 2116 . Figure 5.8 shows the TOF spectrum for run 033072 , which is a mass measurement of ${ }^{11} \mathrm{Be}^{+}$. Here, the total number of ${ }^{11} \mathrm{Be}^{+}$ions falling in the selected range is 619 . The detector only records a few ions at a time. Figure 5.9 shows the number of ${ }^{6} \mathrm{Li}^{+}$ions per shot for run 033066 ; the maximum is about 8 . Figure 5.10 shows the number of ${ }^{11} \mathrm{Be}^{+}$ions per shot for run 
033072; the maximum is about 3 . A full scan consists of measurements of 41 frequency points, and the TOF spectrum consists of many scans. FitPar in Figure 5.6 shows the parameters for the MIKE function fit, the checked ones are variable, the others are fixed. Center, Rho-, Rho+, Conv, ADamp, TRF, and Tofoff are respectively the central frequency $(\mathrm{Hz})$ of the MIKE function, the initial radius (mm) of the magetron motion, initial radius ( $\mathrm{mm}$ ) of the reduced cyclotron motion, the conversion number from magnetron motion to reduced cyclotron motion, the damping coefficient $(\mathrm{Hz})$, the time length $(\mathrm{s})$ of the quadrupole excitation and the base-line time of flight $(\mu s)$. The duration of the quadrupole excitation for ${ }^{11} \mathrm{Be}^{+}$is $0.498 \mathrm{~s}$.

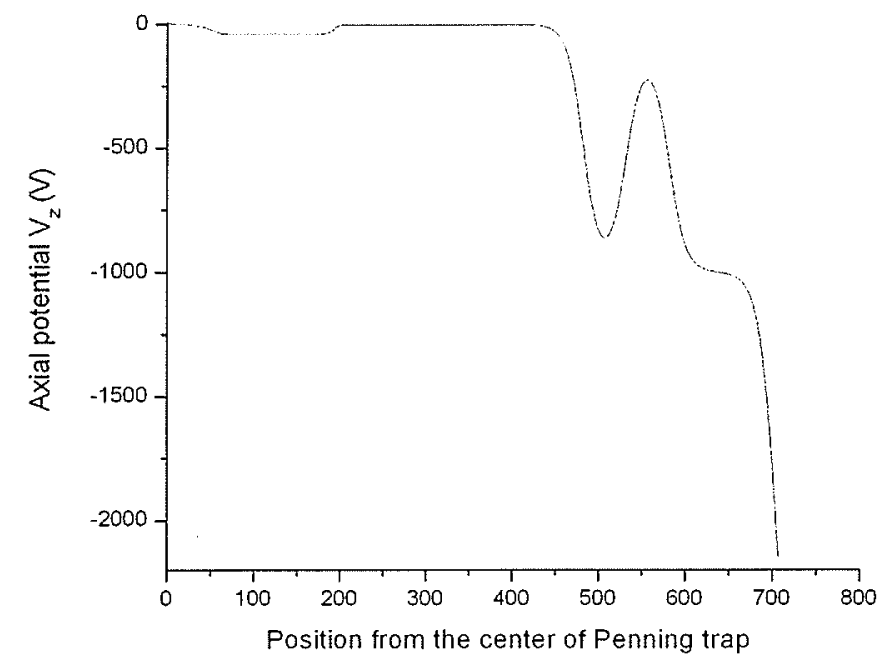

Figure 5.5: Penning trap axial potential.

Figure 5.11(a) shows the TOF measurement for the reference ion ${ }^{6} \mathrm{Li}^{+}$. After MIKE function fitting, a file, run033066_eva.ft2, will be created. The file includes 


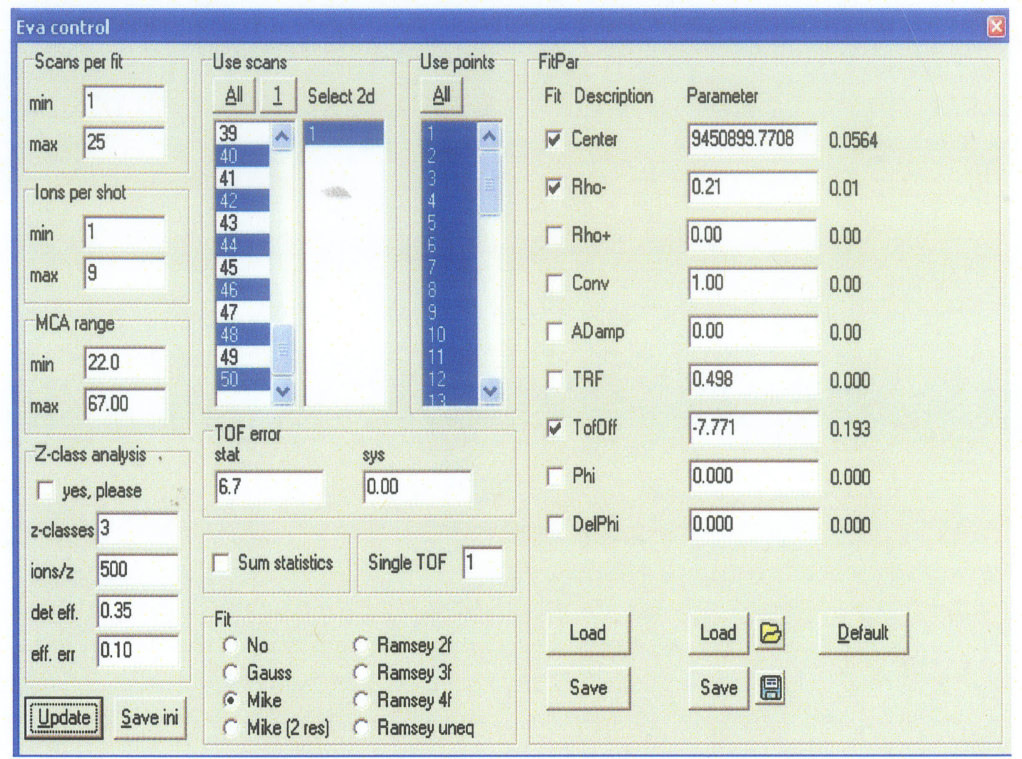

Figure 5.6: EVA control.

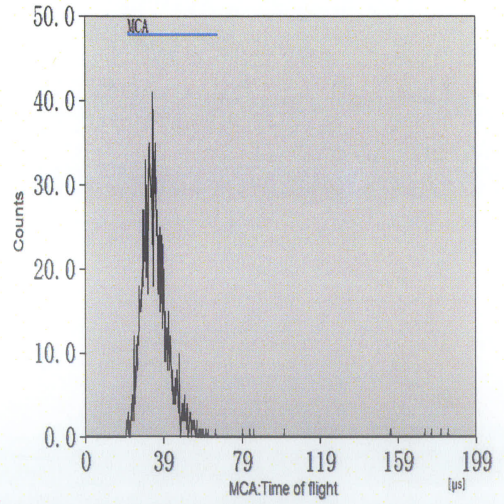

Figure 5.7: Counts vs TOF for run 033066.

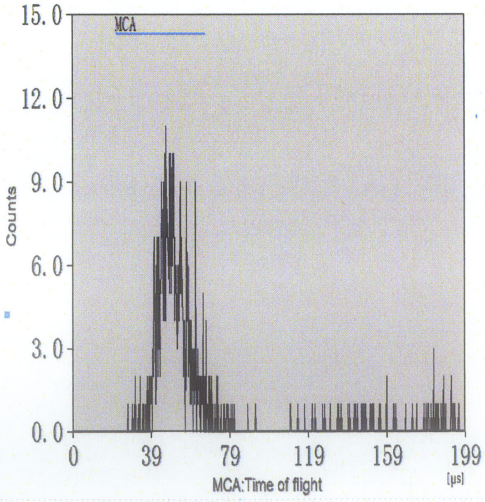

Figure 5.8: Counts vs TOF for run 033072. 


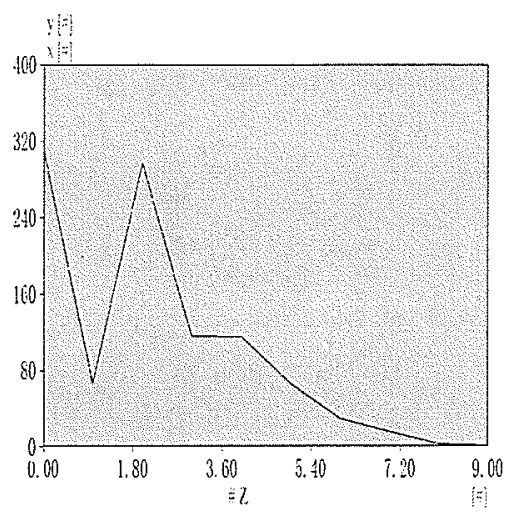

Figure 5.9: The spectrum of ion number per shot for run 033066 .

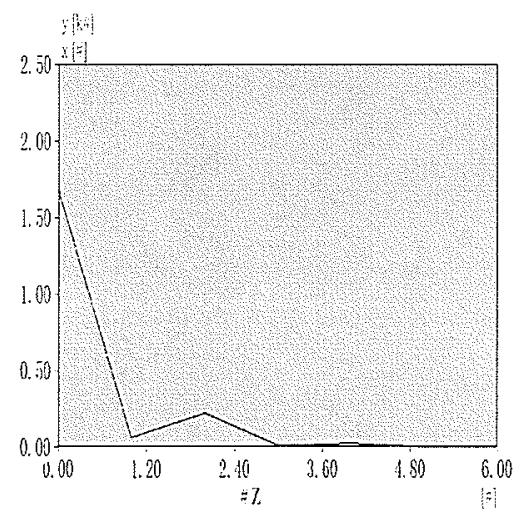

Figure 5.10: The spectrum of ion number per shot for run 033072 .

the center frequency, error of the center frequency, and measurement time information, which are needed for the ion mass calculation. Figure 5.11(b) shows another TOF measurement for the reference ion ${ }^{6} \mathrm{Li}^{+}$. Figure 5.12 (a), Figure 5.12 (b), and Figure 5.12 (c) show TOF measurements for the ion ${ }^{11} \mathrm{Be}^{+}$. These measurements were made in a specific order: the TOF measurements of the ion ${ }^{11} \mathrm{Be}^{+}$ were made between the two TOF measurements of the reference ion ${ }^{6} \mathrm{Li}^{+}$. In the same way as run033066_eva.ft2, after fitting, run033074_eva.ft2, run033069_eva.ft2, run033070_eva.ft2 and run033072_eva.ft2 were created.

After the .ft2 files are created by EVA, cyclotron frequencies $\gamma_{\text {ref }}^{i}$ and $\gamma^{i}$, and their errors $\sigma\left(\gamma_{r e f}^{i}\right)$ and $\sigma\left(\gamma^{i}\right)$ are all included in the files. The program SOMA (Simple Online Mass Analysis) loads these files and calculates the ion mass and the uncertainty according to Eq. 5.7 and Eq. 5.8 .

The SOMA window is shown in Figure 5.13. Two reference measurement files of 

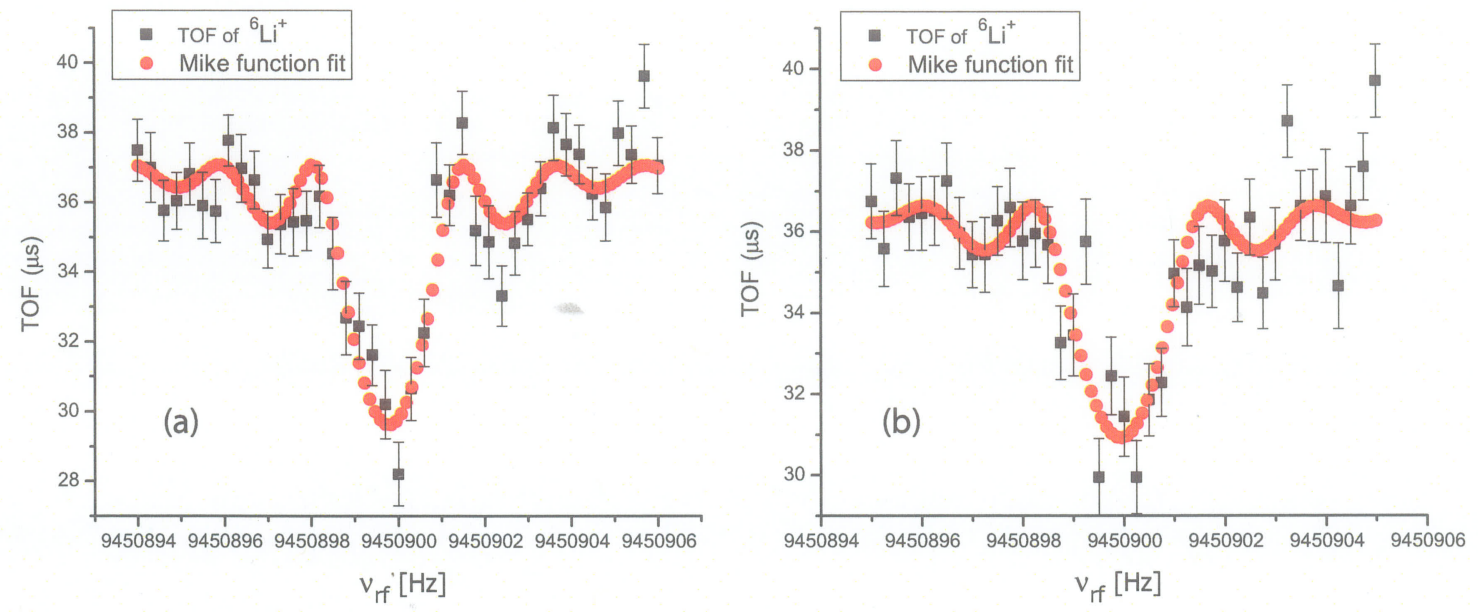

Figure 5.11: (a) TOF of reference ion ${ }^{6} \mathrm{Li}^{+}$for run 033066. (b) TOF of reference ion ${ }^{6} \mathrm{Li}^{+}$for run 033074 .

${ }^{6} \mathrm{Li}^{+}$as a calibration and three ion TOF measurement files of ${ }^{11} \mathrm{Be}^{+}$are entered for our analysis. The electron binding energies for $\mathrm{Li}$ and Be were taken as $5.391719 \mathrm{eV}$ and $9.3227 \mathrm{eV}$, respectively. Systematic effects have to be considered: The fractional change of the magnetic field with time which was determined to be $-2 \times 10^{-9}$ per hour for TITAN magnet and additional miscellaneous systematic errors, estimated to be about $5 \times 10^{-8}$ for TITAN at this stage [116]. More details about SOMA are described in reference [41].

With this analysis, the mass excess of ${ }^{11} \mathrm{Be}$ is determined to be $20176.936(0.531)$ $\mathrm{keV}$ compared to the old value $20174(7) \mathrm{keV}$ [28], and is shown in Figure 5.14. The Birge ratio of 2.364 indicates that further studies of systematic errors are needed. Nevertheless, this already constitutes an improvement of about one order of magnitude over the AME03 value. 

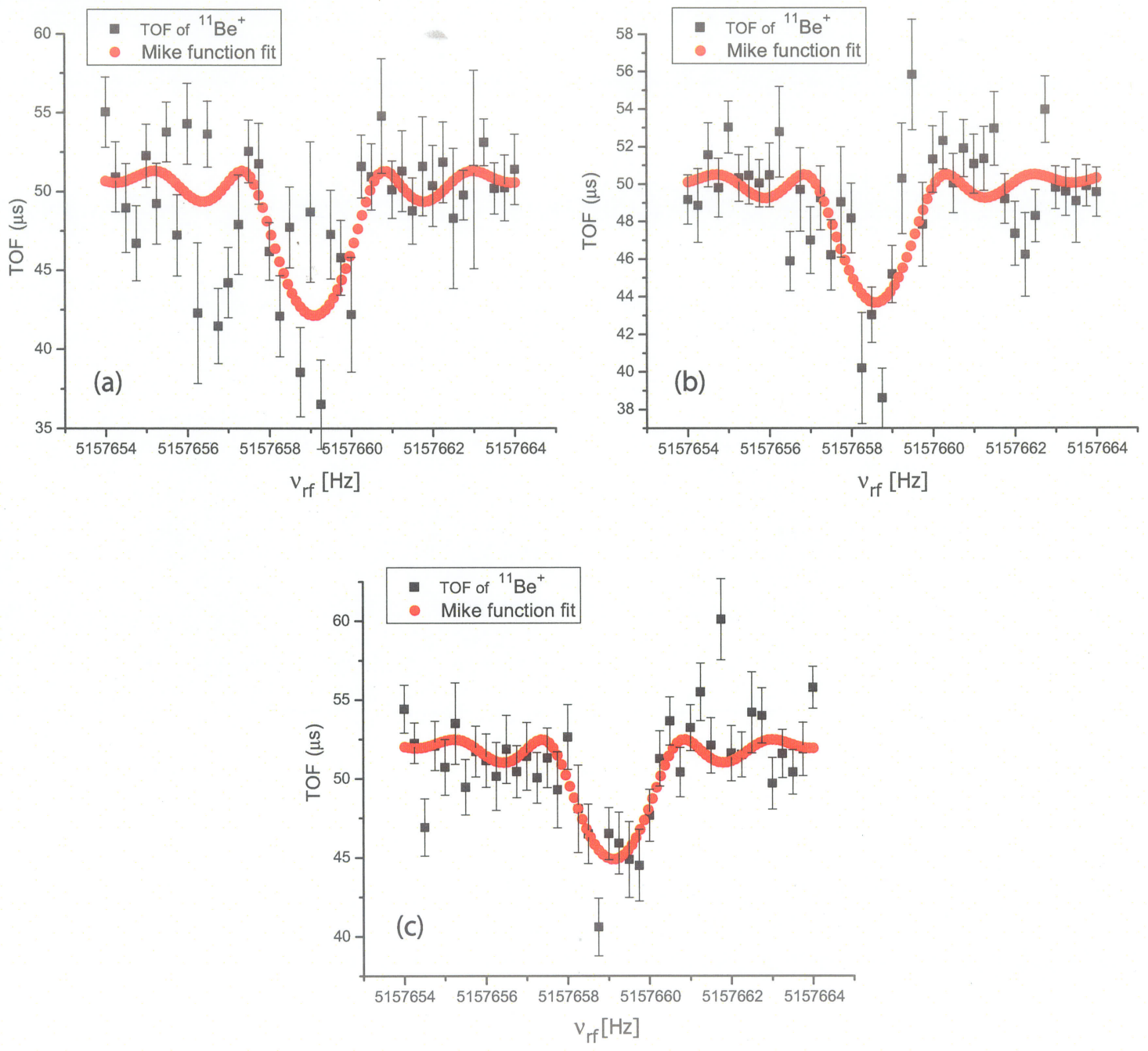

Figure 5.12: (a) TOF of ion ${ }^{11} \mathrm{Be}^{+}$for run 033069. (b) TOF of ion ${ }^{11} \mathrm{Be}^{+}$for run 033070. (c) TOF of ion ${ }^{11} \mathrm{Be}^{+}$for run 033072. 


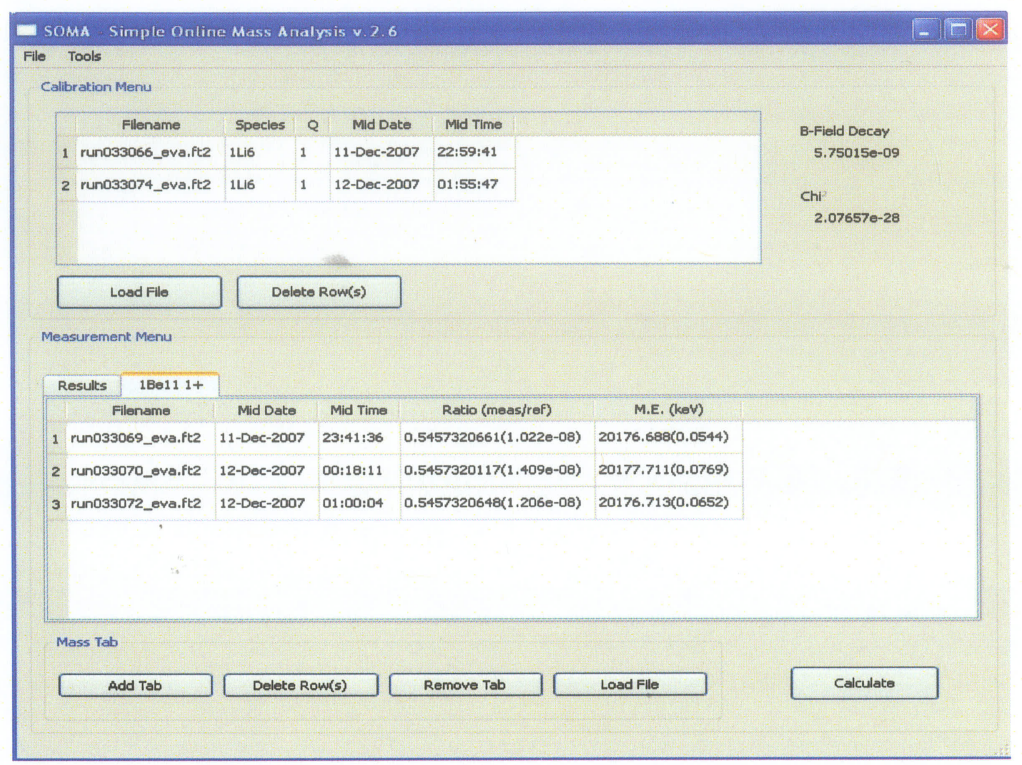

Figure 5.13: SOMA window for loading files.

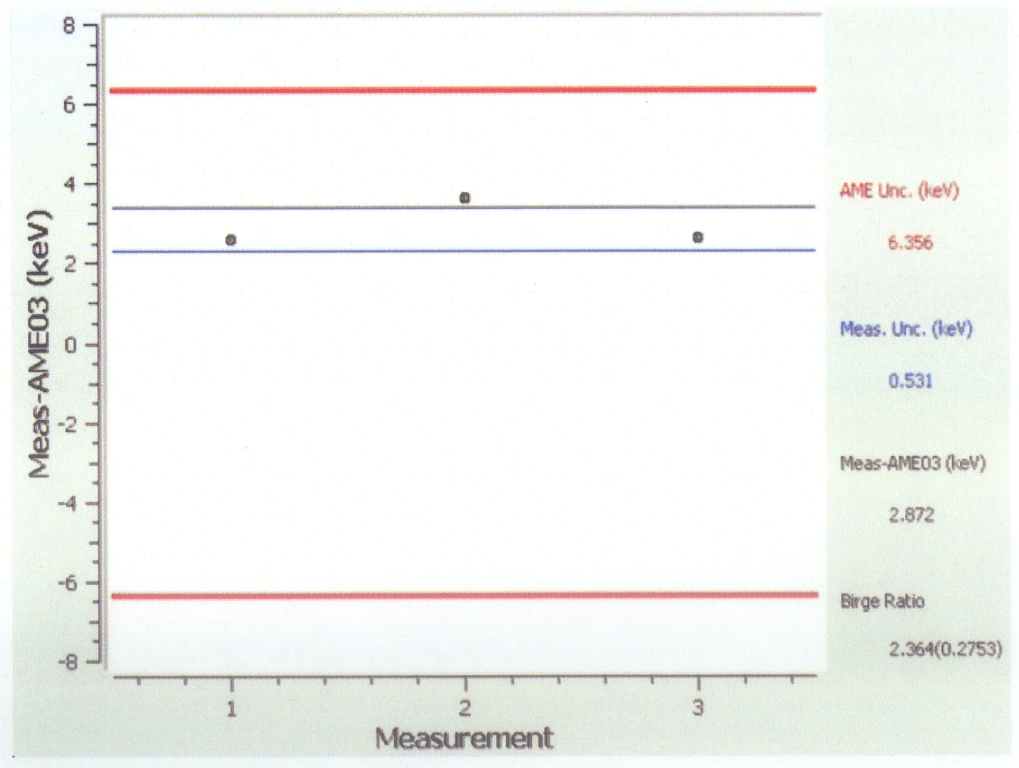

Figure 5.14: SOMA window for results. 


\section{Chapter 6}

\section{SUMMARY AND OUTLOOK}

We have designed a cooler ion trap for the TITAN project. Electron and proton cooling were investigated in detail. For proton cooling under the conditions of proton density $=10^{8} \mathrm{~cm}^{-3}$, proton number $=10^{8}$, initial proton temperature $=1 \mathrm{eV}$, number of $\mathrm{HCI}=10^{3}$, and initial energy of $\mathrm{HCI}=500 \mathrm{eV} / q$, in proton cooling of ions such as $\mathrm{C}^{6+}, \mathrm{Fe}^{23+}, \mathrm{Kr}^{36+}$ and $\mathrm{U}^{89+}$, the cooling time is about $0.15 \mathrm{~s}-2.3 \mathrm{~s}$ depending on the ion. For electron cooling under the conditions of electron density $=10^{7} \mathrm{~cm}^{-3}$, electron number $=10^{7}$, initial electron temperature $=300 \mathrm{~K}$ (ambient temperature) number of $\mathrm{HCI}=10^{3}$, initial energy of $\mathrm{HCI}=500 \mathrm{eV} / q$, in electron cooling of ions

such as $\mathrm{C}^{6+}, \mathrm{Fe}^{23+}, \mathrm{Kr}^{36+}$ and $\mathrm{U}^{89+}$, the cooling time is about $0.24 \mathrm{~s}-0.35$ s depending on the ion. Compared to electron cooling of ions, more protons are needed to cool highly charged ions to the same energy. This technique has not been demonstrated yet, and it remains to be seen whether sufficiently dense samples of cold protons can be trapped. The effects of electron-ion recombination in electron cooling have been modeled and we find that there is a time window during which effective cooling takes place but little recombination. The initially hot HCI first heat up the electrons, strongly suppressing recombination, which only sets in once the HCI have cooled 
down to well below $1 \mathrm{eV} / q$. This creates an opportunity to separate the species before charge-state changes set in. Electron cooling has been established for antihydrogen production and the prospects for extending it to highly charged ions look promising.

A method has been developed to design arbitrary harmonic or nested well potentials with a 28-electrode, cylindrical Penning trap. This will allow great flexibility to investigate different scenarios with protons and electrons.

Efficient transport of charged particles through a strong magnetic field gradient without pick-up of radial energy requires a careful design of the injection and ejection sections. Extensive simulations of the injection of highly charged ions of $\mathrm{keV} / q$ energy into a trap with high magnetic field, show that injection of highly charged ions with a final energy of a few hundred $\mathrm{eV} / \mathrm{q}$ can readily be achieved with $100 \%$ efficiency. They are suitable for further electron cooling or proton cooling. Highly-efficient injection of electrons is also achieved as they can have sufficiently high energy (few hundred $\mathrm{eV})$. Once they are in the trap, they can self-cool via synchrotron radiation. Most problematic is the injection of protons, as they must not have more than $10-20 \mathrm{eV}$ of kinetic energy once they arrive in the trap, as they cannot radiate away energy. We have found a geometry where $80 \%$ of the protons with emittance of about $10 \pi \mathrm{mm}$ mrad can be injected into the trap region, with a final proton energy of about $16 \mathrm{eV}$.

Based on these investigations, a cooler trap was designed and is ready for submission to the TRIUMF design shop. Assembly is anticipated early in 2009. The 7 Tesla 
magnet has been delivered and runs smoothly.

TITAN has started taking data with singly-charged ions. We have measured the mass of the short-lived halo nucleus ${ }^{11} \mathrm{Be}$. Its mass excess is determined to be $20176.936(0.531) \mathrm{keV}$, an order of magnitude more accurate than the AME03 value. An ion source providing test beams for the TITAN EBIT and the cooler trap has been constructed (see Appendix F). We characterized the source and found a favorable emittance of less than $10 \pi \mathrm{mm}$ mrad. 


\section{Chapter 7}

\section{APPENDIX}

\subsection{Appendix A: Axial force depending on a magnetic gradient}

When an ion moves in a magnetic field [117], the motion perpendicular to the magnetic field can be given by

$$
m \frac{v_{\perp}^{2}}{\rho}=q v_{\perp} B
$$

where $B$ is the axial magnetic field, $v_{\perp}$ is the velocity component perpendicular to the magnetic field, and $\rho$ is the radius of curvature. From Eq. 7.1, the radius of curvature is given by

$$
\rho=\frac{m v_{\perp}}{q B}
$$

The circular motion frequency $\omega_{c}$ is given by

$$
\omega_{c}=\frac{v_{\perp}}{\rho}
$$

The ion's magnetic moment, which is equal to the current times the area, is given by

$$
\mu=I A
$$

where

$$
I=\frac{q}{T}=\frac{q^{2} B}{2 \pi m}
$$


and

$$
A=\pi \rho^{2}=\pi\left(\frac{m v_{\perp}}{q B}\right)^{2} .
$$

Then we can get

$$
\mu=\frac{m v_{\perp}^{2}}{2 B}=\frac{E_{r}}{B} .
$$

From Maxwell's equation

$$
\nabla \cdot B=0
$$

we have

$$
\nabla \cdot B=\frac{1}{\rho} \frac{\partial\left(\rho B_{\rho}\right)}{\partial \rho}+\frac{\partial B_{z}}{\partial z}=0 .
$$

From Eq. 7.9, then we can get

$$
B_{\rho}=-\frac{1}{2}\left(\frac{\partial B_{z}}{\partial z}\right) \rho
$$

from Eq. 7.10, we have

$$
B_{x}=-\frac{1}{2}\left(\frac{\partial B_{z}}{\partial z}\right) x
$$

and

$$
B_{y}=-\frac{1}{2}\left(\frac{\partial B_{z}}{\partial z}\right) y
$$

Parallel motion is given by

$$
m \frac{d v_{z}}{d t}=F_{z}=q\left[v_{x} B_{y}-v_{y} B_{x}\right] .
$$

So we have

$$
F_{z}=q\left[v_{x} B_{y}-v_{y} B_{x}\right]
$$


where

$$
\begin{gathered}
x=\rho \sin \omega_{c} t, \\
y=\rho \cos \omega_{c} t, \\
v_{x}=\omega_{c} \rho \cos \omega_{c} t, \\
v_{y}=-\omega_{c} \rho \sin \omega_{c} t,
\end{gathered}
$$

from above, we can get

$$
F_{z}=-\frac{\partial B_{z}}{\partial z}\left(\frac{q}{2} \omega_{c} \rho^{2}\right)
$$

From Eq. 7.2, Eq. 7.3, and Eq. 7.7, the final expression for the axial force is

$$
F_{z}=-\mu \frac{\partial B_{z}}{\partial z}=-\frac{E_{r}}{B} \frac{\partial B_{z}}{\partial z}
$$

where $E_{r}=\frac{1}{2} m v_{\perp}^{2}$.

\subsection{Appendix B: Geometry file for simulation of time of flight}

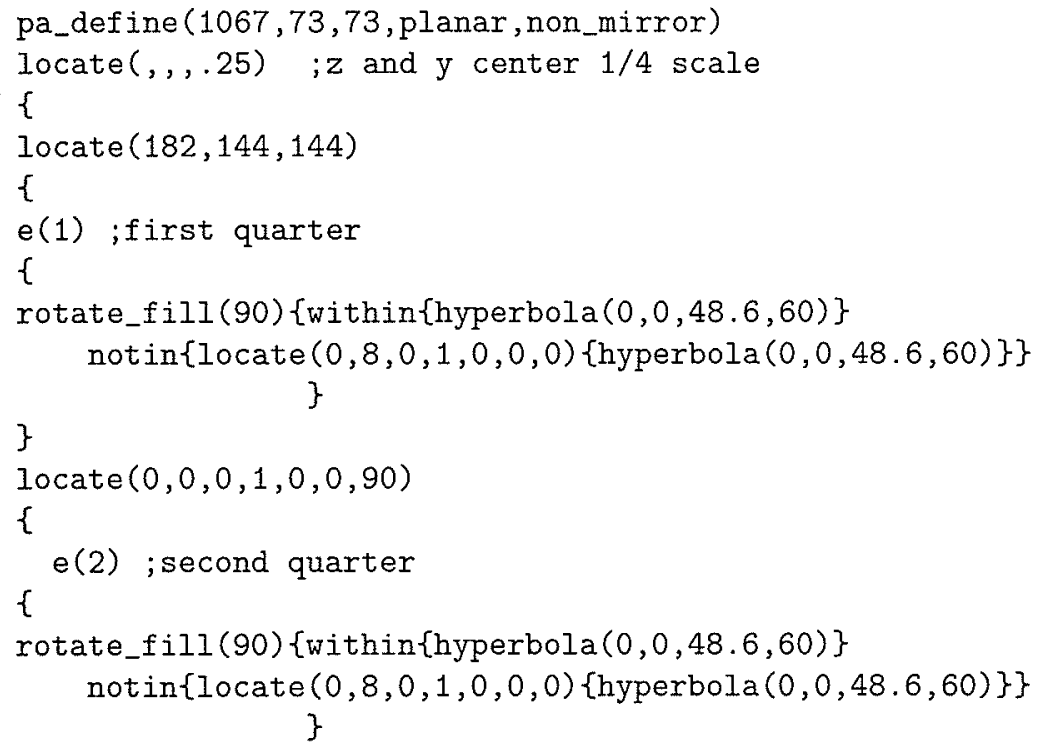




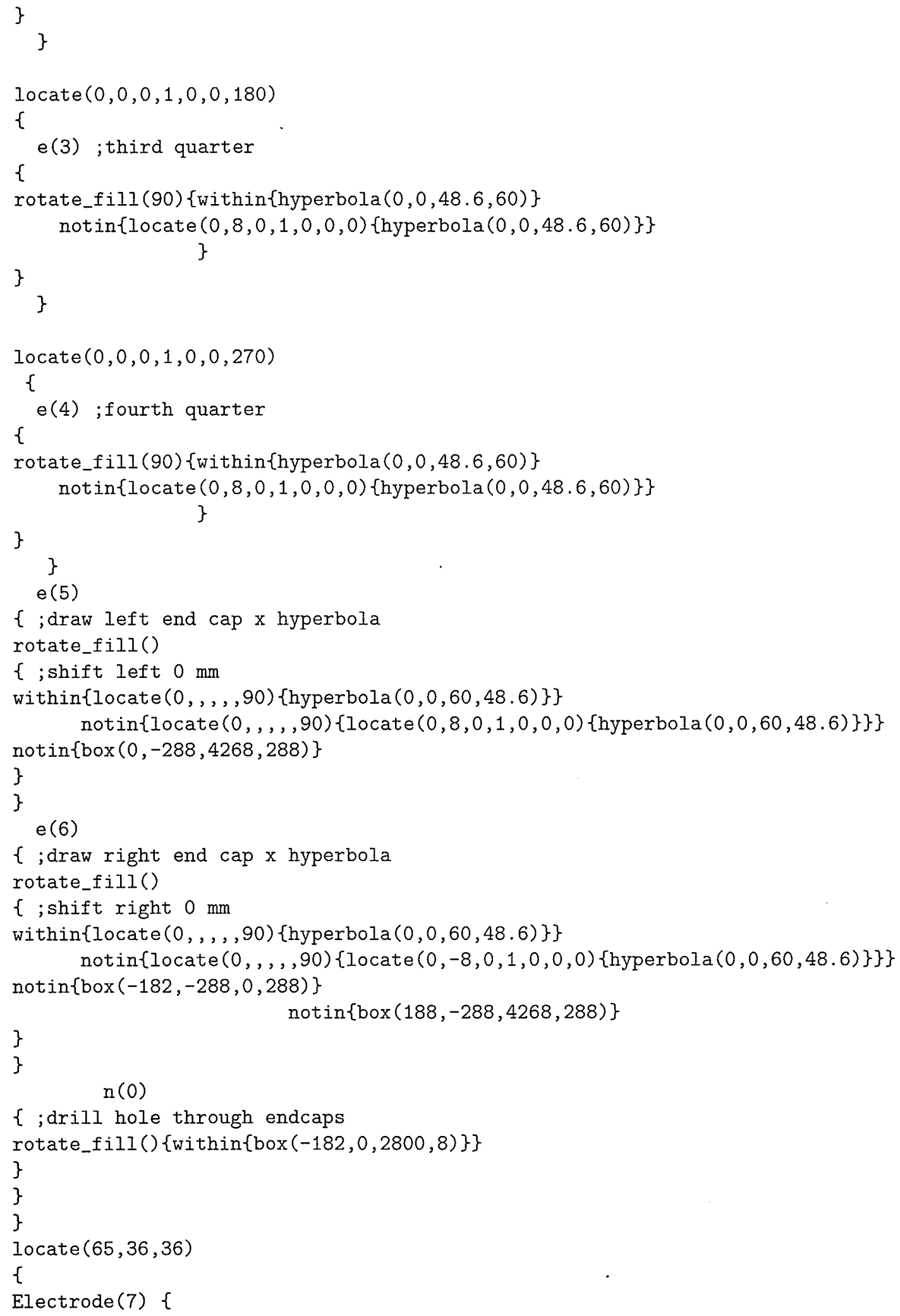




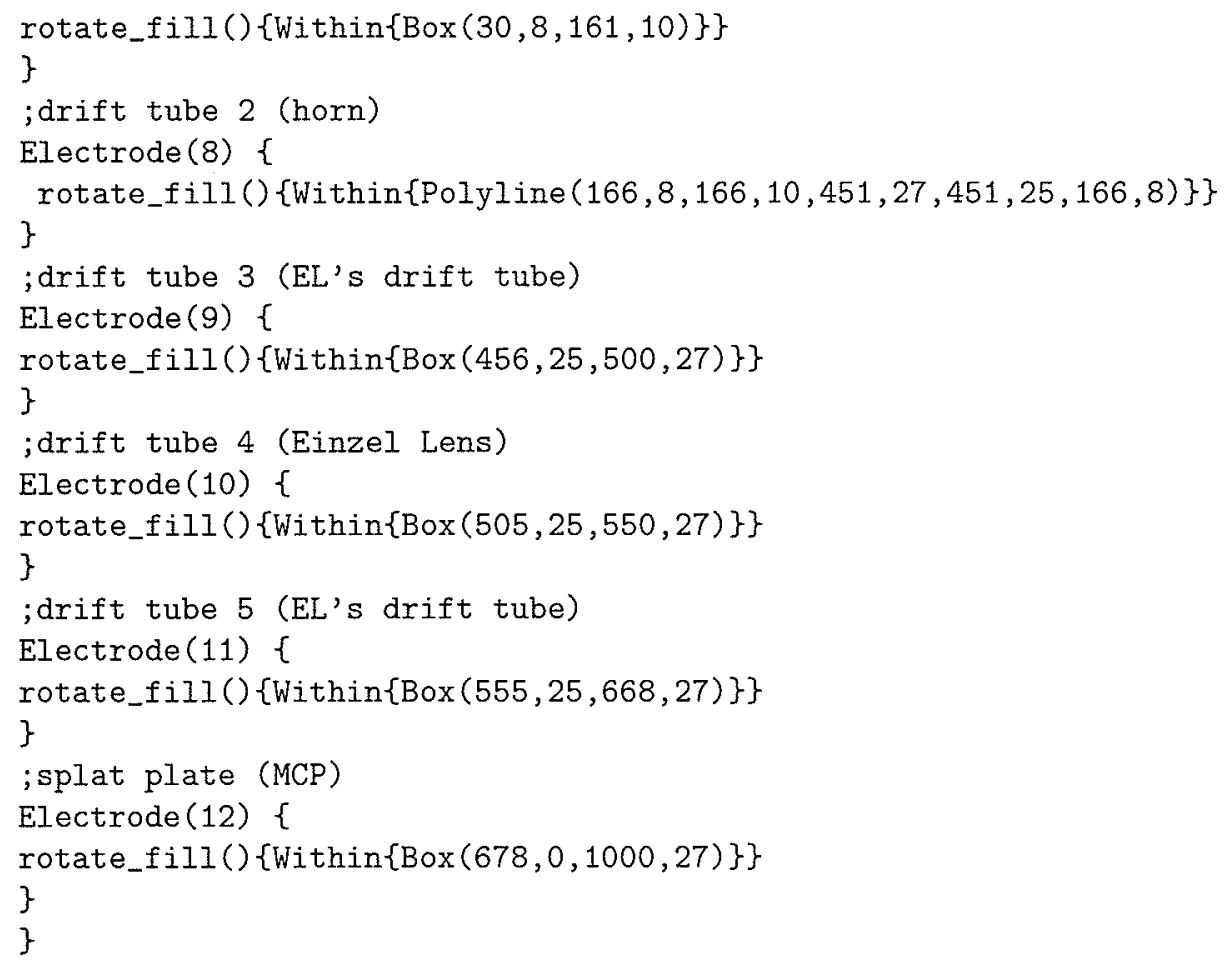

\subsection{Appendix C: User program for simulation of time of flight}

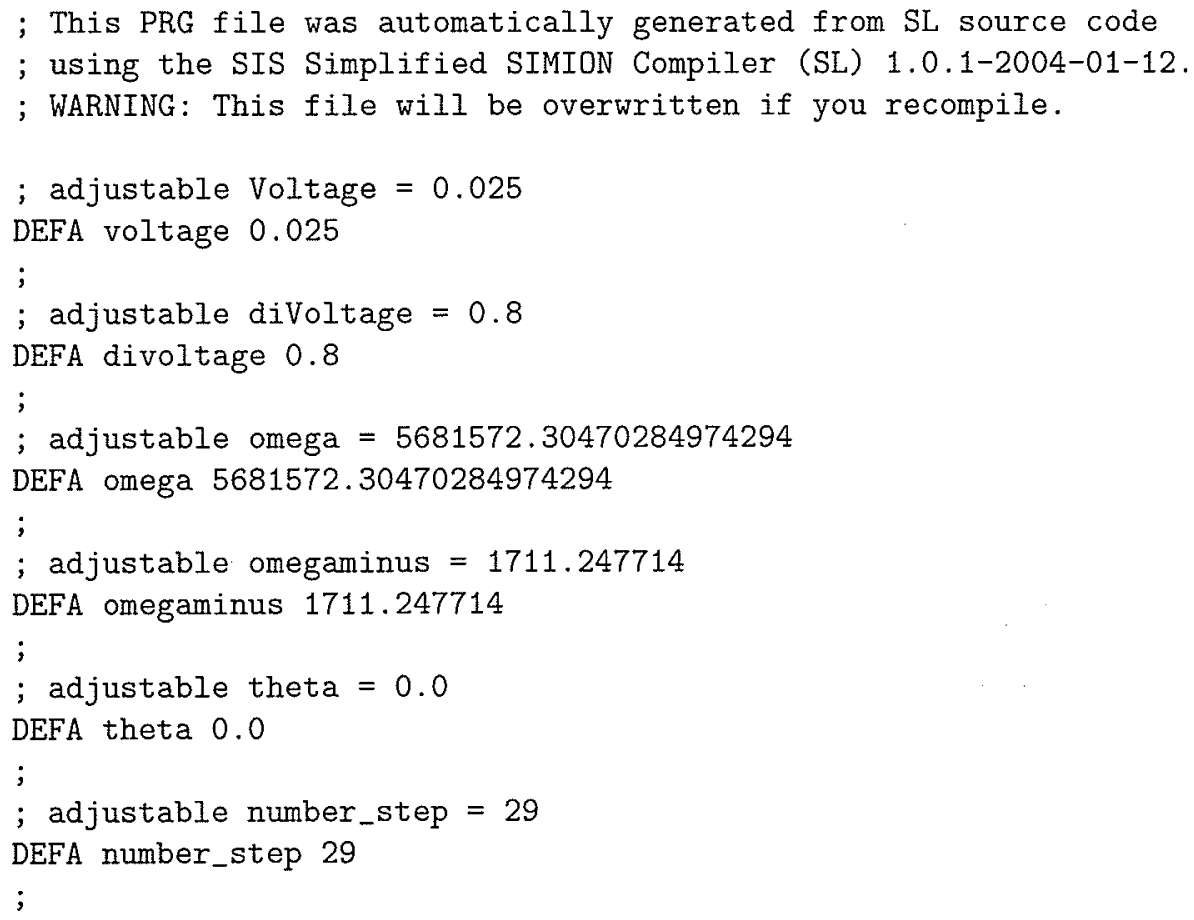




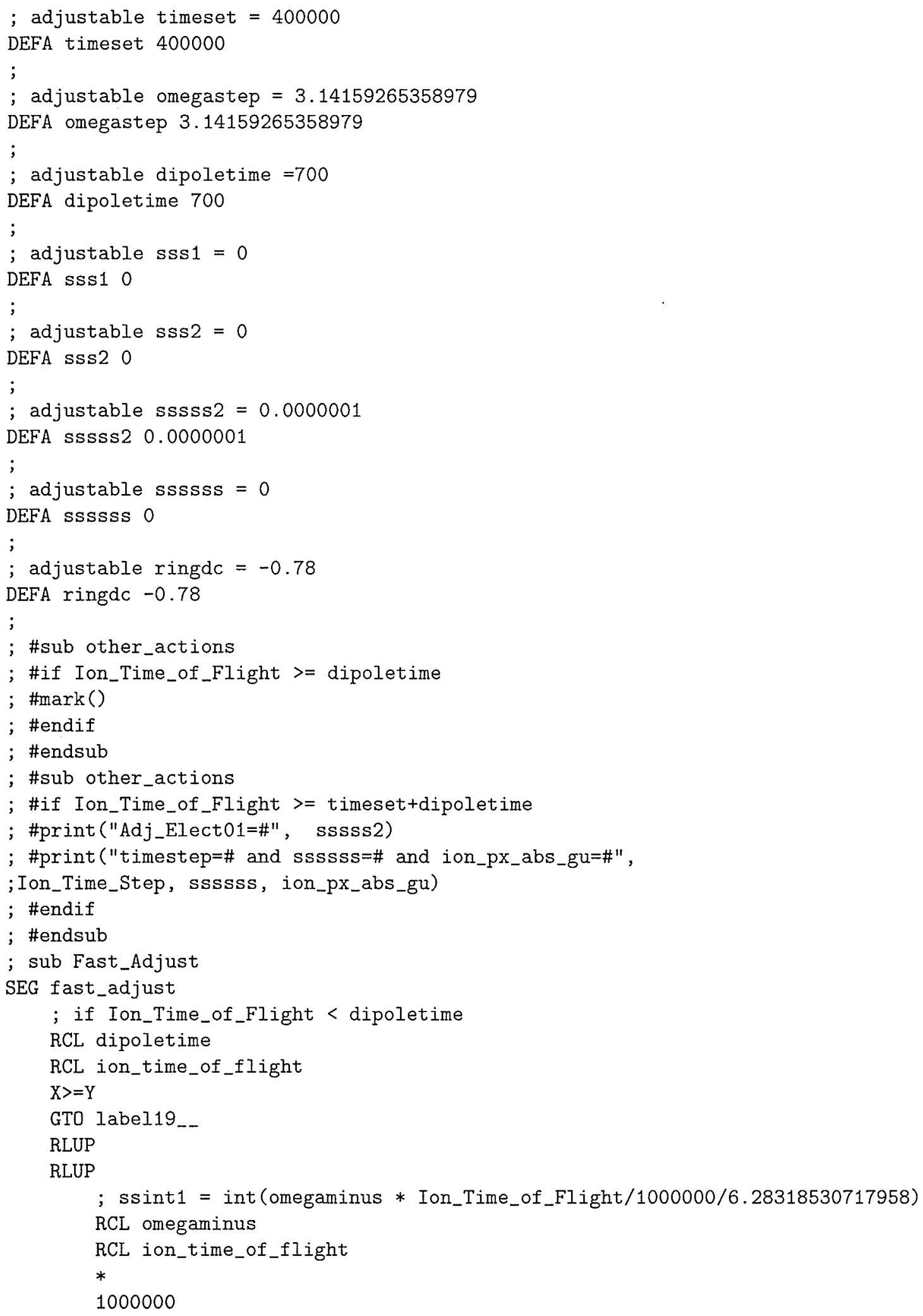




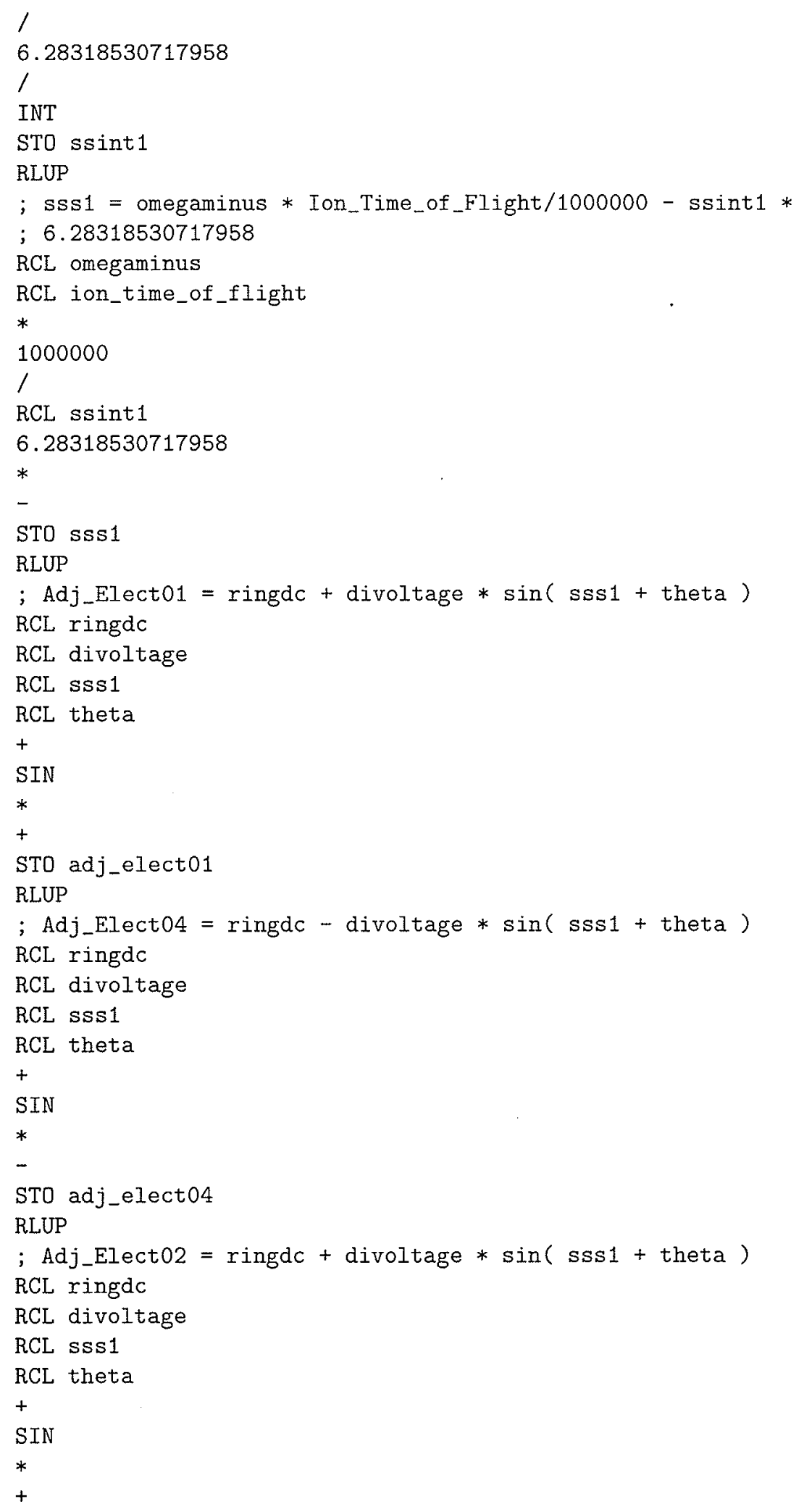




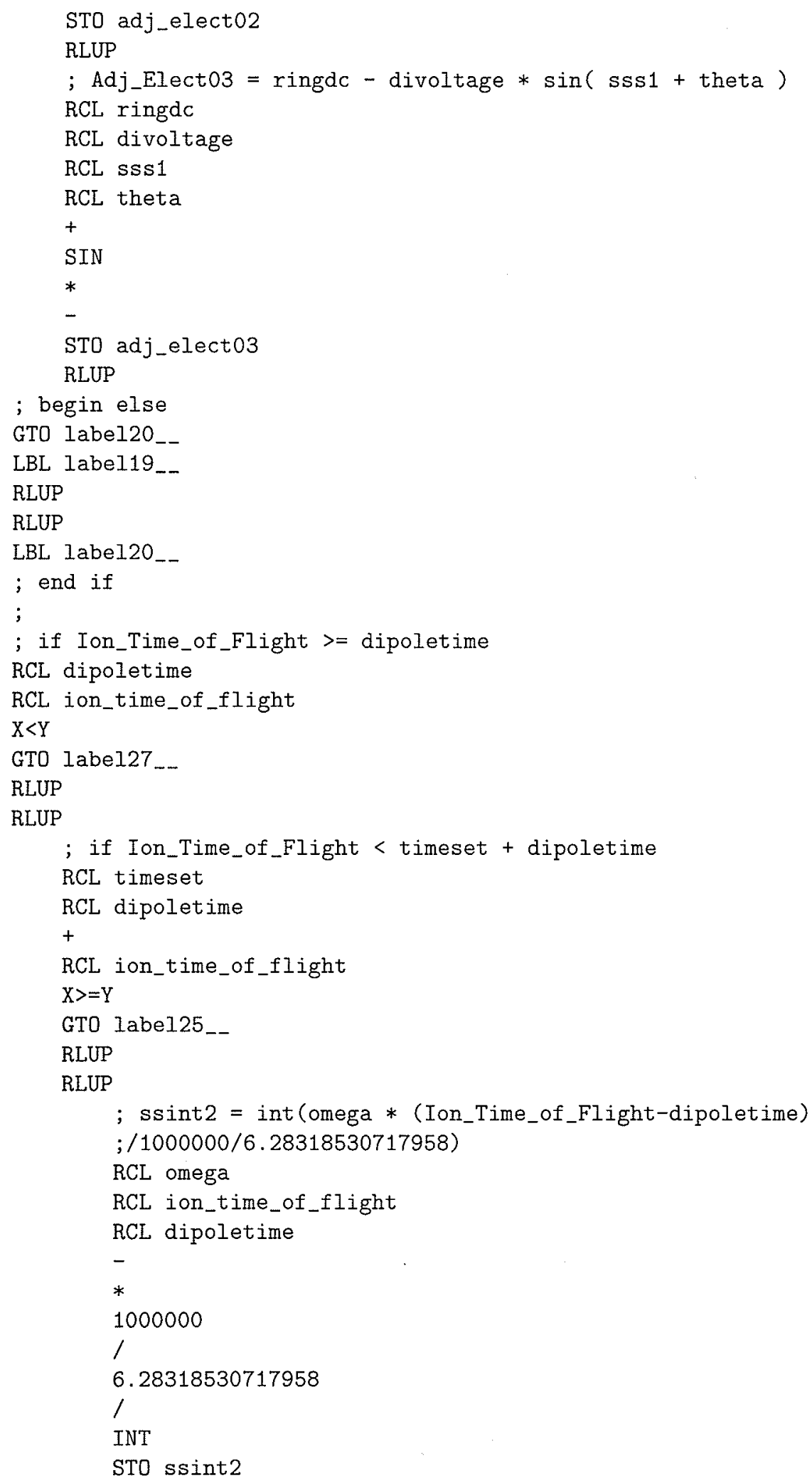




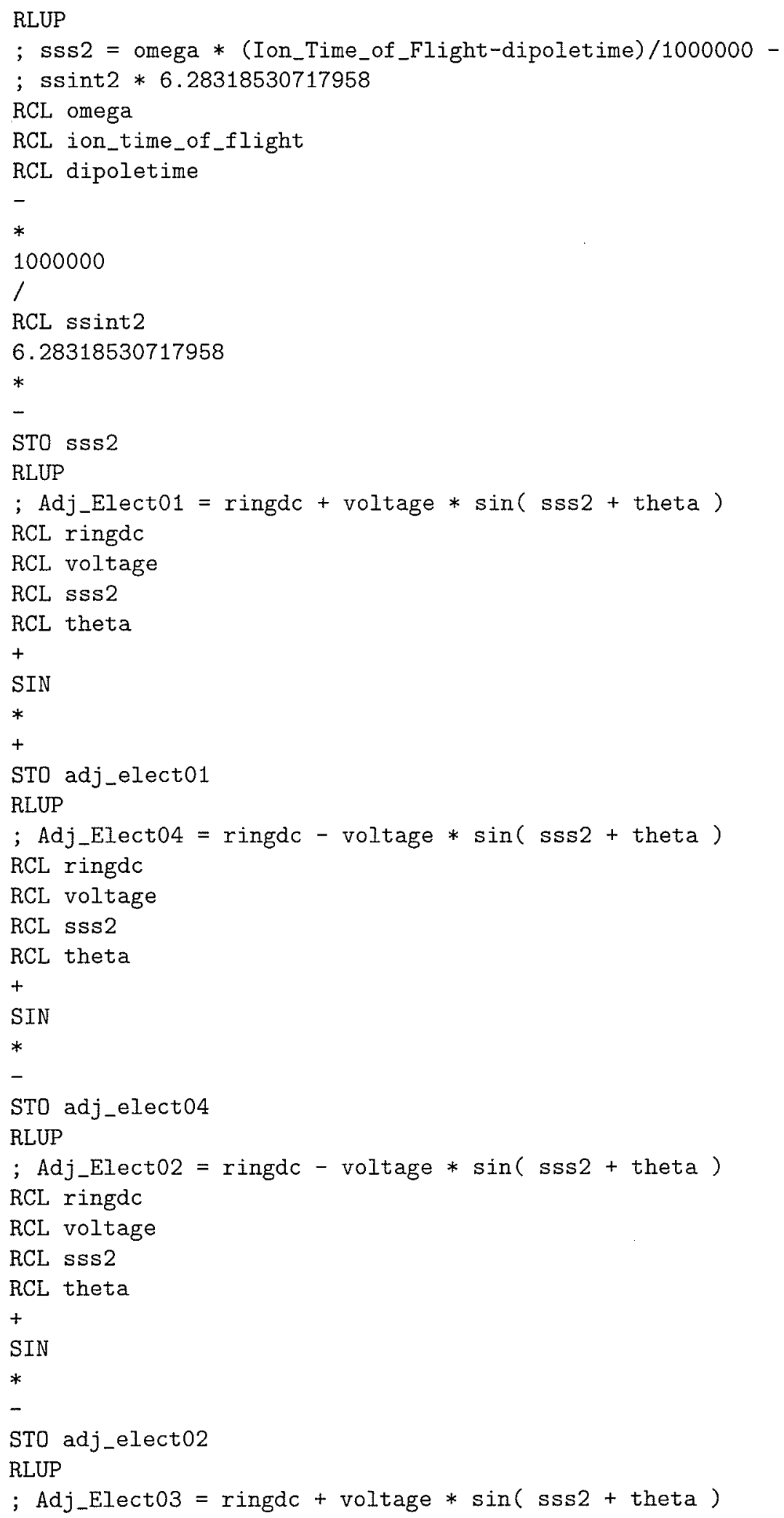




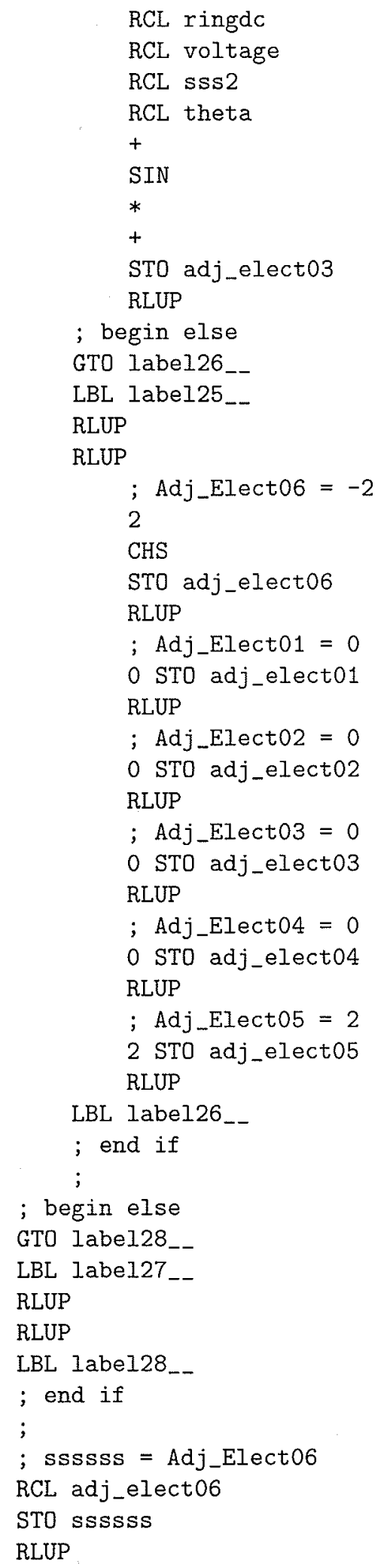




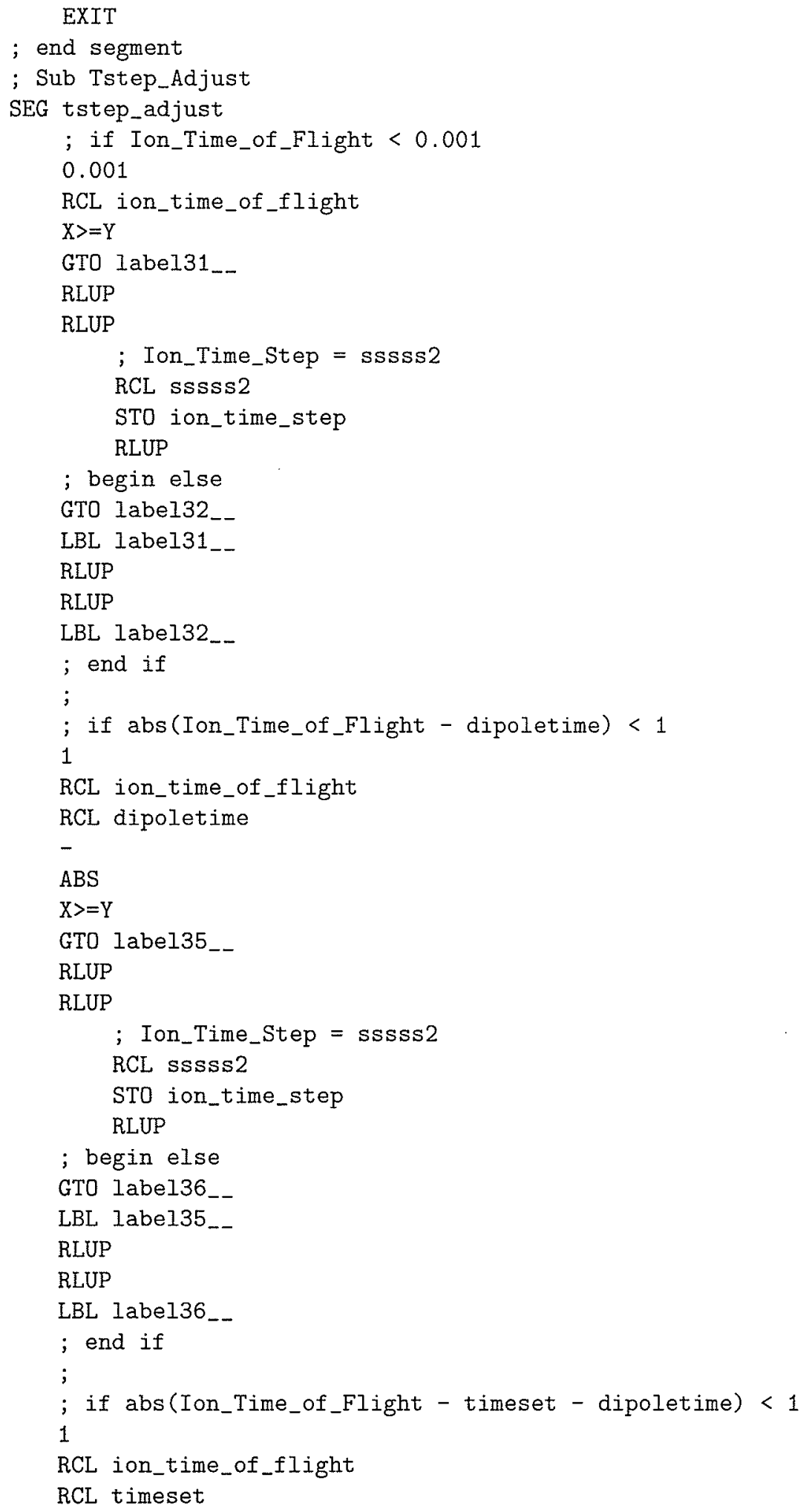


RCL dipoletime

-

ABS

$\mathrm{X}>=\mathrm{Y}$

GT0 labe139_-

RLUP

RLUP

; Ion_Time_Step = sssss2

RCL sssss 2

STO ion_time_step

RLUP

; begin else

GTO label40.

LBL label39...

RLUP

RLUP

LBL label40_-

; end if

;

EXIT

; end segment

; sub Terminate

SEG terminate

; Rerun_Flym = 1

1 STO rerun_flym

RLUP

; omega $=$ omega + omegastep

RCL omega

RCL omegastep

$+$

STO omega

RLUP

; number_step $=$ number_step -1

RCL number_step

1

STO number_step

RLUP

; print ("omega=\# and sss1=\# and sss2=\# and sssss2=\#", ; omega-omegastep, sss1, sss2, sssss2)

RCL sssss 2

RCL sss2

RCL sss 1

RCL omega

RCL omegastep

MESS ; omega=\# and $\operatorname{sss} 1=\#$ and $\operatorname{sss} 2=\#$ and $\operatorname{sssss} 2=\#$

RLUP

RLUP

RLUP 


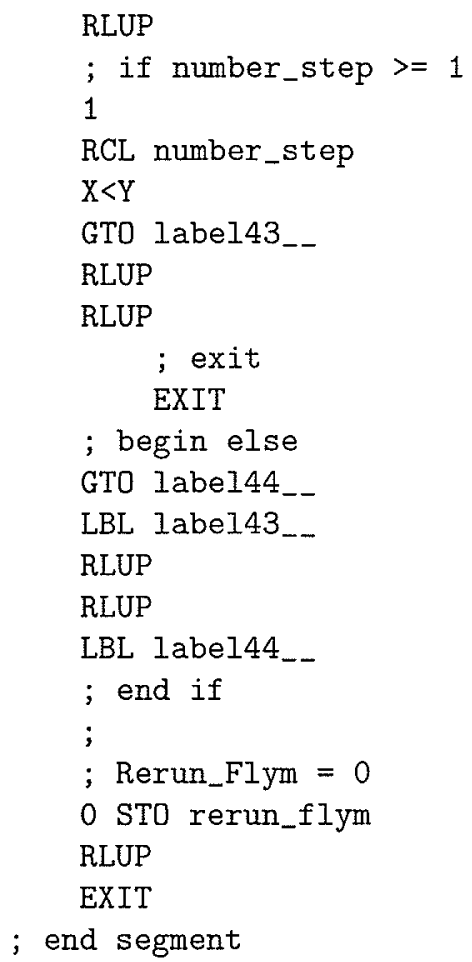

\subsection{Appendix D: User program for loading magnetic fields into SIMION}

The ion_bfieldx_gu, ion_bfieldy_gu, ion_bfieldz_gu are respectively $x, y$, and $z$ components of magnetic field, they can be set by .prg file which is a user program in SIMION 7.0 shown below.

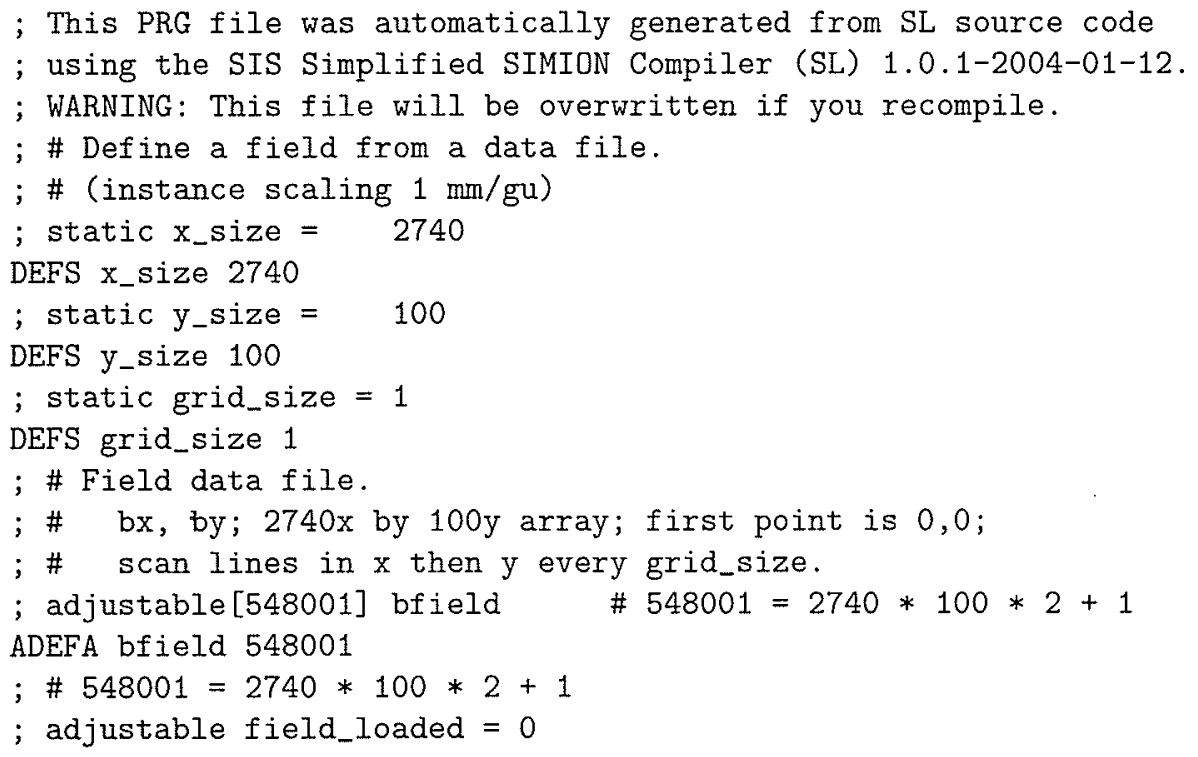




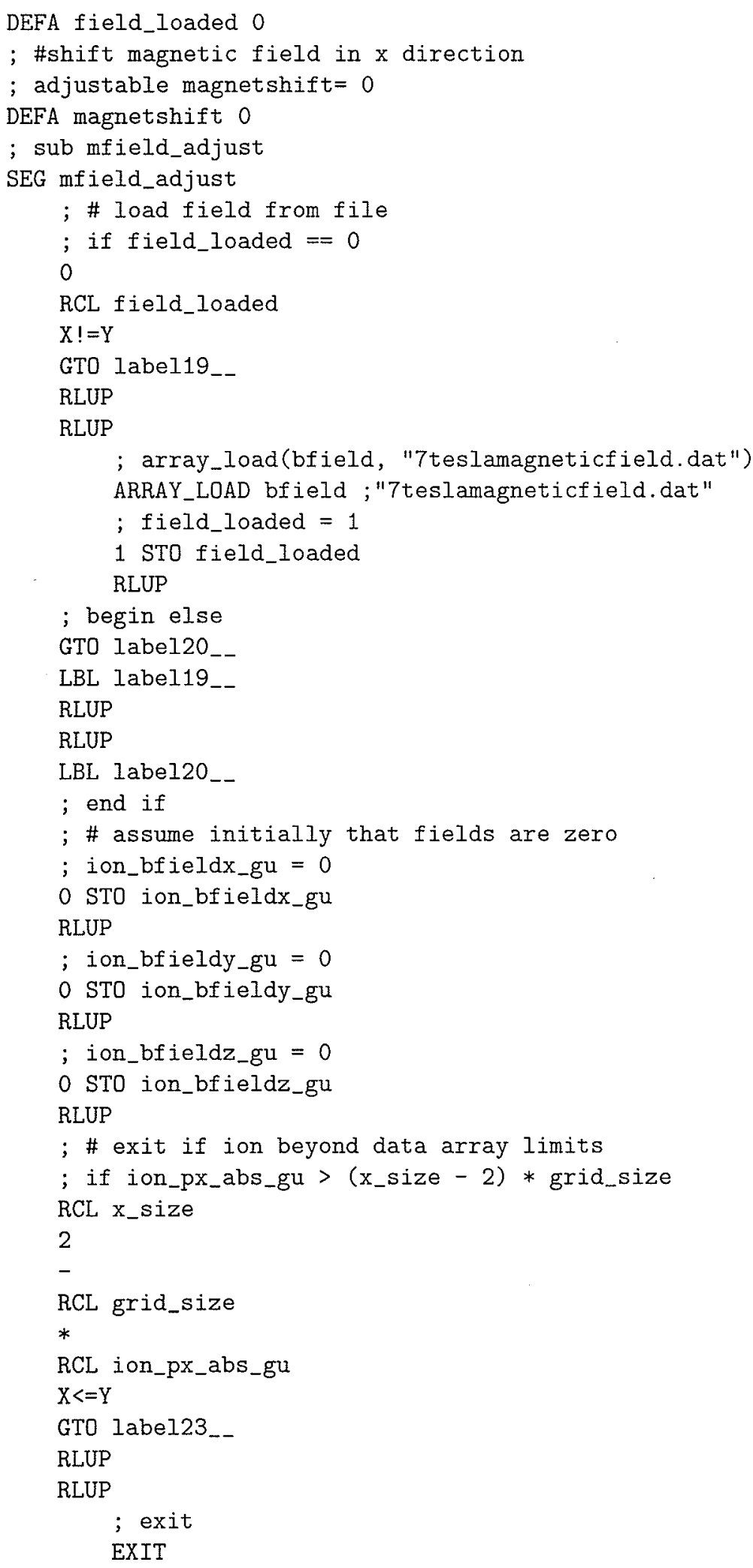




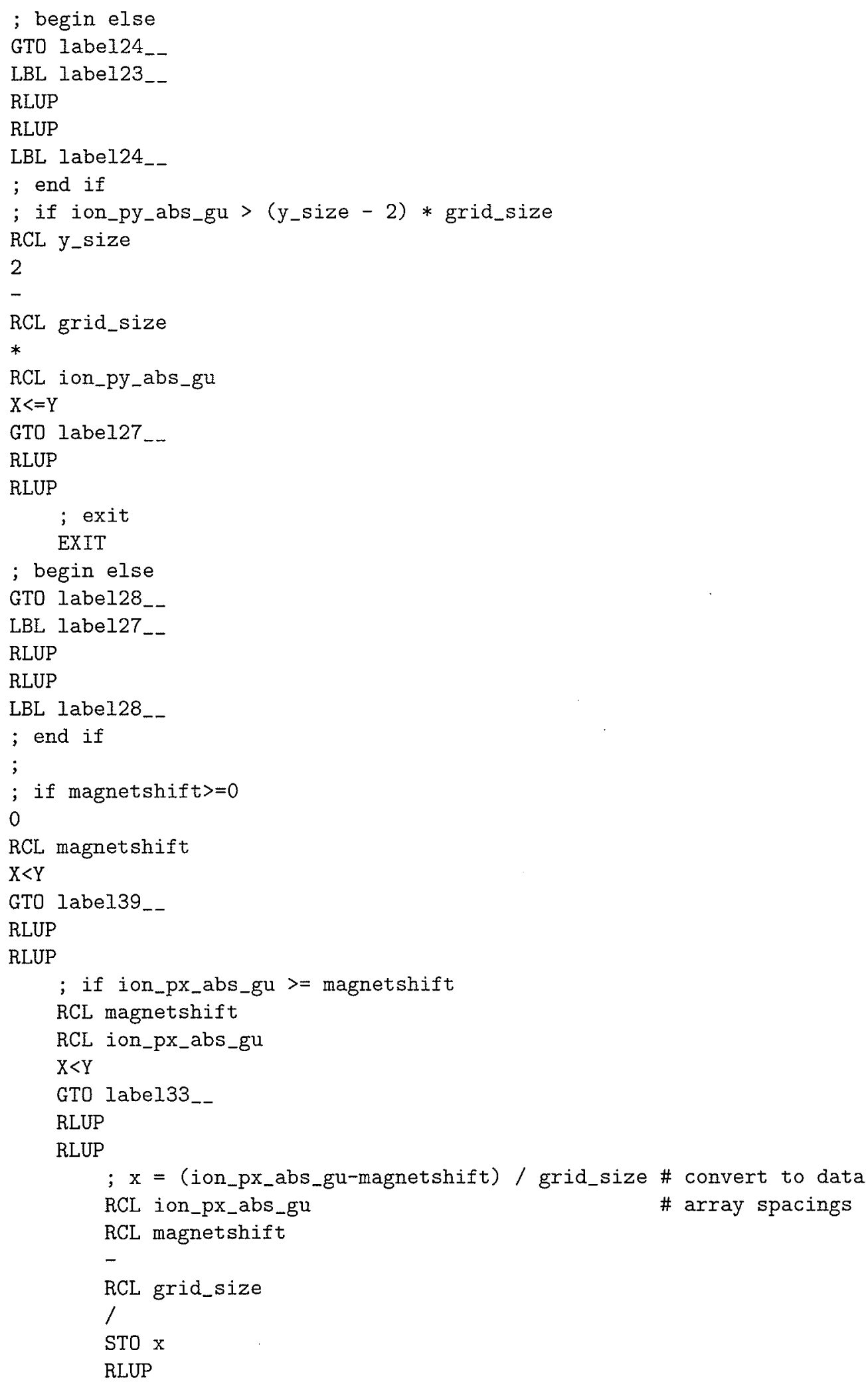




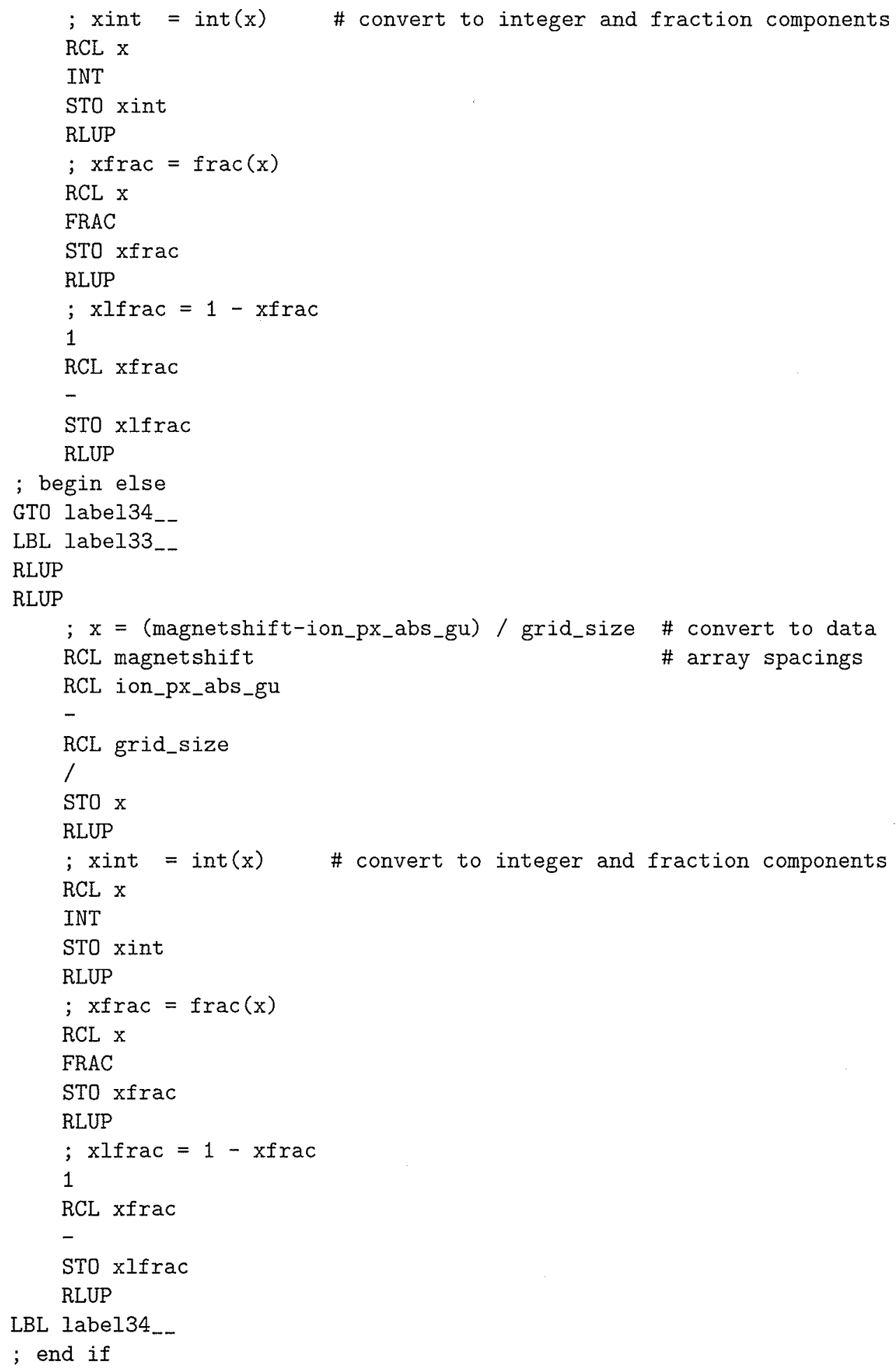




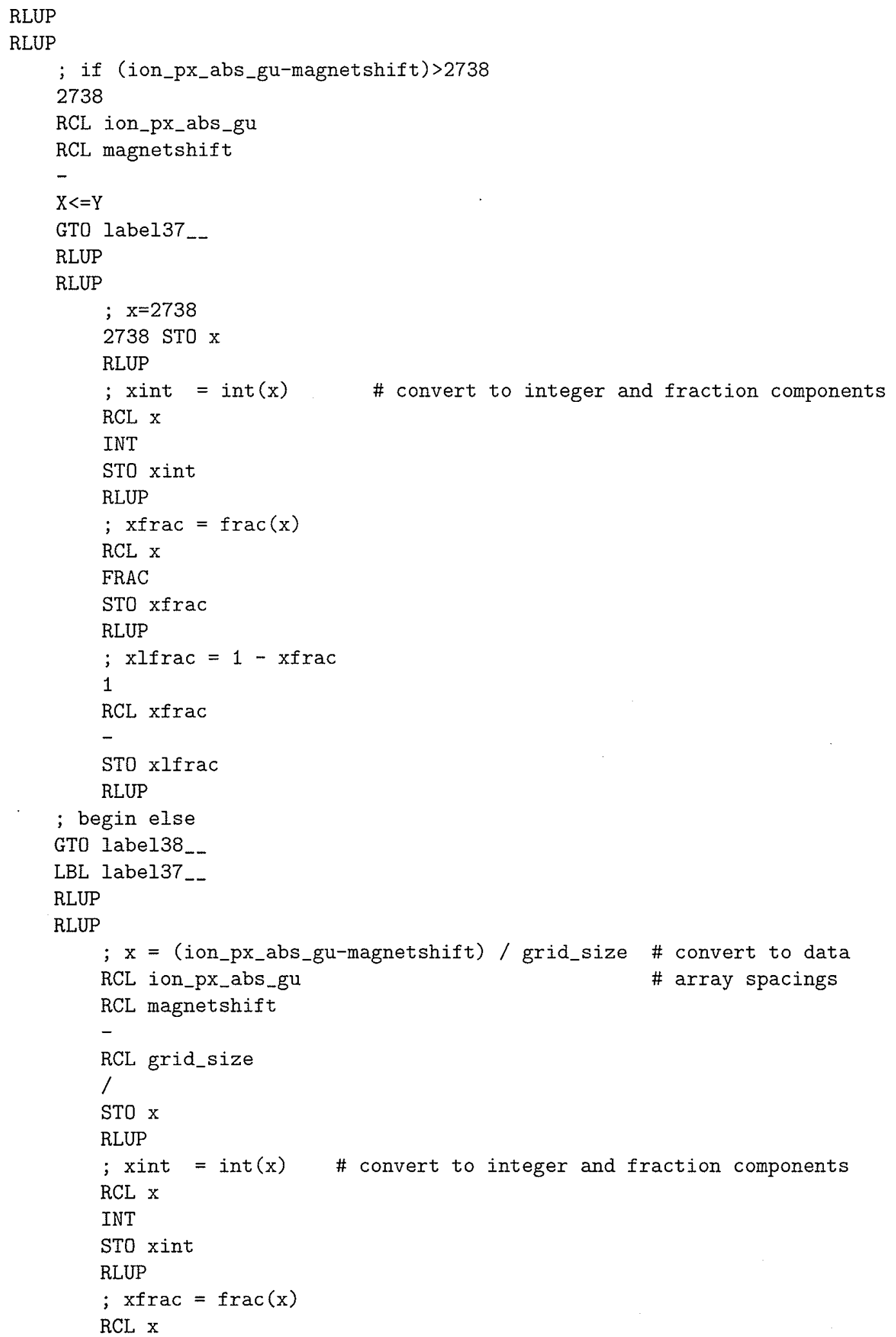




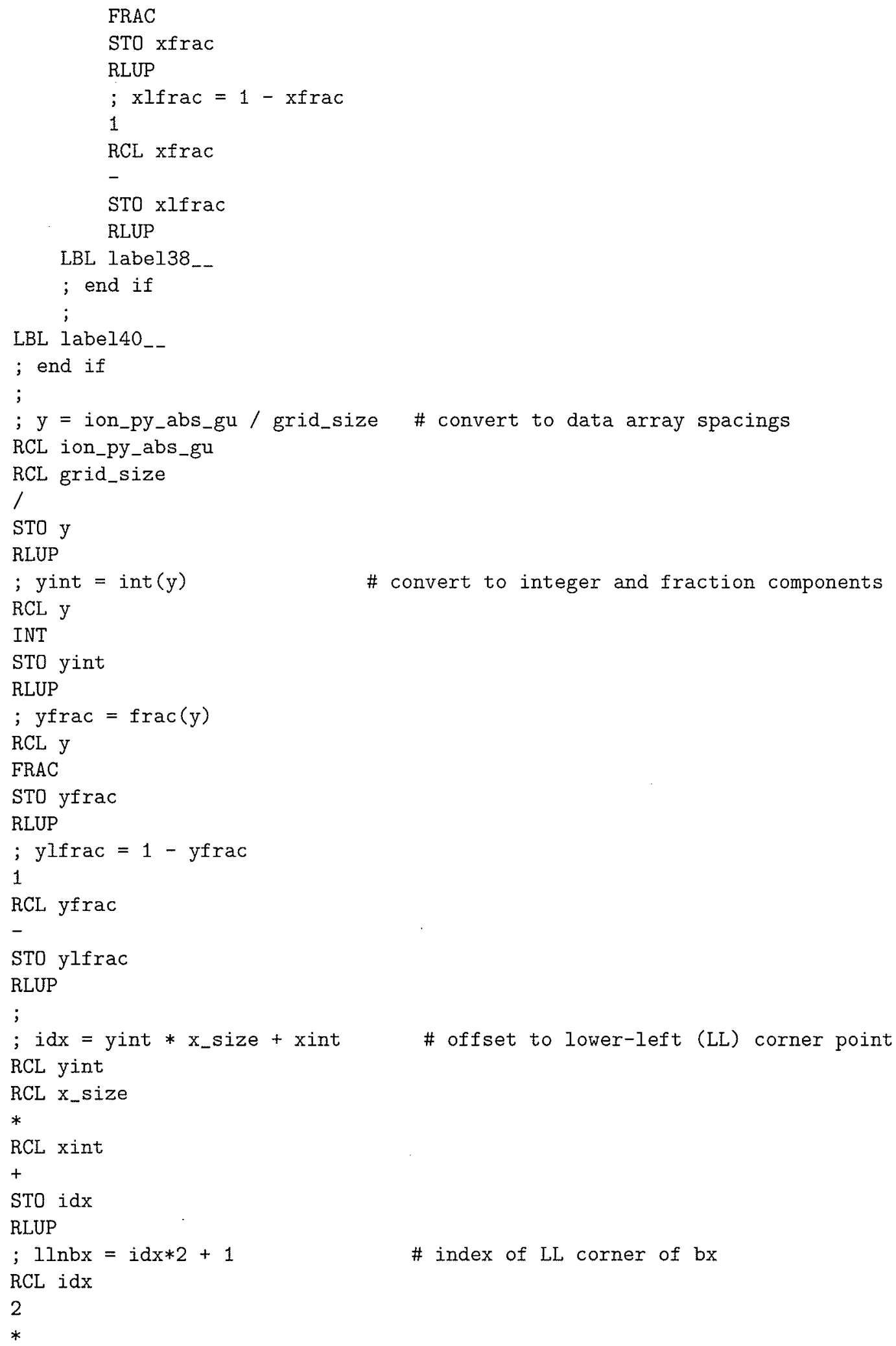


1

$+$

STO $1 \operatorname{lnbx}$

RLUP

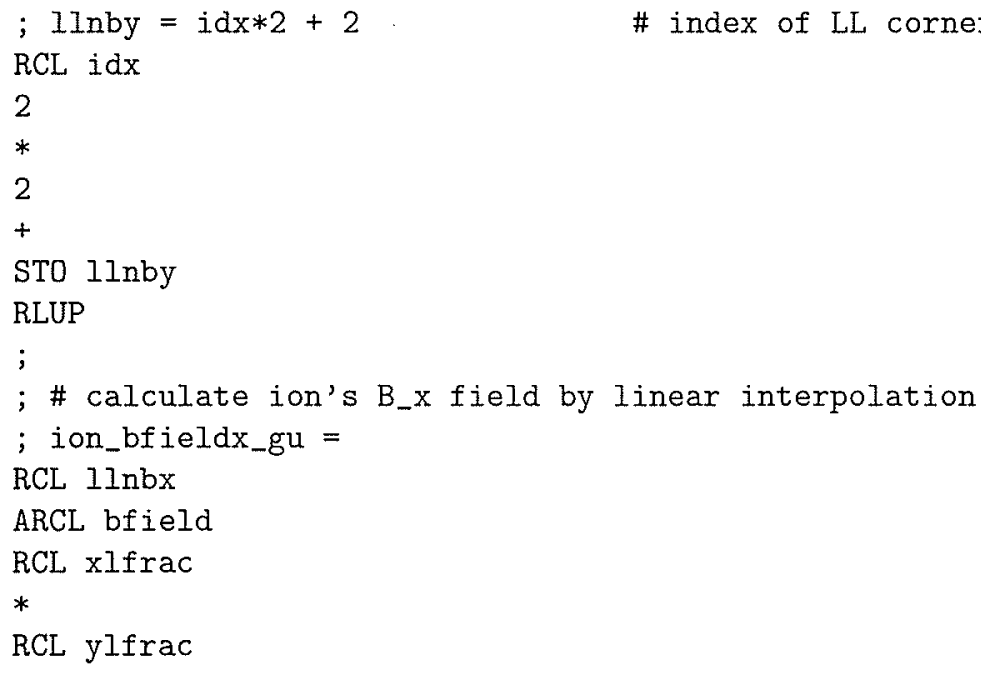




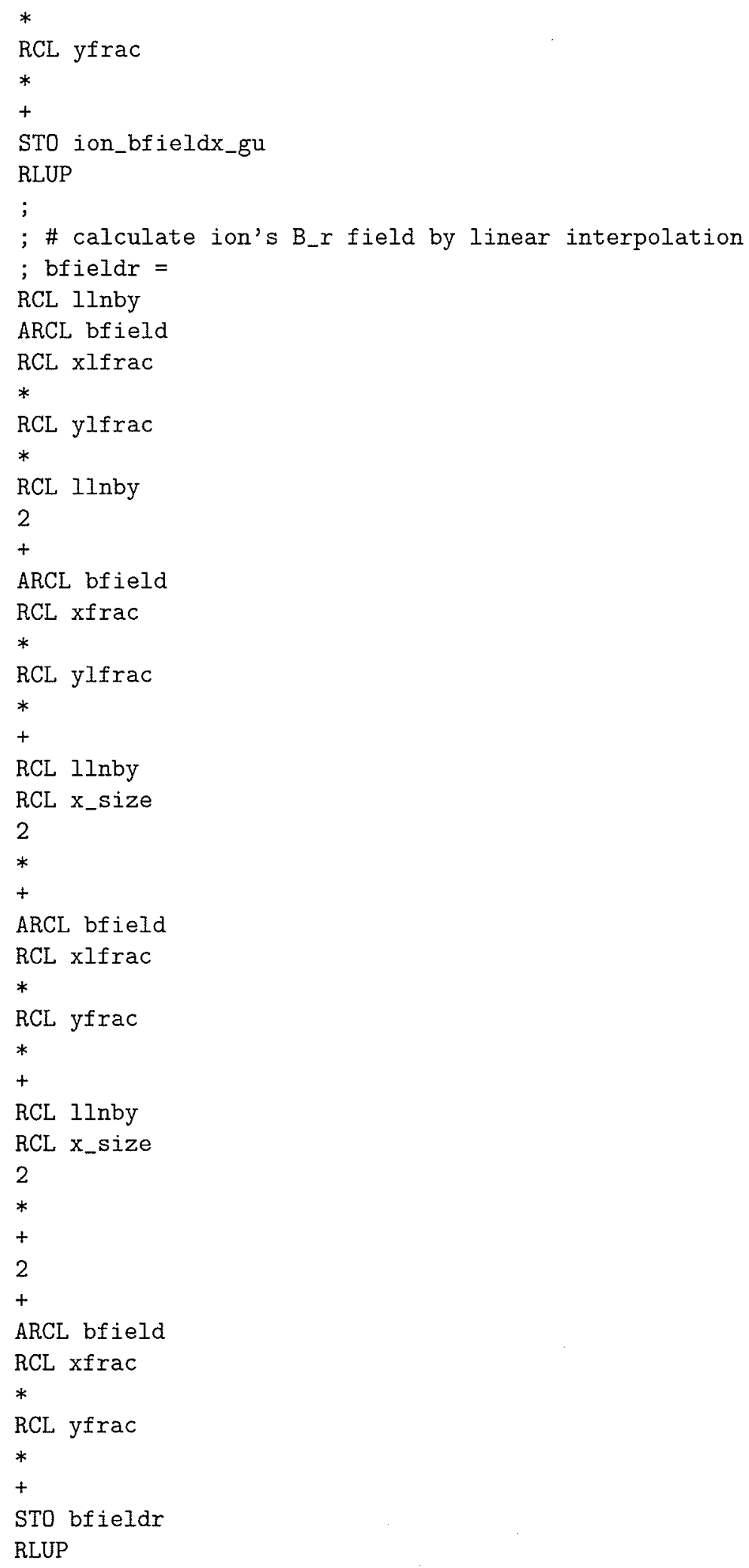




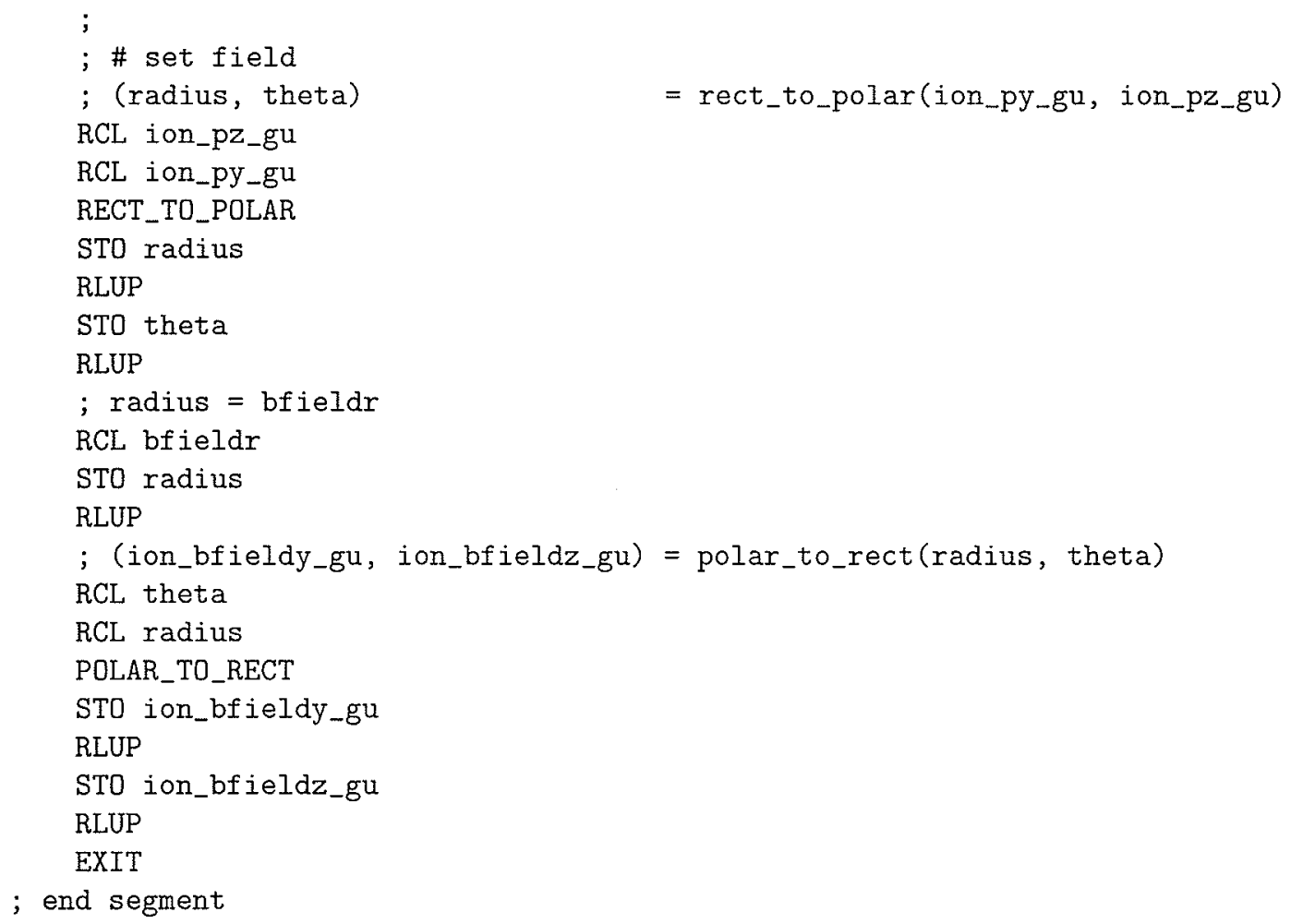

\subsection{Appendix E: Ion or proton injection geometry}

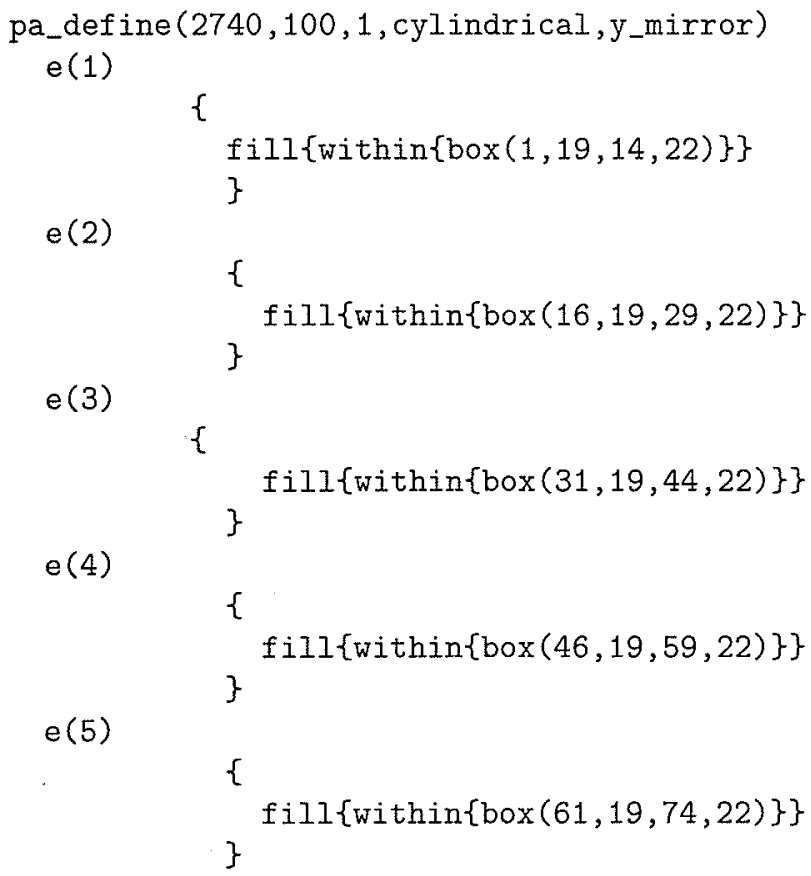




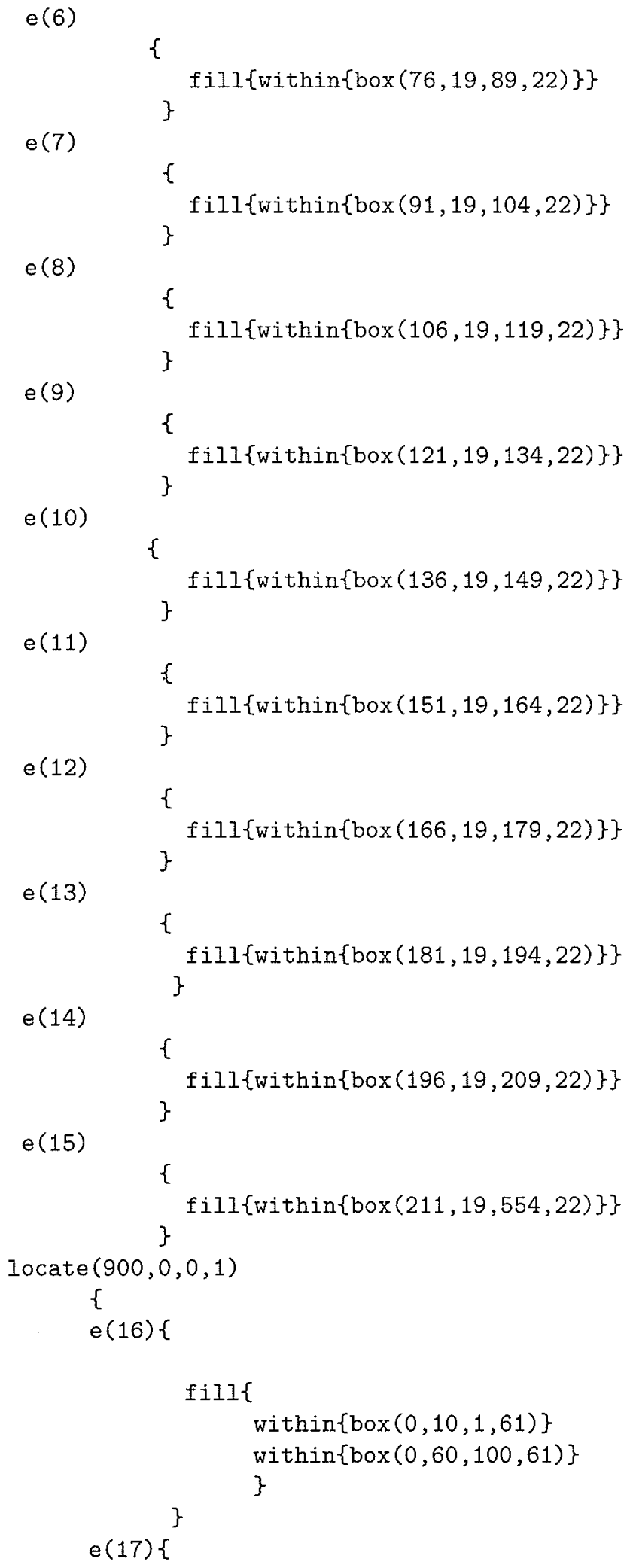




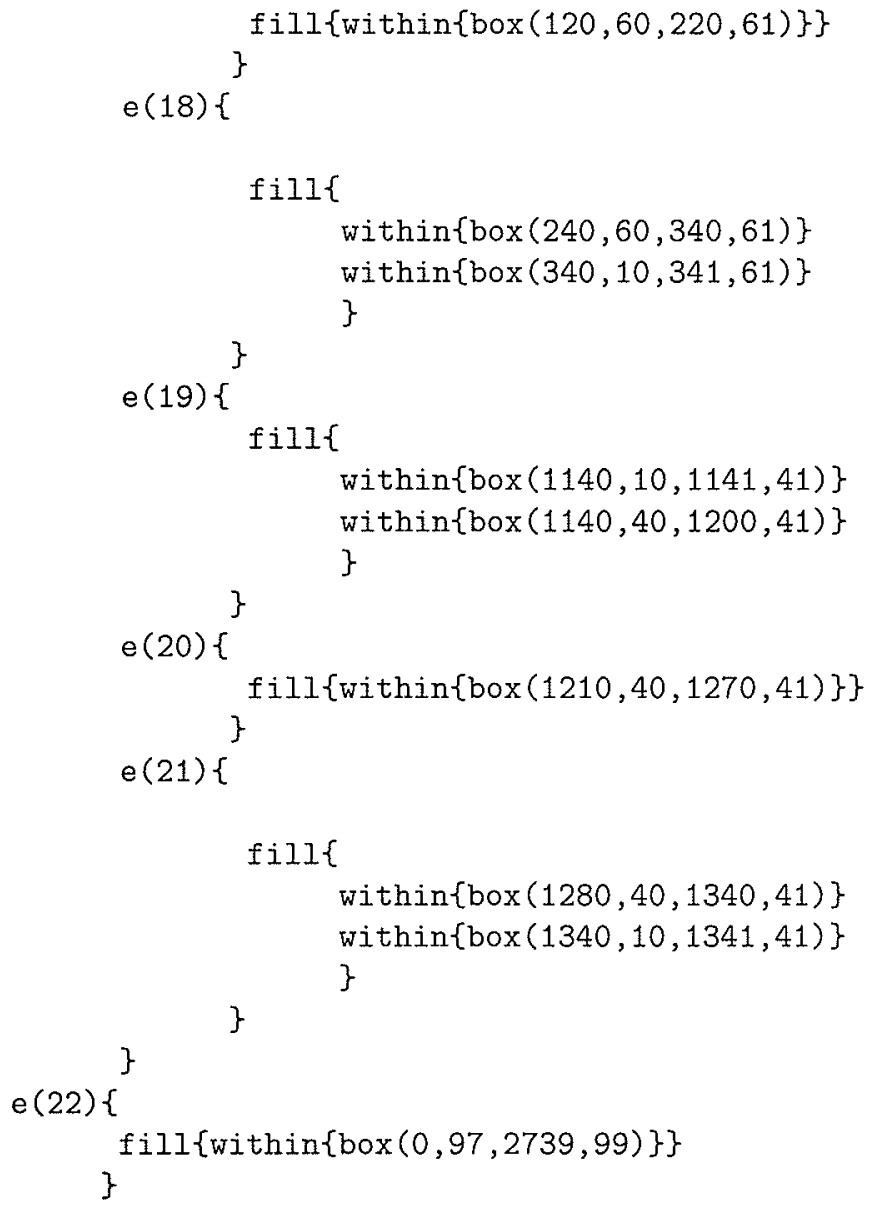

\subsection{Appendix F: Ion source}

We set up an ion system at TRIUMF in Vancouver, and used it to produce the test beam for the electron beam ion trap and the cooler trap. The ion source is shown in Figure 7.1. The ion beam emittance is measured by an emittance meter [118] which is shown in Figure 7.2 and 7.3. It mainly consists of a pair of electric deflection plates, a front slit and a rear slit. Ions enter the electric field region from the front slit and 
go through the rear slit. The initial angle is given by

$$
\theta \approx \frac{V}{\phi} \frac{L}{4 g},
$$

where $V$ is the deflection voltage, $q \phi$ gives the ion energy determined by the ion source floating voltage, and $L(2.75$ in $)$ and $g(0.16$ inch $)$ are the length and distance of the deflection planes respectively.

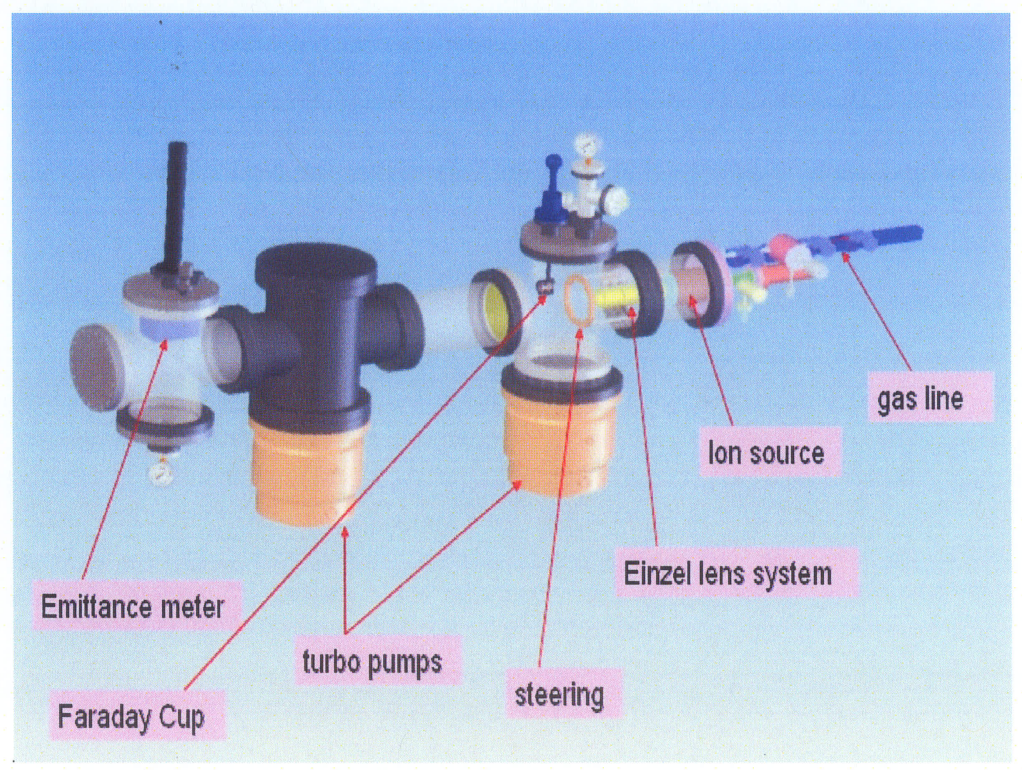

Figure 7.1: Ion source.

The angular resolution is given by

$$
\triangle \theta=\frac{ \pm s}{D},
$$

where $s$ is the width of the front and rear slits, which can be changed from 0.001-0.01 inch, and $D$ is the distance between the front and rear slits. From Eq. 7.22, the angular resolution that can be reached is $0.35 \mathrm{mrad}$. 


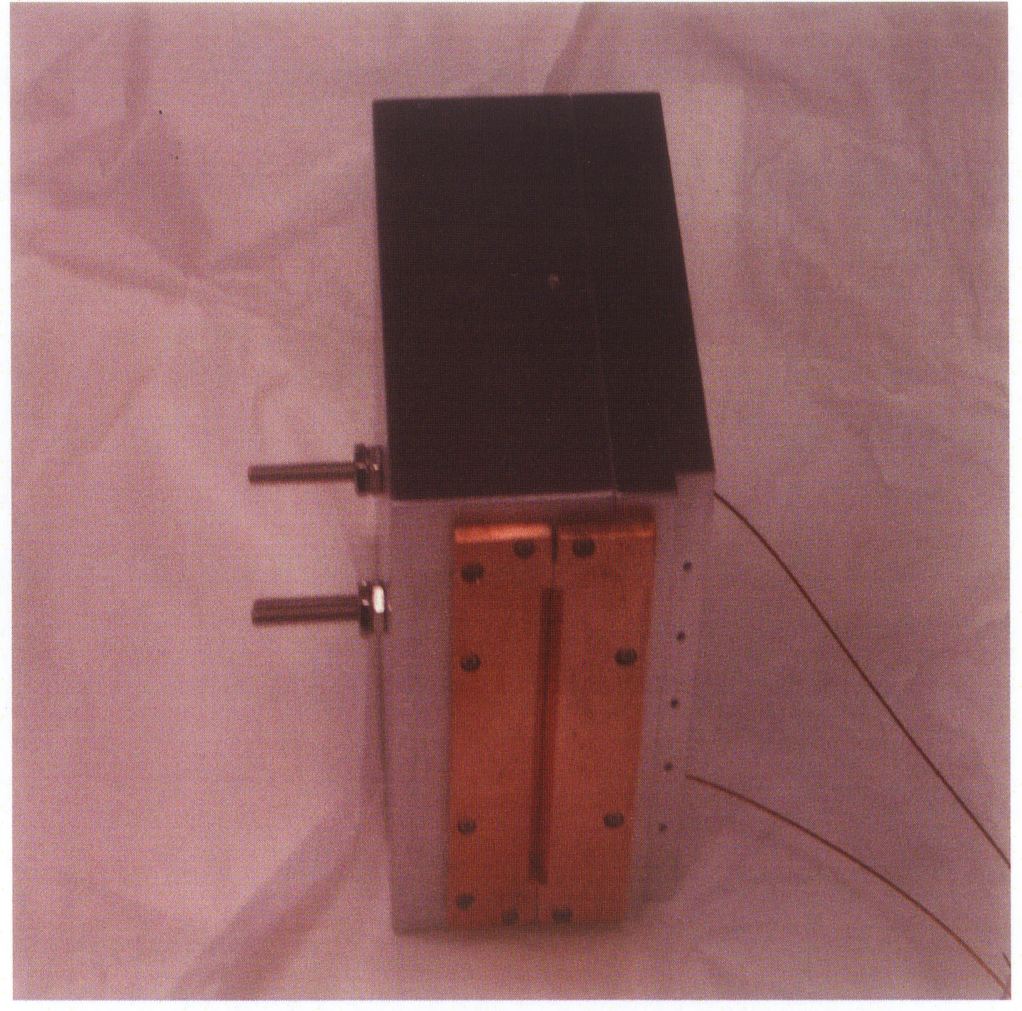

Figure 7.2: Emittance meter. 


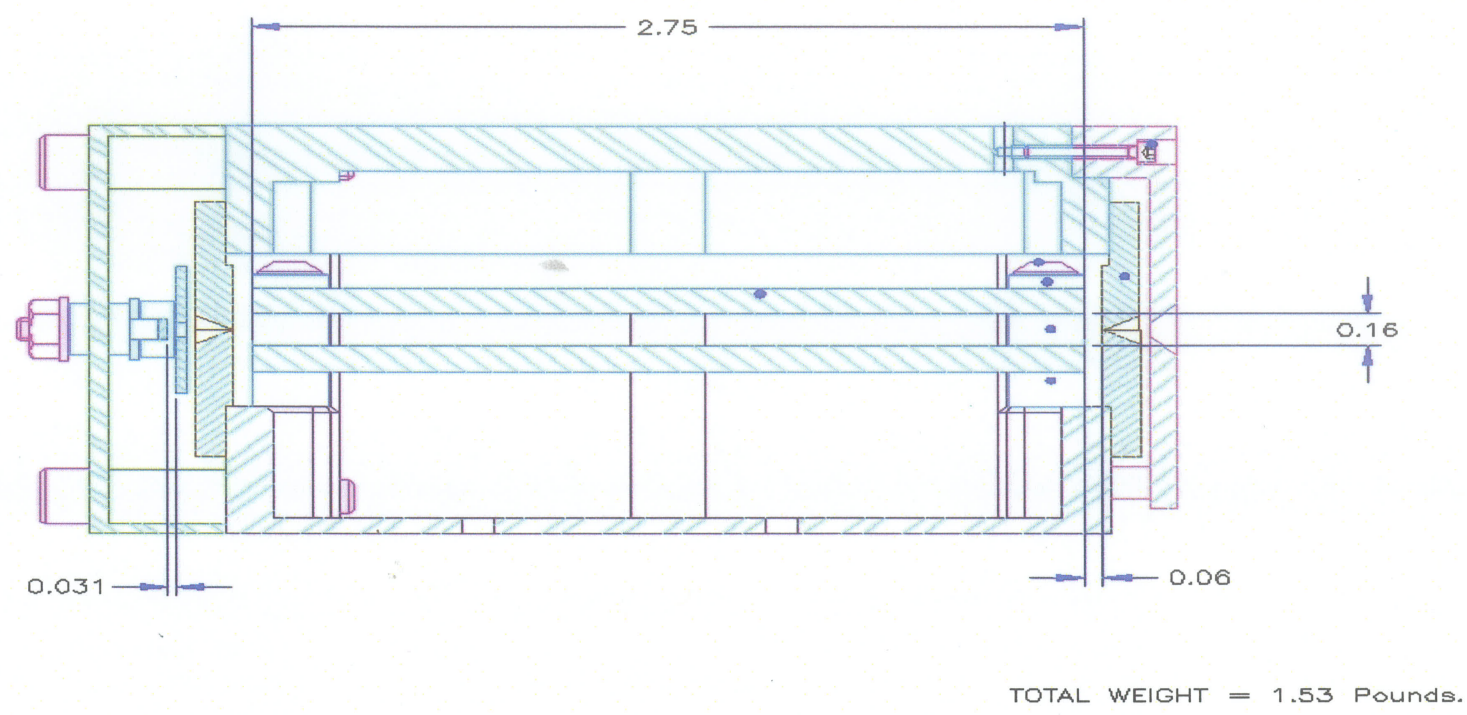

Figure 7.3: Mechanical drawing of the emittance meter.

Figure 7.4 shows the xenon beam emittance measurement result. Initially, the vacuum was $1.7 \times 10^{-7}$ Torr. Once xenon gas was let in, the vacuum rose to $8.2 \times 10^{-7}$ Torr.

Figure 7.4 shows the hydrogen beam emittance measurement result, the vacuum is $4.5 \times 10^{-7}$ Torr near ion source before gas was let in. Once hydrogen was admitted, the vacuum became worse. The vacuum for this scan was about $1.9 \times 10^{-5}$ Torr.

The measured beam emittances were less than $10 \pi \mathrm{mm}$ mrad, sufficient for use with the EBIT and the cooler trap. 

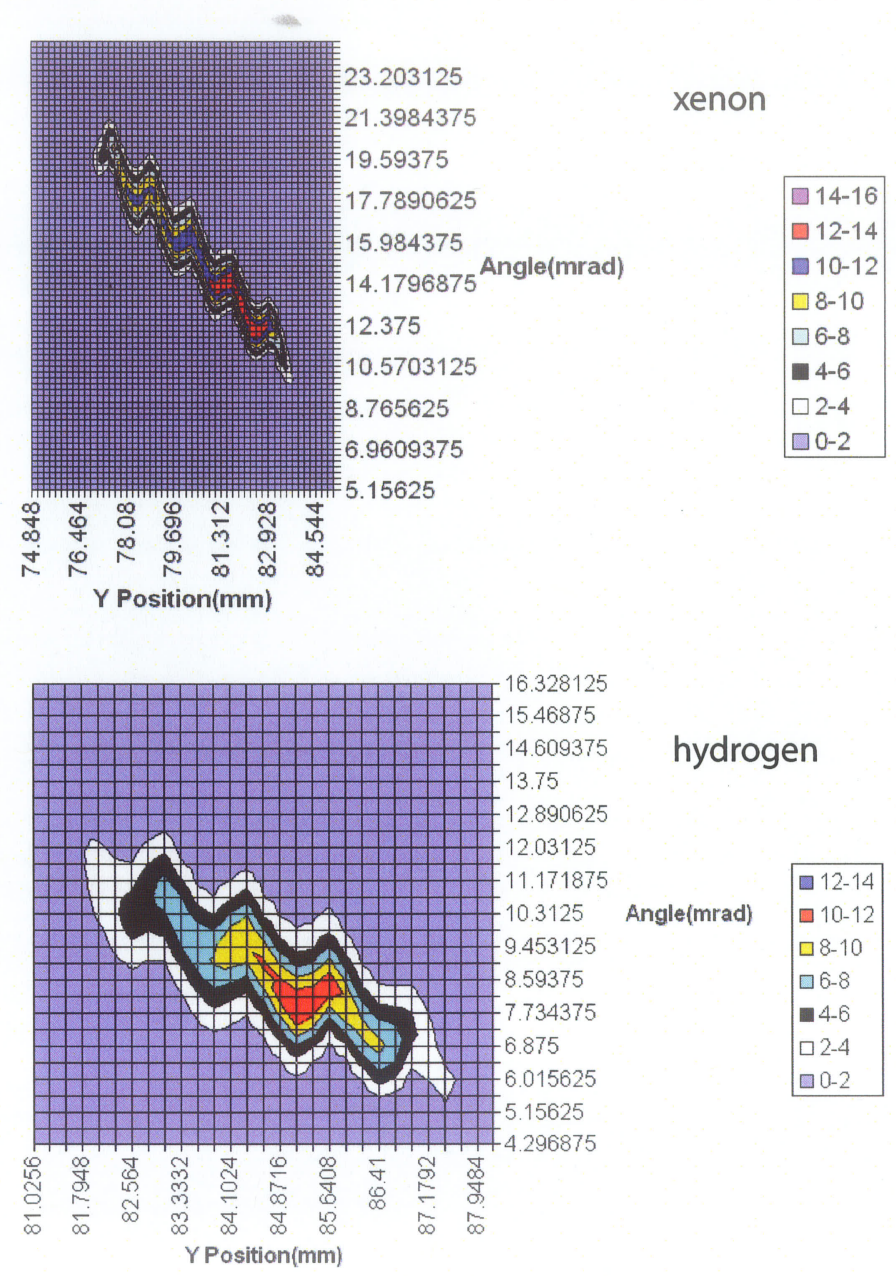

Figure 7.4: Xe beam emittance and hydrogen beam emittance, the unit of measured current is $\mathrm{nA}$. 


\section{BIBLIOGRAPHY}

[1] D. Lunney, J. M. Pearson, C. Thibault, Recent trends in the determination of nuclear masses, Rev. Mod. Phys. 75, 1021(2003).

[2] C. F. von Weizsäcker, Zur Theorie der Kernmassen. Z. Phys. 96, 431(1935).

[3] H. A. Bethe and R. F. Bacher, Nuclear Physics A. Stationary states of nuclei, Revs. Mod. Phys. 8, 162(1936).

[4] W. D. Myers and W. J. Swiatecki, Nuclear masses and deformations, Nucl. Phys. $81,1(1966)$

[5] D. Vautherin and D. M. Brink, Hartree-Fock calculations with Skyrme's interaction. I. Spherical Nuclei, Phys. Rev. C 5, 626(1972).

[6] E. Chabanat, P. Bonche, P. Haensel, J. Meyer, and R. Schaeffer, A Skyrme parametrization from subnuclear to neutron star densities, Nucl. Phys. A 627, 710(1997)

[7] E. Chabanat, P. Bonche, P. Haensel, J. Meyer, and R. Schaeffer, A Skyrme parametrization from subnuclear to neutron star densities Part II. Nuclei far from stabilities, Nucl. Phys. A 635, 231(1998). 
[8] S. Goriely, M. Samyn, P.-H. Heenen, J. M. Pearson, and F. Tondeur, HartreeFock mass formulas and extrapolation to new mass data, Phys. Rev. C 66, 024326 (2002).

[9] J. Dobaczewski, W. Nazarewicz, T. R. Werner, J. F. Berger, C. R. Chinn, and J. Dechargé, Mean-field description of ground-state properties of drip-line nuclei: Pairing and continuum effects, Phys. Rev. C 53, 2809(1996).

[10] F. Tondeur, S. Goriely, J. M. Pearson, and M. Onsi, Towards a Hartree-Fock mass formula, Phys. Rev. C 62, 024308 (2000).

[11] W. Satula, D. J. Dean, J. Gary, S. Mizutori, W. Nazarewicz, On the origin of the Wigner energy, Phys. Lett. B 407 103(1997).

[12] R. R. Chasman, n-p pairing-diagonal matrix elements: Wigner energy, symmetry energy and spectroscopy, Phys. Lett. B 577, 47(2003).

[13] P. Ring, Relativistic mean field theory in finite nuclei, Prog. Part. Nucl. Phys. 37, 193(1996)

[14] D. Lunney, International symposium nuclei in the cosmos - V, Volos, Greece, July 6-11, 1998.

[15] Nicola Cabibbo, Unitary symmetry and leptonic decays, Phys. Rev. Lett. 10, 531(1963). 
[16] Makoto Kobayashi, Toshihide Maskawa, CP-violation in the renormalizable theory of weak interaction, Prog. Theor. Phys. 49, 652(1973).

[17] E. Fermi, Versuch einer Theorie der beta-Strahlen. I., Z. Phys. 88, 161(1934).

[18] C. A. Bertulani, H. Schechter, Introduction to nuclear physics, Nova Science Publishers, Inc., New York, 2002.

[19] I. S. Towner, J. C. Hardy, The evaluation of $V_{u d}$, experiment and theory, J. Phys. G: Nucl. Part. Phys. 29, 197(2003).

[20] J. C. Hardy, I. S. Towner, Superallowed $0^{+} \rightarrow 0^{+}$nuclear $\beta$ decays: A critical survey with tests of the conserved vector current hypothesis and the standard model, Phys. Rev. C71, 055501(2005).

[21] I. S. Towner, J. C. Hardy, Calculated corrections to superallowed Fermi $\beta$ decay: New evaluation of the nuclear-structure-dependent terms, Phys. Rev. C66, 035501(2002).

[22] Hartmut Abele, The standard model and the neutron $\beta$-decay, Nucl. Instrum. Methods A 440, 499(2000).

[23] Hendrik Schatz, The importance of nuclear masses in the astrophysical rpprocess, astro-ph/0607625, 2006. 
[24] Karl-Ludwig Kratz, Bernd Pfeiffer, Friedrich-Karl Thielemann and William B. Walters, Nuclear structure studies at ISOLDE and their impact on the astrophysical r-process, Hyperfine Interactions 129, 185(2000).

[25] H. Geissel and G. Münzenberg, Secondary exotic nuclear beams, Annu. Rev. Nucl. Part. Sci. 45, 163(1995).

[26] Jason Allan Clark, Investigating the astrophysical rp-process through atomic mass measurements, PhD thesis, University of Manitoba (2005).

[27] W. Mittig, A. Lépine-Szily, N. A. Orr, Mass measurement far from stability, Annu. Rev. Nucl. Sci. 47 27(1997).

[28] D. R. Goosman and R. W. Kavanagh, ${ }^{10} \operatorname{Be}(d, p){ }^{11} \mathrm{Be}$ and the ${ }^{10} \mathrm{Be}(d, \alpha){ }^{8} \mathrm{Li}$ reactions, Phys. Rev. C 1, 1939(1970).

[29] H. Savajols, The SPEG mass measurement program at GANIL, Hyp. Int. 132, $145(2001)$

[30] T. Radon, H. Geissel, G Münzenberg, B. Franzke, Th. Kerscher, F. Nolden, Yu. N. Novikov, Z. Patyk, C. Scheidenberger, F. Attallah, K. Beckert, T. Beha, F. Bosch, H.Eickhoff, M. Falch, Y. Fujita, M. Hausmann, F. Herfurth, H. Irnich, H. C. Jung, O. Klepper, C. Kozhuharov, Yu. A. Litvinov, K. E. G. Löbner, F. Nickel, H. Reich, W. Schwab, B. Schlitt, M. Steck, K. Sümmerer, T. Winkler, 
H. Wollnik, Schottky mass measurements of stored and cooled neutron-deficient projctile fragments in the element range of $57 \leq Z \leq 84$, Nucl. Phys. A 677, $75(2000)$

[31] B. Schlitt, K. Beckert, T. Beha, H. Eickhoff, B. Franzke, H. Geissel, H. Irnich, H. C. Jung, T. F. Kerscher, O. Klepper, K. E. G. Löbner, G.Münzenberg, F. Nolden, Y. Novikov, T. Radon, H. Reich, A. Schwinn, M. Steck, K. Sümmerer, T. Winkler and H. Wollnik, Schottky mass spectrometry at the heavy ion storage ring ESR, Hyperfine Interactions 99, 117(1996).

[32] M. Hausmann, F. Attallah, K. Beckert, F. Bosch, A. Dolinskiy, H. Eickhoff, M. Falch, B. Franczak, B. Franzke, H. Geissel, Th. Kerscher, O. Klepper, H.J. Kluge, C. Kozhuharov, K. E. G. Löbner, G. Münzenberg, F. Nolden, Yu.N. Novikov, T. Radon, H. Schatz, C. Scheidenberger, J. Stadlmann, M. Steck, T. Winkler, H. Wollnik, First isochronous mass spectrometry at the experimental storage ring ESR, Nucl. Instr. and Meth. A 446 569(2001).

[33] M. D. Lunney, G. Audi, C. Borcea, M. Dedieu, H. Doubre, M. Duma, M. Jacotin, J.-F. Képinski, G. Le Scornet, M. de Saint Simon and C. Thibault, MISTRAL: A new program for precise atomic mass determinations of nuclides far from stability, Hyperfine Interactions 99, 105(1996).

[34] J. Dilling, P. Bricault, M. Smith, H. -J. Kluge and TITAN collaboration, The 
proposed TITAN facility at ISAC for very precise mass measurements on highly charged short-lived isotopes, Nucl. Instrum. Methods B 204, 492(2003).

[35] Klaus Blaum, High-accuracy mass spectrometry with stored ions, Phys. Rep. $425,1(2006)$.

[36] M. König, G. Bollen, H. -J. Kluge, T. Otto, J. Szerypo, Quadrupole excitation of stored ion motion at the true cyclotron frequency, International Journal of Mass Spectrometry and Ion Processes 142, 95(1995).

[37] G. Bollen, S. Becker, H.-J. Kluge, M. Konig, R.B. Moore, T. Otto, H. RaimbaultHartmann, G. Savard, L. Schweikhard, H. Stolzenberg, the ISOLDE Collaboration, ISOLTRAP: a tandem Penning trap system for accurate on-line mass determination of short-lived isotopes, Nucl. Instrum. Methods A 368, 675(2003).

[38] G. Savard, J. Clark, C. Boudreau, F. Buchinger, J. E. Crawford, H. Geissel, J. P. Greene, S. Gulick, A. Heinz, J. K. P. Lee, A. Levand, M. Maier, G. Munzenberg, C. Scheidenberger, D. Seweryniak, K. S. Sharma, G. Sprouse, J. Vaz, J. C. Wang, B. J. Zabransky, Z. Zhou, the S258 Collaboration, Development and operation of gas catchers to thermalize fusion-evaporation and fragmentation products, Nucl. Instrum. Methods A 204, 582(2003).

[39] G. Savard, R. C. Barber, C. Boudreau, F. Buchinger, J. Caggiano, J. Clark, J. E. Crawford, H. Fukutani, S. Gulick, J. C. Hardy, A. Heinz, J. K. P. Lee, R. B. 
Moore, K. S. Sharma, J. Schwartz, D. Seweryniak, G. D. Sprouse and J. Vaz, The Canadian Penning trap spectrometer at Argonne, Hyperfine Interactions $132,223(2001)$.

[40] Tao Sun, High precision mass measurement of ${ }^{37} \mathrm{Ca}$ and developments for LEBIT, $\mathrm{PhD}$ thesis, Michigan State University (2006).

[41] Ryan Ringle, High-precision mass measurement of ${ }^{38} \mathrm{Ca}$ and development of the LEBIT 9.4-T Penning trap system, PhD thesis, Michigan State University (2006).

[42] R. Ringle, P. Schury, T. Sun, G. Bollen, D. Davies, J. Huikari, E. Kwan, Precision mass measurements with LEBIT at MSU, International Journal of Mass Spectrometry 251, 300(2006).

[43] R. Ringle, T. Sun, G. Bollen, D. Davies, M. Facina, J. Huikari, E. Kwan, D. J. Morrissey, A. Prinke, J. Savory, P. Schury, S. Schwarz, and C. S. Sumithrarachchi, High-precision Penning trap mass measurements of ${ }^{37,38} \mathrm{Ca}$ and their contributions to conserved vector current and isobaric mass multiplet equation, Phys. Rev. C 75, 055503(2007).

[44] G. Rouleau, H. Borgenstrand, C. Carlberg, R. Schuch and F. Söderberg, The SMILETRAP (Stockholm-Mainz-Ion-LEvitation-TRAP) facility, Hyperfine Interactions 99 73(1996). 
[45] I. Bergström, C. Carlberg, T. Fritioff, G. Douysset, J. Schönfelder, R. Schuch, SMILETRAP-A Penning trap facility for precision mass measurements using highly charged ions, Nucl. Instrum. Methods A 487, 618(2003).

[46] Guilhem Douysset, Tomas Fritioff, and Conny Carlberg, Determination of the ${ }^{76}$ Ge Double Beta Decay Q Value, Phys. Rev. Lett. 86,4259(2001).

[47] M. Dombsky, P. Bricault, T. Hodges, A. Hurst, P. Schmor, Online isotope separation at ISAC with a $10 \mu \mathrm{A}$ proton driver beam, Nucl. Phys. A 701,486c(2002).

[48] M. Dombsky, P. Bricault, P. Schmor, M. Lane, ISAC target operation with high proton currents, Nucl. Instr. and Meth. B 204 191(2003).

[49] Pierre G. Bricault and Helmut Weick, Use of the Chalk River mass separator for ISAC, Design Note, TRI-DN-97-16, September 1997.

[50] G. Bollen, Mass measurements of short-lived nuclides with ion traps, Nucl. Phys. A $693,3(2001)$.

[51] M. Smith, A square-wave-driven radiofrequency quadrupole cooler and buncher for TITAN, M.Sc. thesis, University of British Columbia (2005).

[52] P. H. Dawson, Quadrupole mass spectrometry and its applications, American Institute of Physics(1995). 
[53] Mathew Smith, Laura Blomeley, Paul Delheij, Jens Dilling, First tests of the TITAN digital RFQ beam cooler and buncher, Trapped charged particles and fundamental physics (TCP06), Parksville, Sept 3-8, TRI-PP-07-26, May 2007.

[54] M. A. Levine, R. E. Marrs, J. R. Henderson, D. A. Knapp, and M. B. Schneider, The electron beam ion trap: a new instrument for atomic physics measurements, Phys. Scr. T22, 157(1988).

[55] David Vogel, Design and operation of the electron beam ion trap, UCRL-I D104990 (National Technical Information Service, Springfield, VA, 1990).

[56] Frederick John Currell, Junji Asada, Koichi Ishii, Arimichi Minoh, Kenji Motohashi, Nobuyuki Nakamura, Kazou Nishizawa, Shunsuke Ohtani, Kiyohiko Okazaki, Makoto Sakurai, Hiroshi Shiraishi, Seiji Tsurubuchi and Hirofumi Watanabe, A new versatile electron-beam ion trap, Journal of the Physical Society of Japan 65, 3186(1996).

[57] Gabriel Herrmann, Optical theory of thermal velocity effects in cylindrical electron beams, J. Appl. Phys. 29, 127(1958).

[58] Reinard Becker, Collision physics in ECR and EBIS/T, Rev. Sci. Instrum. 73, 693(2002).

[59] B. M. Penetrante, J. N. Bardsley, D. DeWitt, M. Clark, and D.Schneider, Evo- 
lution of ion-charge-state distributions in an electron-beam ion trap, Phys. Rev. A $43,4861(1991)$.

[60] Wolfgang Lotz, An empirical formula for the electron-impact ionization crosssection, Z. Phys. 206, 205(1967).

[61] Wolfgang Lotz, Electron-impact ionization cross-sections and ionization rate coeffcients for atoms and ions from hydrogen to calcium, Z. Phys. 216, 241(1968).

[62] A. Müller and E. Salzborn, Scaling of cross sections for multiple electron transfer to highly charged ions colliding with atoms and molecules, Phys. Lett. A 62, $391(1977)$.

[63] N. Selberg, C. Biedermann, and H. Cederquist, Semiempirical scaling laws for electron capture at low energies, Phys. Rev. A 54, 4127(1996).

[64] Michael Froese, The TITAN electron beam ion trap: Assembly, characterization, and first tests, M.Sc. thesis, University of Manitoba (2006)

[65] Lowell S. Brown and Gerald Gabrielse, Geonium theory: Physics of a single electron or ion in a penning trap, Rev. Mod. Phys. 58, 233(1986).

[66] G. Bollen, R. B. Moore, G. Savard and H. Stolzenberg, The accuracy of heavyion mass measurements using time of flight-ion cyclotron resonance in a Penning trap, J. Appl. Phys. 68, 4355(1990). 
[67] A. Dahl David, SIMION 3D version 7.0 users manual, Idaho National Engineering and Environmental Laboratory.

[68] W. M. Itano, J. C. Bergquist, J. J. Bollinger, D. J. Wineland, Cooling methods in ion traps, Physica Scripta T59, 106(1995).

[69] R. C. Thompson, Precision measurement aspects of ion traps, Meas. Sci. Technol. $1,93(1990)$.

[70] Gerald Gabrielse, Detection, damping, and translating the center of the axial oscillation of a charged particle in a Penning trap with hyperbolic electrodes, Phys. Rev. A 29, 462(1984).

[71] Earl C. Beaty, Calculated electrostatic properties of ion traps, Phys. Rev. A 33, $3645(1986)$

[72] H. G. Dehmelt, Radiofrequency spectroscopy of stored ions, Adv. At. Mol. Phys. 5, 109(1969).

[73] R. E. Marrs, Self-cooling of highly charged ions during extraction from electron beam ion sources and traps, Nucl. Instrum. Methods B 149, 182(1999).

[74] Edward A. Mason, Earl W. McDaniel, Transport properties of ions in gases, John Wiley \& Sons, New York, 1988. 
[75] L. A. Viehland, E. A. Mason, W. F. Morrison, M. R. Flannery, Tables of transport collision integrals for $(n, 6,4)$ ion-neutral potential, At. Data Nucl. Data Tables $16,495(1975)$.

[76] F. Herfurth, J. Dilling, A. Kellerbauer, G. Bollen, S. Henry, H.-J. Kluge, E. Lammour, D. Lunney, R. B. Moore, C. Scheidenberger, S. Schwarz, G. Sikler, J. Szerypo, A linear radiofrequency ion trap for accumulation, bunching, and emittance improvement of radioactive ion beams, Nucl. Instrum. Methods A $469,254(2001)$.

[77] A. Nieminen, J. Huikari, A. Jokinen, J. Äystö, P. Campbell, E. C. A. Cochrane, Beam cooler for low-energy radioactive ions, Nucl. Instrum. Methods A 469, $244(2001)$

[78] A. Kellerbauer, T. Kim, R. B. Moore, P. Varfalvy, Buffer gas cooling of ion beams, Nucl. Instrum. Methods A 469, 276(2001).

[79] R. B. Moore, Buffer gas cooling of ion beams, Lecture Notes-Web Page http://www.physics.mcgill.ca/ moore/Notes/BeamCooling.pdf

[80] A. Ashkin, Atomic-beam deflection by resonance-radiation pressure, Phys. Rev. Lett. 25, 1321(1970). 
[81] T. W. Hansch, and A. L. Schawlow, Cooling of gases by laser radiation, Opt. Comm. 13, 68(1975).

[82] D. J. Wineland, Wayne M. Itano, Laser cooling of atoms, Phys. Rev. A 20, 1521(1979).

[83] Stig Stenholm, The semiclassical theory of laser cooling, Rev. Mod. Phys. 58, 699(1986).

[84] L. Spitzer, Physics of fully ionized gases, Interscience Publishers INC., New York 1956.

[85] S. T. Butler and M. J. Buckingham, Energy loss of a fast ion in a plasma, Phys. Rev. 126, 1(1962).

[86] N. E. Frankel, Dominant and non-dominant terms in the energy transfer equations for a fully ionized plasma, plasma physics 7, 225(1965).

[87] N. E. Frankel, K. C. Hines, and R. L. Dewar, Energy loss due to binary collisions in a relativistic plasma, Phys. Rev. A 20, 2120(1979).

[88] Yongbin Chang and C. A. Ordonez, Plasma two-temperature equilibration rate, Phys. Rev. E 69, 03740(2004).

[89] G. Zwicknagel, AIP Conf. Proc. 821: Beam cooling and related topics, p. 513 (2006). 
[90] S. L. Rolston and G. Gabrielse, Cooling antiprotons in an ion trap, Hyperfine Interactions 44, 233(1988).

[91] Takao M. Kojima, Nagayasu Oshima, Dana Dumitriu, Hitoshi Oyama, Akihiro Mohri, and Yasunori Yamazaki, An ion trap for cooling MCIs with cold positrons, RIKEN Review 31, 70(2000).

[92] J. Bernard, J. Alonso, T. Beier, M. Block, S. Djekic, H,-J. Kluge, C. Kozhuharov, W. Quint, S. Stahl, T. Valenzuela, J. Verdú, M. Vogel, G. Werth, Electron and positron cooling of highly charged ions in a cooler Penning trap, Nucl. Instrum. Methods A 532, 224(2004).

[93] Yukap Hahn, Electron-ion recombination processes-an overview, Rep. Prog. Phys. 60, 691(1997).

[94] Alfred Müller, Andreas Wolf, Production of antihydrogen by recombination of $\bar{p}$ with $e^{+}$: What can we learn from electron-ion collision studies? Hyperfine Interactions 109, 233(1997).

[95] G. Kilgus, D. Habs, D. Schwalm and A. Wolf, High-resolution measurement of dielectronic recombination of lithiumlike $\mathrm{Cu}^{26+}$, Phys. Rev. A 46, 5730(1992).

[96] D. A. Knapp, R. E. Marrs, M. B. Schneider, M. H. Chen, M. A. Levine and P. Lee, Dielectronic recombination of heliumlike ions, Phys. Rev. 47, 2039(1993). 
[97] D. Nikolić, E. Lindroth, S. Kieslich, C.Brandau, S. Schippers, W. Shi, A. Müller, G. Gwinner, M. Schnell and A. Wolf, Dielectronic recombination resonances in $\mathrm{Na}^{8+}$, Phys. Rev. A 70, 062723(2004).

[98] M. Pajek, R. Schuch, Three-body recombination of ions with electrons in cooler storage rings, Hyperfine interactions 108, 185(1997).

[99] M. Pajek and R. Schuch, Radiative recombination of bare ions with low-energy free electrons, Phys. Rev. A 45, 7894(1992).

[100] R. Schuch, A. Bárány, H. Danared, N. Elander, and S. Mannervik, Storage rings, a new tool for atomic physics, Nucl. Instrum. Methods B 43, 411(1989).

[101] D. A. Verner and G. J. Ferland, Atomic data for astrophysics. I. Radiative recombination rates for H-like, He-like, Li-like and Na-like ions over a broad range of temperature, ApJS 103, 467(1996).

[102] M. F. Gu, Radiative recombination rate coefficients for bare through F-like isosequences of $\mathrm{Mg}, \mathrm{Si}, \mathrm{S}, \mathrm{Ar}, \mathrm{Ca}, \mathrm{Fe}$, and Ni, Astrophys. J. 589, 1085(2003).

[103] N. R. Badnell, Radiative recombination data for modelling dynamic finitedensity plasmas, astro-ph/0604144, 2006.

[104] P. Mazzotta, G. Mazzitelli, S. Colafrancesco, and N. Vittorio, Ionization balance 
for optically thin plasmas: Rate coefficients for all atoms and ions of the elements H to Ni, Astron. Astrophys. Suppl. Ser. 133, 403(1998).

[105] J. Danielson and C. Surko, Torque-balanced high-density steady states of singlecomponent plasmas, Phys. Rev. Lett. 94, 035001(2005)

[106] G. Gabrielse, X. Fei, L. A. Orozco, R. L. Tjoelker, J. Haas, H. Kalinowsky, T. A. Trainor, and W. Kells, Cooling and slowing of trapped antiprotons below 100 meV, Phys. Rev. Lett. 63, 1360(1989).

[107] Nagayasu Oshima, Megumi Niigaki, Masato Inoue, Takao M. Kojima, Akihiro Mohri, Yasuyuki Kanai, Yoichi Nakai, Ken-ichiro Komaki and Yasunori Yamazaki, Project to produce cold highly charged ions using positron and electron cooling techniques, J. Phys.: Conf. Ser. 2 127(2004).

[108] Th. Beier, L. Dahl, H.-J. Kluge, Chr. Kozhuharov, and W. Quint, HITRAP technical design report.

[109] Xiang Fei, W. M. Snow, Cylindrical Penning traps with dynamic orthogonalized anharmonicity compensation for precision experiments, Nucl. Instrum. Methods A $425,431(1999)$.

[110] William H. Press, Brian P. Flannery, Saul A. Teukolsky, William T. Vettering, Numerical recipes in C, Cambridge, UK: Cambridge University Press, 1988. 
[111] Isao Tanihata, Neutron halo nuclei, J. Phys. G: Nucl. Part. Phys. 22, 157(1996).

[112] I. Tanihata, H. Hamagaki, O. Hashimoto, Y. Shida, N. Yoshikawa, K. Sugimoto, O. Yamakawa, T. Kobayashi, and N. Takahashi, Measurements of interaction cross sections and nuclear radii in the light p-shell region, Phys. Rev. Lett. 55, $2676(1985)$

[113] G. Audi, A. H. Wapstra and C. Thibault, The AME2003 atomic mass evaluation (II). Tables, graphs and references, Nucl. Phys. A 729, 337(2003).

[114] A. H. Wapstra, G. Audi, C. Thibault, The AME2003 atomic mass evaluation (I). Evaluation of input data, adjustment procedures, Nucl. Phys. A729 129(2003).

[115] A. Kellerbauer, K. Blaum, G. Bollen, F. Herfurth, H. -J. Kluge, M. Kuckein, E. Sauvan, C. Scheidenberger, and L. Schweikhard, From direct to absolute mass measurements: A study of the accuracy of ISOLTRAP, Eur. Phys. J. D 22, $53(2003)$

[116] Mathew Jonathon Smith, A mass measurement of the short-lived halo nucleus ${ }^{11} \mathrm{Li}$ with the TITAN Penning trap spectrometer, $\mathrm{PhD}$ thesis, University of British Columbia (2007).

[117] Donald A. Gurnett and Amitava Bhattacharjee, Introduction to plasma physics, Cambrige, UK: Cambridge University Press, 2005. 
[118] Paul W. Allison, Joseph D. Sherman, and David B. Holtkamp, An emittance scanner for intense low-energy ion beams, IEEE Transactions on Nuclear Science, Vol. NS-30,2204(1983). 